\title{
Phase equilibrium and cage occupancy calculations of carbon dioxide hydrates using ab initio intermolecular potentials
}

\author{
Srinath Chowdary Velaga \\ West Virginia University
}

Follow this and additional works at: https://researchrepository.wvu.edu/etd

\footnotetext{
Recommended Citation

Velaga, Srinath Chowdary, "Phase equilibrium and cage occupancy calculations of carbon dioxide hydrates using ab initio intermolecular potentials" (2009). Graduate Theses, Dissertations, and Problem Reports. 2055.

https://researchrepository.wvu.edu/etd/2055

This Thesis is protected by copyright and/or related rights. It has been brought to you by the The Research Repository @ WVU with permission from the rights-holder(s). You are free to use this Thesis in any way that is permitted by the copyright and related rights legislation that applies to your use. For other uses you must obtain permission from the rights-holder(s) directly, unless additional rights are indicated by a Creative Commons license in the record and/ or on the work itself. This Thesis has been accepted for inclusion in WVU Graduate Theses, Dissertations, and Problem Reports collection by an authorized administrator of The Research Repository @ WVU. For more information, please contact researchrepository@mail.wvu.edu.
} 


\title{
Phase Equilibrium and Cage Occupancy Calculations of Carbon Dioxide Hydrates using $A b$ Initio Intermolecular Potentials
}

\author{
Srinath Chowdary Velaga
}

Thesis submitted to

College of Engineering and Mineral Resources

at West Virginia University

in partial fulfillment of the requirements

for the degree of

\author{
Master of Science \\ in \\ Chemical Engineering
}

Dr. Brian J. Anderson

Dr. Alfred Stiller

Dr. Wu Zhang

Department of Chemical Engineering

Morgantown, West Virginia

2009

Key words: Gas hydrates, $\mathrm{CO}_{2}$ hydrates, Intermolecular potentials, ab initio calculations, Phase equilibrium, Cage occupancy, Cell potentials. 


\title{
Phase Equilibrium and Cage Occupancy Calculations of Carbon Dioxide Hydrates using $A b$ Initio Intermolecular Potentials
}

\author{
Abstract \\ Srinath Chowdary Velaga
}

Huge deposits of carbon is trapped in the form of methane gas hydrates, these methane gas hydrates represent a potential energy source that could possibly last for thousands of years. Gas hydrate reservoirs are receiving increased attention as potential locations for $\mathrm{CO}_{2}$ sequestration, with $\mathrm{CO}_{2}$ replacing the methane that is recovered as an energy source.

In this scenario it is very important to correctly characterize the cage occupancies of $\mathrm{CO}_{2}$ to correctly assess the sequestration potential as well as the methane recoverability. In order to predict accurate cage occupancies, the guest-host interaction potential must be represented properly. Earlier, these potential parameters were obtained by fitting to experimental data and these fitted parameters do not match with those obtained by second virial coefficient or gas viscosity data. Ab initio quantum mechanical calculations provide an independent means to directly obtain accurate intermolecular potentials. A potential energy surface (PES) between $\mathrm{H}_{2} \mathrm{O}$ and $\mathrm{CO}_{2}$ was computed at the MP2/aug-cc-pVTZ level and corrected for basis set superposition error (BSSE), an error caused due to the lower basis set, by using 0.361 of the full counterpoise and 0.639 of the uncorrected energy correction. Intermolecular potentials were obtained by fitting Exponential-6 and Lennard-Jones 6-12 models to the $a b$ initio PES, correcting for many-body interactions. Reference parameters for structure I carbon dioxide hydrate has been calculate with this site-site ab initio intermolecular potentials as $\Delta \mu_{w}{ }^{\circ}=1204 \pm 3 \mathrm{~J} / \mathrm{mol}$ and $\Delta H_{w}{ }^{\circ}=1189 \pm 12 \mathrm{~J} / \mathrm{mol}$. The pure $\mathrm{CO}_{2}$ hydrate equilibrium pressure was predicted with an average absolute deviation of less than $2 \%$ from the experimental data. Predictions of the small cage occupancy ranged from 22-38\% and the hydration number for the $\mathrm{CO}_{2}$ hydrate was calculated to be above 7.0, whereas the large cage is more than $98 \%$ occupied.

Cell potential parameters, the potential well depths and volumes of negative energy, have been found for carbon dioxide hydrate system from the center-well solution. The Langmuir constants are computed from the $a b$ initio site-site intermolecular potentials. These Cell potential parameters can be used to predict the mixed hydrate properties for carbon dioxide with other guest molecule. 


\section{Acknowledgements}

I express my gratitude to my advisor Dr. Brian J. Anderson for giving me the opportunity to pursue this research and guiding me throughout this work. With his enthusiasm, his inspiration, and his great efforts to explain things clearly and simply, he made research as fun for me. Working with him is an invaluable experience.

I would like to express my deep appreciation to my committee members Dr. Alfred Stiller and Dr. Wu Zhang for being on my thesis committee and providing me with invaluable comments and advice on my thesis.

I would like to thank my father, Bhavani Prasad, my mother, Vidhyadari, and my brother, Srikanth Chowdary, for their inseparable support and prayers and their love, affection and encouragement in all the phases of my life. Without your unending support and love from childhood to now, I would never have made it through this process or any of the tough times in my life.

My special thanks to Dr. Suman Thotla, who encouraged me to go to graduate school. Finally, I would like to thank my roommates, lab mates and all other friends for their support, love and encouragement. Thank you. 


\section{Preface}

Huge deposits of hydrates are found in permafrost and in continental margins. These gas hydrates, a potential energy source, can also be a possible solution to the carbon dioxide problem. Carbon dioxide could potentially be sequestrated in the form of carbon dioxide hydrates in the ocean sediments below the seafloor in stable geologic strata. It is proposed that carbon dioxide gas can replace the methane in naturally-occurring gas hydrate reservoirs. In order to understand this swapping process and the stability of carbon dioxide sequestration on the ocean floor, the accuracy of the thermodynamic model of gas hydrates is very important. One very important term in the thermodynamic model is the intermolecular potential between the guest and the host water molecules. In previous work, these potential parameters were obtained by fitting to monovariant experimental data, resulting in fitted parameters that do not match those obtained by second virial coefficient or gas viscosity data.

In Chapter 1, a brief introduction of gas hydrates, natural occurrences, beneficial uses and the crystal structures of hydrates are discussed, including an overview of previous theoretical work on gas hydrates; i.e., intermolecular potentials, phase Equilibria, and cage occupancy. The statistical thermodynamics model, the van der Waals and Platteeuw model, which is used in this study, is discussed in Chapter 2. In this model, the chemical potential of water in the hydrate phase is calculated using a Langmuir adsorption model. This Langmuir constant is important as it is a key term to predict the cage occupancies and phase equilibrium of gas hydrate. The Langmuir constant is the six dimensional configurational integral of the guest molecule and the host water molecules divided by $k T$. In Chapter 2, various methods to evaluate the configurational integral are discussed and the most accurate is found to be the 10point Gauss-Legendre quadrature formula. Various intermolecular potential functions that describe the guest-host interactions are also discussed in this chapter.

To overcome the unphysical nature of intermolecular interaction potentials fit to equilibrium data and their inability to predict the $\mathrm{CO}_{2}-\mathrm{CH}_{4}$ mixed hydrate thermodynamics well, potentials in this work are obtained by an independent ab initio method. In Chapter 3, the $a b$ initio method and the optimum basis set to calculate the potential energy surface is discussed. Site-site intermolecular potentials were obtained by fitting Exponential-6 and Lennard-Jones 6-12 models to a 6,000-point ab initio potential energy surface, correcting for many-body interactions. Reference parameters for structure I carbon dioxide hydrate were calculated using this site-site $a b$ initio intermolecular potential to be $\Delta \mu_{w}{ }^{o}=1204 \pm 3 \mathrm{~J} / \mathrm{mol}$ and $\Delta H_{w}{ }^{o}=1189 \pm 12 \mathrm{~J} / \mathrm{mol}$. With these accurate $a b$ initio intermolecular potentials and reference parameters for carbon dioxide, hydrate cage occupancies and hydrate equilibrium pressure was predicted. 
In Chapter 4, the application of Cell potential method to calculate the phase equilibrium of multi component system has been discussed. The Cell potential parameters are calculated for $\mathrm{CO}_{2}$ hydrate from the ab initio Langmuir constants. 


\section{Table of Contents}

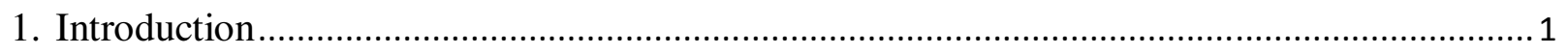

1.1 Overview and History of Gas Hydrates............................................................... 1

1.1.1 Occurrence of Gas Hydrates .....................................................................2

1.1.2 Beneficial uses of hydrates ..................................................................

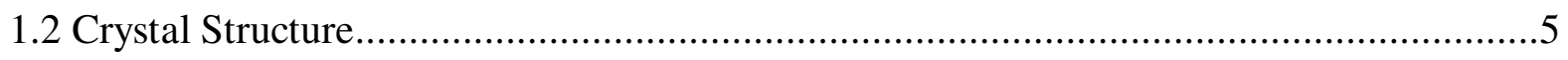

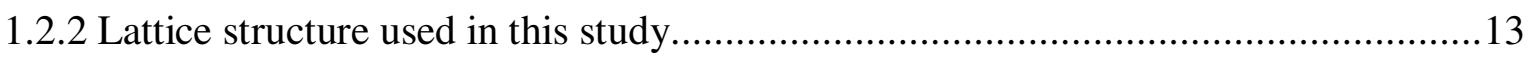

1.2.3 Proton Placement ................................................................................. 13

1.3 Overview of Previous Theoretical work ................................................. 14

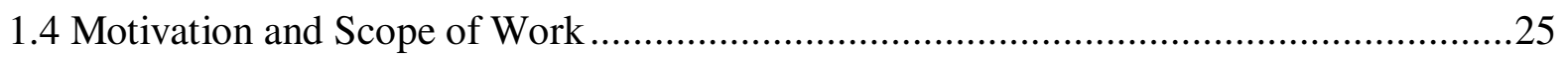

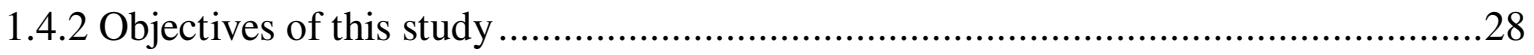

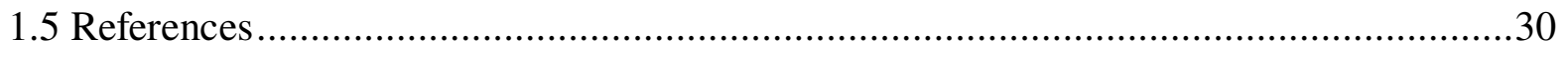

2. Theoretical Model for Gas Hydrates .......................................................................... 33

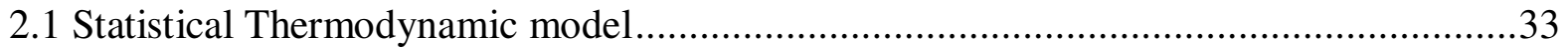

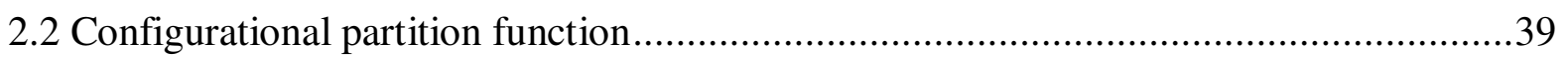

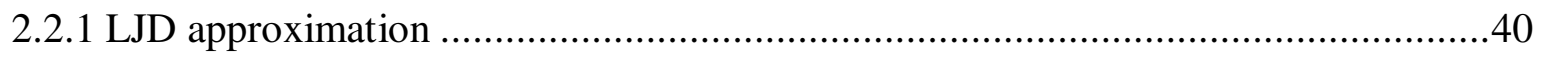

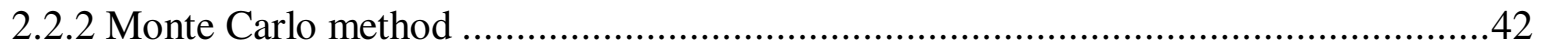

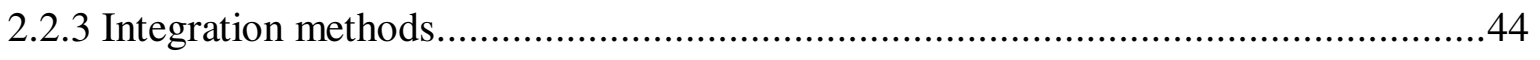

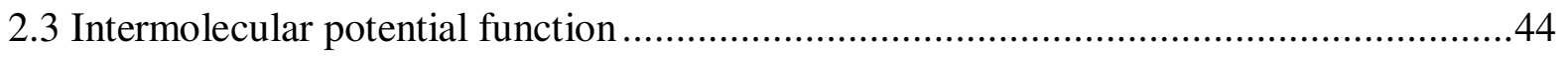

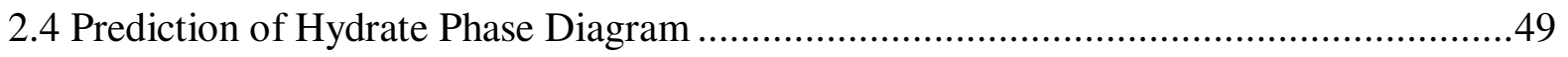

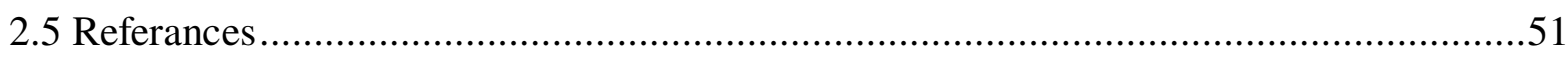

3. Ab Initio Intermolecular Potentials for Predicting Cage Occupancy and Phase Equilibrium

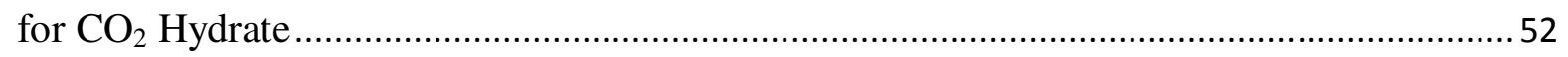

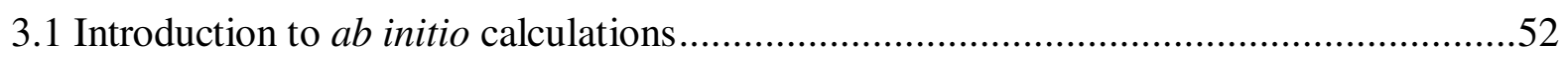

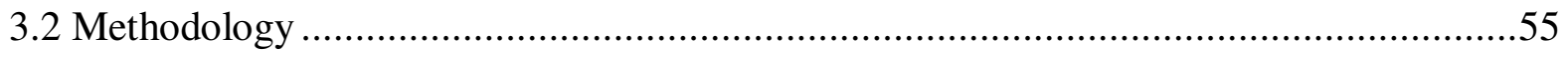

3.2.1 Optimum method for PES calculation .....................................................56

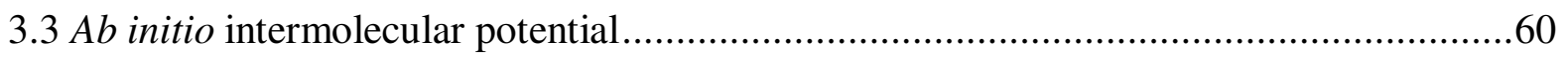

3.3.1 Determination of potential energy surface ............................................60

3.3.2 Potential fit to intermolecular energies .................................................6 66

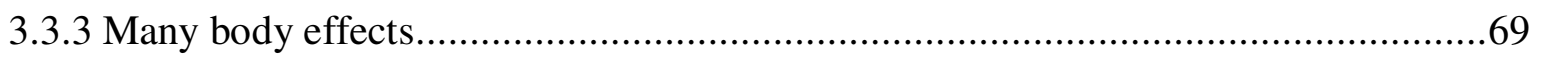




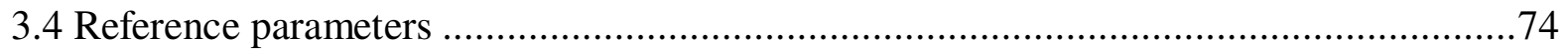

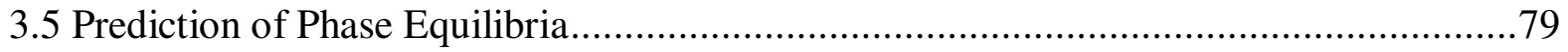

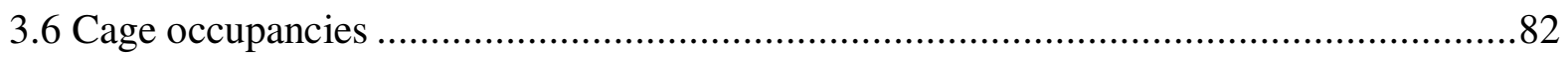

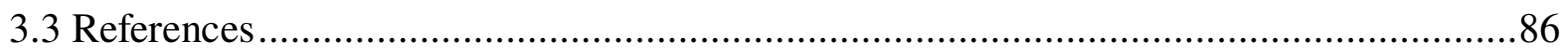

4. Application of cell potential method to calculate the phase equilibrium of multi-component

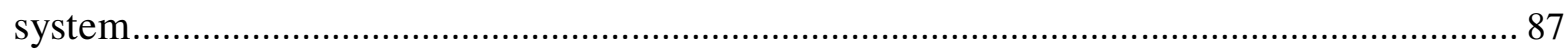

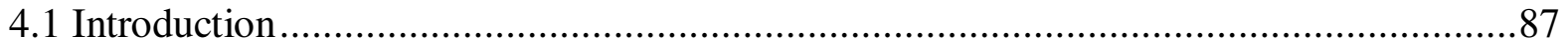

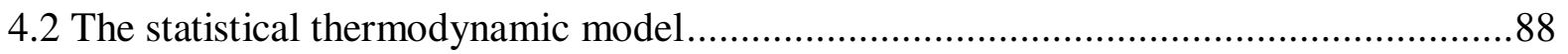

4.3 Configurational Integral Calculation ................................................................91

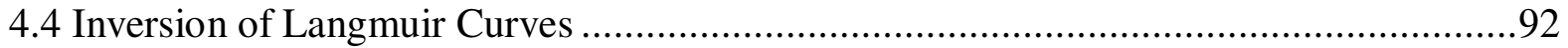

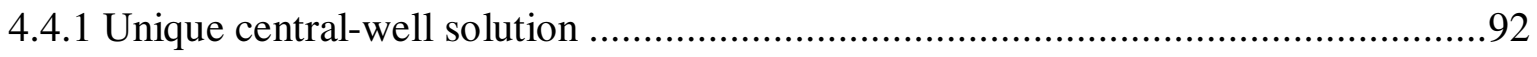

4.4.2 Calculation of Langmuir constant...............................................................94

4.5 Computing Cell Potentials .....................................................................................96

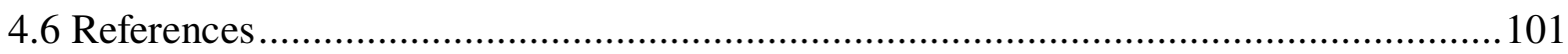

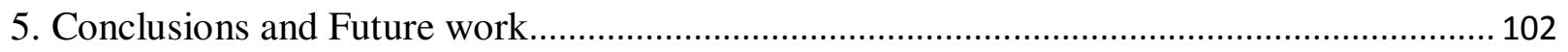

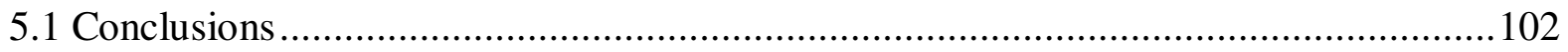

5.2 Recommendations and Future work............................................................. 104 


\section{List of Figures}

Figure1.1 Schematic diagram of $\mathrm{CH}_{4}-\mathrm{C}_{2} \mathrm{H}_{6}$ mixed hydrate replaced with $\mathrm{CO}_{2}$. .................4

Figure1.2 Monovariant phase equilibrium for $\mathrm{CH}_{4}$ and $\mathrm{CO}_{2}$ hydrates..................................5

Figure 1.3 Cavities of Structure 1: (a) pentagonal dodechaderon (small cage, $5^{12}$ ) (b) tetrakaidecahedran (large cage, $5^{12} 6^{2}$ ). .8

Figure1.4 Cavities of Structure II: (a) pentagonal dodechaderon (small cage, $5^{12}$ ) (b) hexakaidecahedron (large cage, $5^{12} 6^{4}$ ).

Figure 1.5 Cavities of Structure H: (a) pentagonal dodechaderon (small cage, $5^{12}$ ) (b) irregular dodechaderon (medium cage, $4^{3} 5^{6} 6^{3}$ )

(c) icosahedron (large cage, $5^{12} 6^{8}$ ). 9

Figure1.6 Lattice structure of Structure I hydrate. ..................................................... 10

Figure1.7 Lattice structure of Structure II hydrate ......................................................... 11

Figure1.8 Lattice structure of Structure $\mathrm{H}$ hydrate ........................................................ 12

Figure1.9 T-shaped structure of $\mathrm{CO}_{2}-\mathrm{H}_{2} \mathrm{O}$ complex.....................................................23

Figure 2.1 Lennard - Jones 6-12 potential parameter .................................................45

Figure 2.2 Kihara intermolecular potential ......................................................... 46

Figure 2.3 Exponential-6 intermolecular potential........................................................48

Figure 2.4 Schematic of computer program for calculating equilibrium pressure...................50

Figure 3.1 Effect of increasing basis set size on the BSSE...........................................59

Figure 3.2 Calculation time and binding energy at each basis set for the $\mathrm{CO}_{2}-\mathrm{H}_{2} \mathrm{O}$ complex...59

Figure 3.3 Planar Orientation of water molecule (a) water plane parallel to the page, plane-1 (b) water plane perpendicular to the page, plane-2 ...........................................................62

Figure 3.4 Six-dimensional orientation of carbon dioxide and water complex. ....................63

Figure 3.5 Parity plot of corrected energies of $\mathrm{CO}_{2}-\mathrm{H}_{2} \mathrm{O}$ calculated at aug-cc-pVTZ basis level w.r.t energies calculated at half counterpoise aug-cc-pV5Z basis level..................66

Figure 3.6 TIP4P water model ............................................................................6

Figure 3.7 Parity plot for water plane-1 showing the number of binding energy points...........69

Figure 3.8 Parity plot for water plane-2 showing the number of binding energy points...........70

Figure 3.9 Single guest $\mathrm{CO}_{2}$ and 15 water molecules of the pentagonal dodecahedron of the structure I hydrate.................................................................................. 73

Figure 3.10 Parity plot of corrected site-site predicted 15 water molecule-carbon dioxide interaction energies. ............................................................................... 73

Figure 3.11 Thermodynamic reference parameters for structure $\mathrm{I} \mathrm{CO}_{2}$ hydrate.....................77

Figure 3.12 Algorithm to calculate the phase equilibrium and cage occupancy......................80

Figure 3.13 Calculation of $\mathrm{CO}_{2}$ hydrate equilibrium dissociation pressure using ab initio sitesite potentials and regressed reference parameters for $\mathrm{CO}_{2} \ldots \ldots \ldots \ldots \ldots \ldots \ldots \ldots \ldots 1$

Figure 3.14 Calculation of $\mathrm{CO}_{2}$ hydrate equilibrium dissociation pressure for $T>260 \mathrm{~K}$, using $a b$ initio site-site potentials and regressed reference parameters for $\mathrm{CO}_{2} \ldots \ldots \ldots \ldots . . .81$

Figure 3.15 Cage occupancy of carbon dioxide hydrate at temperature ranging from $155 \mathrm{~K}$ to $283 \mathrm{~K}$. 
Figure 3.16 Hydration number for carbon dioxide hydrate at different temperature .85

Figure 4.1 van't Hoff behavior indicating the temperature dependency of Langmuir

Figure 4.2 Cell potentials of carbon dioxide in small cage structure I hydrate calculated using

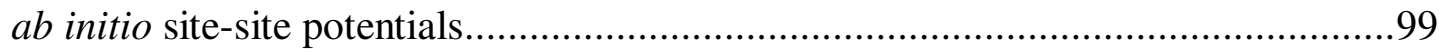

Figure 4.3 Cell potentials of carbon dioxide in large cage structure I hydrate calculated using ab initio site-site potentials ............................................................................ 100 


\section{List of Tables}

Table 1.1 Hydrate crystal structure ....................................................................

Table 2.1 Thermodynamics reference properties for structure I .......................................38

Table 2.2 Thermodynamic reference properties for structure I, $T_{o}=273.15 \mathrm{~K} \ldots \ldots \ldots \ldots \ldots \ldots . . . . . .39$

Table $3.1 \mathrm{CO}_{2}-\mathrm{H}_{2} \mathrm{O}$ binding energies $(\mathrm{kcal} / \mathrm{mol})$ at various levels of theory and basis sets.......57

Table 3.2 Binding energies calculated on $\mathrm{CO}_{2}-\mathrm{H}_{2} \mathrm{O}$ complex with geometry optimized at the

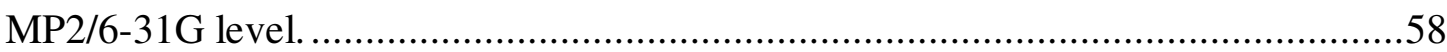

Table 3.3 The binding energies at aug-cc-pV5Z and aug-cc-pVTZ basis level ....................64

Table $3.4 \mathrm{CO}_{2}-\mathrm{H}_{2} \mathrm{O}$ potential parameters by site-site model.........................................72

Table 35 Heat capacity and volumetric reference properties between the empty hydrate lattice and fluid phase (liquid water or ice) .............................................................. 76

Table 4.1 Cell potential parameters for structure I carbon dioxide hydrates ........................97

Table 4.2 Cell potential parameters for structure II (unstable) carbon dioxide hydrate ............97 Table 4.3 Cell potential parameters for structure I hydrate using other intermolecular potentials 


\section{Introduction}

\subsection{Overview and History of Gas Hydrates}

Gas hydrates, also known as gas clathrates, are class of solids in which low molecular weight gas molecules $\left(\mathrm{O}_{2}, \mathrm{H}_{2}, \mathrm{~N}_{2}, \mathrm{CO}_{2}, \mathrm{CH}_{4}, \mathrm{H}_{2} \mathrm{~S}\right.$, Ar, $\mathrm{Kr}$, and $\left.\mathrm{Xe}\right)$ occupy cages made of hydrogen-bonded water molecules. The presence of the guest molecule thermodynamically stabilizes the structure. The term clathrate was first used by Powell ${ }^{1}$ after the Latin word clathrates meaning to be enclosed or protected by cross bars of a grating. In 1811, Sir Humphrey Davy discovered the first gas hydrates ${ }^{2}$; he observed a yellow precipitate while passing chlorine gas through water at temperature near $0^{\circ} \mathrm{C}$ and identified the solid as chlorine hydrate. In addition, there was some evidence that hydrates were retrieved prior to Davy by Joseph Priestley ${ }^{3}$ in 1778 . Priestley observed that the vitriolic air $\left(\mathrm{SO}_{2}\right)$ would impregnate water and cause it to freeze and refreeze to form $\mathrm{SO}_{2}$ hydrate. Wroblewski ${ }^{4,5}$ might be the first to record the evidence of the existence of $\mathrm{CO}_{2}$ hydrate during his studies on carbonic acid. $\mathrm{He}$ observed a white material resembling snow, gas hydrate, formed by raising the pressure above certain limit in his $\mathrm{CO}_{2}-\mathrm{H}_{2} \mathrm{O}$ system.

During first hundred years after Davy's discovery of gas hydrates, the studies on gas hydrates were of academic, concerned with the identification of species that form hydrates and the pressure-temperature conditions at which this formation occurs. In 1934, Hammerschmidt ${ }^{6}$ indicated that the plugging of natural gas pipeline was not due to the formation of ice but due to the formation of clathrate hydrates of natural gas. Considering the significant economic risks in the gas and oil industry, where the oil and gas industry was growing rapidly, a great deal of research has been conducted by the petroleum industry in order to inhibit this phenomenon. It 
marked the beginning of the intense research on natural gas hydrates by the oil and gas industry, government and academia. Since the mid 1960's, with the discovery of the natural gas hydrates, the hydrate research has been motivated by production, transport and processing problems in unusual environments such as North Slope of Alaska, in Siberia and in deep ocean drilling.

\subsubsection{Occurrence of Gas Hydrates}

Naturally on Earth gas hydrates can be found on the seafloor, in ocean sediments, in deep lake sediments, as well as in the permafrost regions. Huge deposits of carbon $\left(2 \times 10^{16}\right.$ $\mathrm{kg}$ ) are trapped in oceanic sediments in the form of methane hydrates ${ }^{7}$. Natural deposits of methane gas hydrates were first discovered in the Soviet Union in the early 1960's and later in many marine types of sediment and in Alaskan permafrost ${ }^{8}$. These hydrates represent a potential energy source that could possibly last for thousands of years. However, estimate of the amount of hydrates decreases as man learns more about hydrates in the environment. The initial global hydrate reserve estimation was given by Trofimuk ${ }^{9}$ with an estimate of $3053 \times$ $10^{15} \mathrm{~m}^{3}$ of methane assuming hydrates could occur wherever sufficiently low temperatures and high pressures exist. Soloview ${ }^{10}$ considered the limiting factors like availability of methane, limited porosity, percentages of organic matter and so on in estimating the hydrate reserve and gave the minimum of all the researches with an estimate of $0.2 \times 10^{15} \mathrm{~m}^{3}$ methane. Klauda and Sandler $^{11}$ presented an equilibrium thermodynamic model for in-place hydrate formation, a different method of estimating hydrates reserves from those of all preceding estimates. They generated a new ab initio thermodynamic model which includes the effect of water salinity, confinement of hydrate in pores and the distribution of pores in the natural sediments to predict 
the hydrate stability in the sea floor. Using this model and a mass transfer description of hydrate formation they predicted the occurrences of methane hydrates. They estimated a total volume of $120 \times 10^{15} \mathrm{~m}^{3}$ of methane gas but this estimates includes very deep hydrates and dispersed small concentrations of hydrates that may dissociates during recovery. When only continental margins are considered they estimated to $44 \times 10^{15} \mathrm{~m}^{3}$ of methane gas expanded to standard temperature and pressure. The energy consumption of the United States for 1000 years at current rate is $1 \times 10^{15} \mathrm{~m}^{3}$. Therefore, the resource of hydrates has a potential of providing the clean energy source for up to 10,000 years $^{12}$. Destabilized methane hydrates may have some effect on the global climate change, methane has green house gas properties, but this effect will probably be minimal at least during the next 100 years $^{7}$.

\subsubsection{Beneficial uses of hydrates}

Hydrates have also been considered as a possible solution to the $\mathrm{CO}_{2}$ problem. The idea of sequestrating the carbon dioxide on the ocean floor to hold the increase in green house gas in the atmosphere has been proposed. Liquid $\mathrm{CO}_{2}$ is injected in to the deep regions of the ocean at depths greater than 1000 meters to form solid clathrates. It is also proposed that the $\mathrm{CO}_{2}$ can be stored in linkage with methane exploitation, as the hydrate formation and dissociation conditions of $\mathrm{CO}_{2}$ and methane hydrates are different. The thermodynamic phase diagram for carbon dioxide and methane are shown in Figure 1.1. This swapping process will help in the sequestering the $\mathrm{CO}_{2}$ and also the source for methane. A microscopic analysis was conducted

by Park et al. ${ }^{13}$ to examine the swapping of $\mathrm{CO}_{2}$ and methane hydrate: for structure ${\mathrm{I} \mathrm{CH}_{4}}_{4}$ hydrate, the $\mathrm{CO}_{2}$ molecules preferably occupy the large cages recovering $64 \%$ of the methane 
and for structure II $\mathrm{CH}_{4}$ hydrate (mixed hydrate with ethane), a structural transition from structure II to structure I and a lattice dimension change occurs. Schematic diagram of $\mathrm{CH}_{4}$ $\mathrm{C}_{2} \mathrm{H}_{6}$ mixed hydrate replaced with $\mathrm{CO}_{2}$ is shown in Figure 1.1. They showed that the recovery of methane gas increased to $84 \%$ when nitrogen is added with $\mathrm{CO}_{2}$ gas. Gas hydrates have been proposed and used in a number of separation processes. They have been used successfully in the desalination of seawater ${ }^{14}$ and in the separation of light gases. Hydrates also have the potential to separate the $\mathrm{CO}_{2}$ gas from the flue gases exhausted by the large power plants ${ }^{15}$. The transportation and storage of natural gas in the form of solid gas hydrates has also been suggested $^{16}$. Hydrate storage of gases has benefits of lower storage space and low pressures for safety. Finally, the use of their dissociation energy can be applied in a refrigeration process or cool storage.

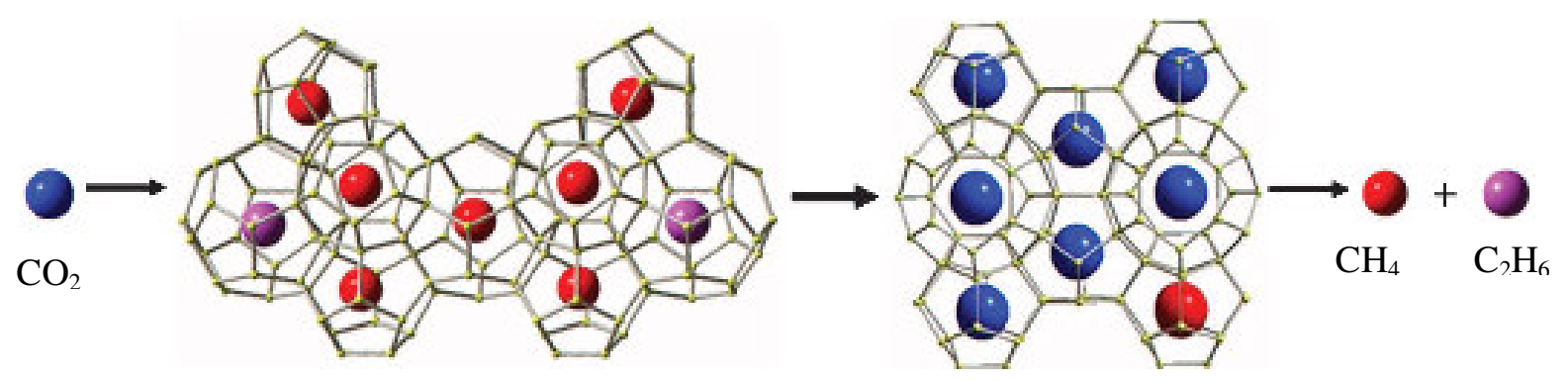

Figure1.1 Schematic diagram of $\mathrm{CH}_{4}-\mathrm{C}_{2} \mathrm{H}_{6}$ mixed hydrate replaced with $\mathrm{CO}_{2}{ }^{13}$. 


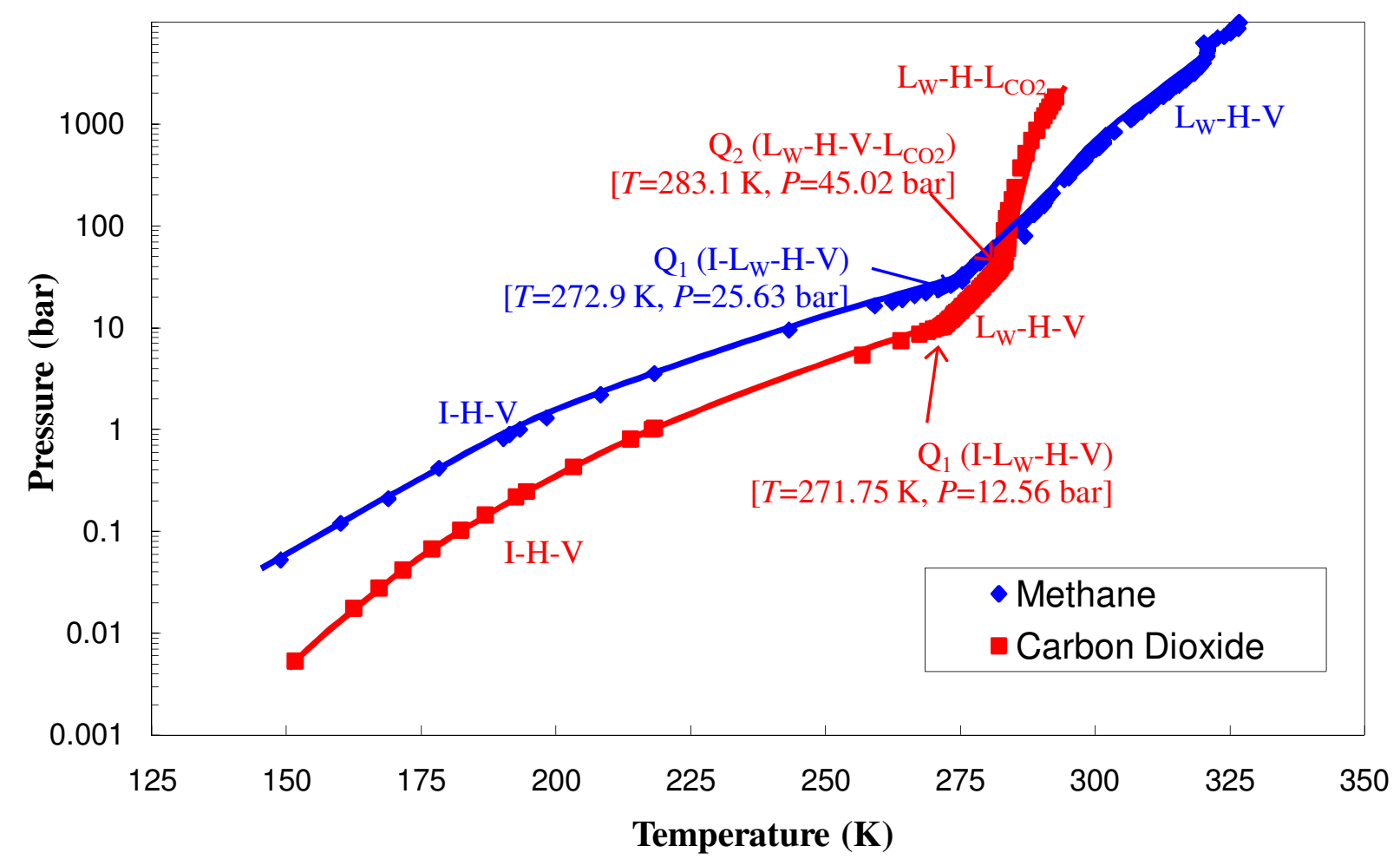

Figure1.2 Monovariant phase equilibrium for $\mathrm{CH}_{4}$ and $\mathrm{CO}_{2}$ hydrates

\subsection{Crystal Structure}

Hydrates are formed due to the unusual behavior of the $\mathrm{H}_{2} \mathrm{O}$ molecules. In ice, water molecules are arranged in hexagonal form. Each water molecule is attached by four neighboring water molecules through hydrogen bonding. The oxygen atoms of the $\mathrm{H}_{2} \mathrm{O}$ molecules are tetrahedrally coordinated in the clathrates hydrate but not as regular as in the ice. This deviation from regularity is due to the polyhedra (a combination of hexagonal, pentagonal and square faces) formed from hydrogen bonded water molecules. The combination of these basic cavities forms different hydrate structures ${ }^{17}$. Clathrate hydrate can possess many different 
crystal structures ${ }^{18}$, but only three structures are known to occur in natural environments: structure I (sI), structure II (sII) and structure $\mathrm{H}(\mathrm{sH})$. The nomenclature suggested by Jeffry and McMullan ${ }^{19}$ for basic cavities of hydrate structures is $n^{m}$, where $n$ is the number of edges and $m$ is the number of faces.

In structure I, each unit cell has 2 small and 6 large cavities. The small cavity is composed of 20 water molecules arranged to form 12 pentagonal faces $\left(5^{12}\right)$ and the resulting polyhedra is known as pentagonal dodecahedra. The large cavity contains 24 water molecules, which form 12 pentagonal and 2 hexagonal faces $\left(5^{12} 6^{2}\right)$ and the polyhedra is tetrakaidecahedra. Structure I has total of 46 water molecules per unit cell and form the primitive cubic lattice with lattice constant of $12.0 \AA$. The cavities of the Structure I are shown in the Figure 1.2. The ideal structural composition for a fully occupied structure I is $8 \mathrm{X} \cdot 46 \mathrm{H}_{2} \mathrm{O}$ where $\mathrm{X}$ is the guest molecule.

Structure II has sixteen $5^{12}$ cavities and eight $5^{12} 6^{4}$ (hexakaidecahedra), which is a 16sided cage, per unit cell. It has total of 136 water molecule per unit cell and form the face centre cubic lattice with lattice constant of $17.3 \AA^{20}$. The cavities of the structure II are shown in the Figure 1.3. The ideal structural composition for a fully occupied structure I is $24 \mathrm{X} .136 \mathrm{H}_{2} \mathrm{O}$ where $\mathrm{X}$ is the guest molecule. Structure $\mathrm{H}$ hydrate was reported by Ripmeester et al. ${ }^{21}$ and the unit cell has 34 molecules with the composition: 3 cages of $5^{12}, 2$ cages of $4^{3} 5^{6} 6^{3}$ (irregular dodecahedron,) and 1 cage of $5^{12} 6^{8}$ (icosahedrons). The cavities of structure $\mathrm{H}$ are shown in Figure 1.4. Unlike sI and sII, which generally forms hydrate with single occupant either the small or large cavity the structure $\mathrm{H}$ requires two sizes of molecules to stabilize the structure. The properties of the structures are tabulated in Table 1. The lattice structure of structure I, structure II and structure $\mathrm{H}$ are shown in Figure 1.5, Figure 1.6 and Figure 1.7 respectively. 
The presence of the guest molecule stabilizes the host lattice structure because of the relatively weak van der Waals interactions between the host water molecules and the entrapped guest molecules. There is no bonding between the guest and host molecules. Methane, ethane, carbon dioxide form the sI hydrate and argon, oxygen form sII hydrates. $\mathrm{CO}_{2}$ molecules form structure I hydrate and occupy most of the tetrakaidecahedral cages and a fraction of smaller dodecahedral. Gas hydrates are nonstoichiometric compounds since all available cages within the lattice structure are not completely occupied for stability.

Table 1.1 Hydrate crystal structure ${ }^{17}$

\begin{tabular}{|c|c|c|c|c|c|c|c|}
\hline \multirow{2}{*}{$\begin{array}{c}\text { Property } \\
\text { Cavity }\end{array}$} & \multicolumn{2}{|c|}{ Structure I } & \multicolumn{2}{|c|}{ Structure II } & \multicolumn{3}{|c|}{ Structure H } \\
\hline & Small & Large & Small & Large & Small & Medium & Large \\
\hline Description & $5^{12}$ & $5^{12} 6^{2}$ & $5^{12}$ & $5^{12} 6^{4}$ & $5^{12}$ & $4^{3} 5^{6} 6^{3}$ & $5^{12} 6^{8}$ \\
\hline Cavities/unit cell & 2 & 6 & 16 & 8 & 3 & 2 & 1 \\
\hline $\begin{array}{c}\text { Water } \\
\text { molecules/unit cell }\end{array}$ & \multicolumn{2}{|c|}{46} & \multicolumn{2}{|c|}{136} & \multicolumn{3}{|c|}{34} \\
\hline $\begin{array}{c}\text { Average cavity } \\
\text { radius }(\AA)\end{array}$ & 3.95 & 4.33 & 3.91 & 4.73 & 3.94 & 4.04 & 5.79 \\
\hline
\end{tabular}




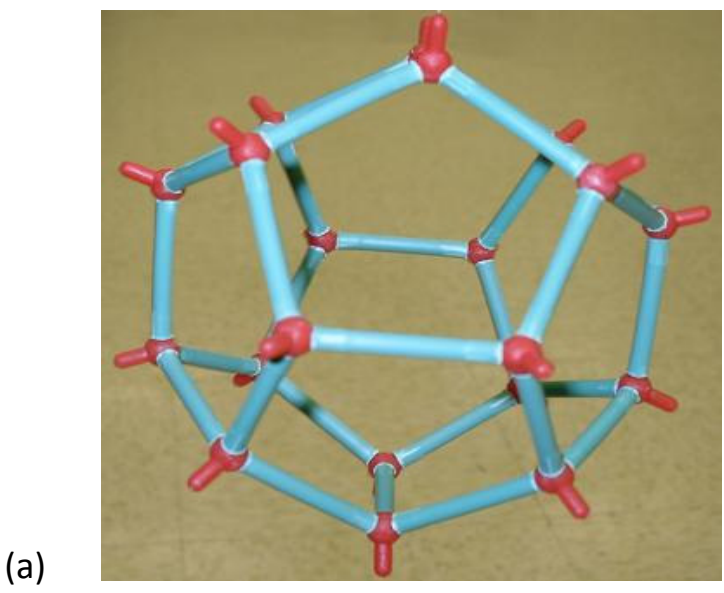

(b)

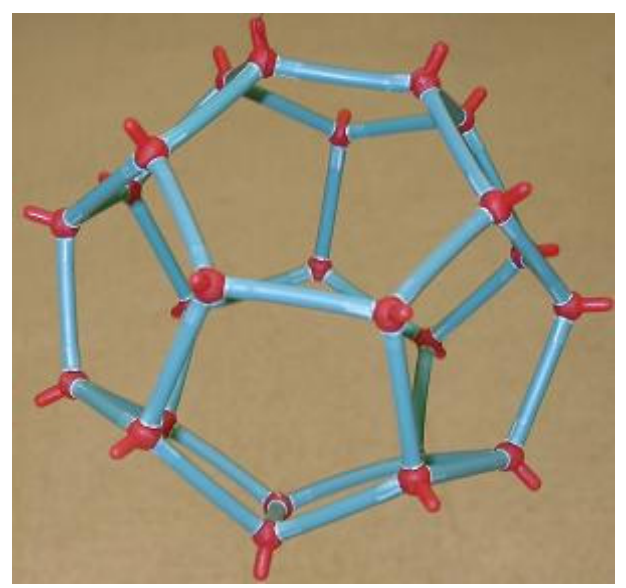

Figure 1.3 Cavities of Structure 1: (a) pentagonal dodechaderon (small cage, $5^{12}$ ) (b) tetrakaidecahedran (large cage, $5^{12} 6^{2}$ )

(a)

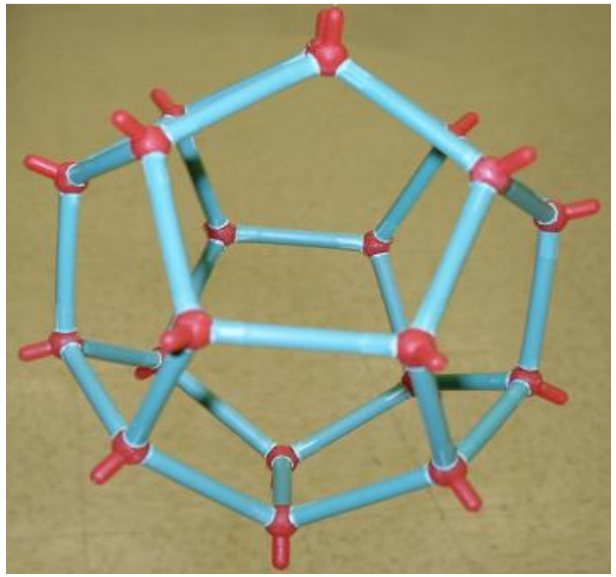

(b)

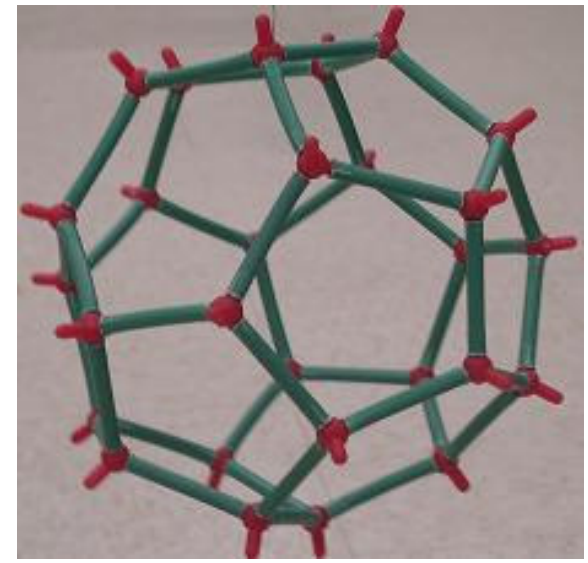

Figure1.4 Cavities of Structure II: (a) pentagonal dodechaderon (small cage, $5^{12}$ ) (b) hexakaidecahedron (large cage, $5^{12} 6^{4}$ ) 
(a)

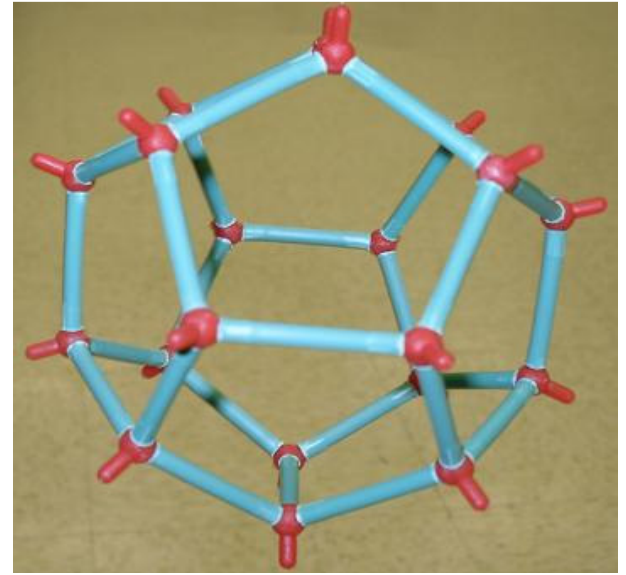

(b)

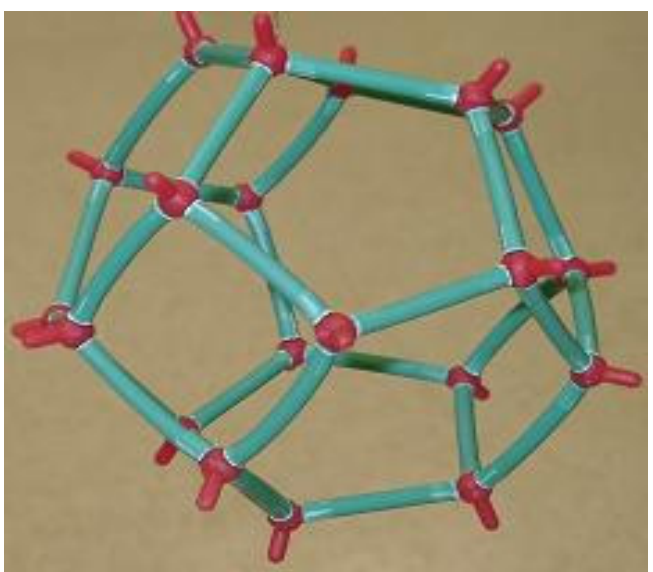

(c)

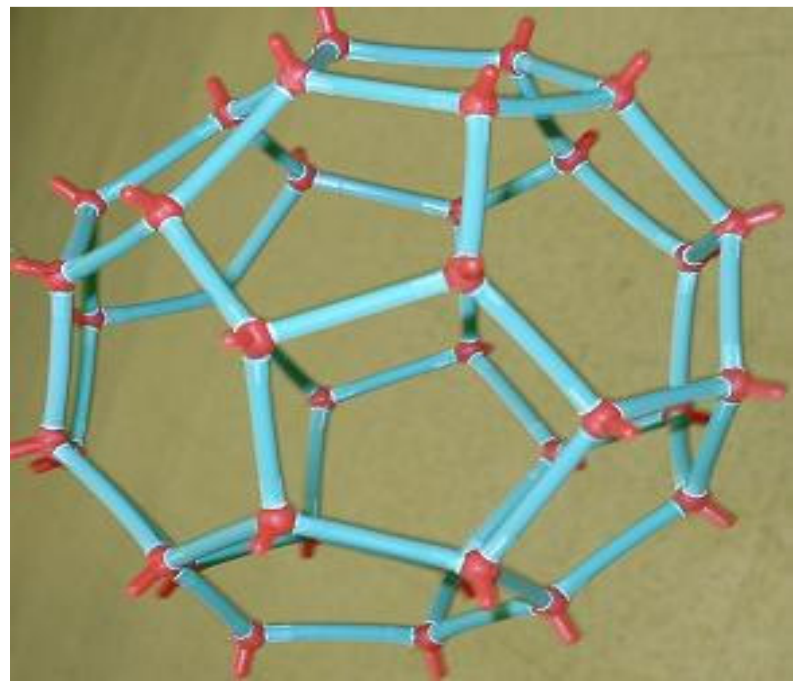

Figure 1.5 Cavities of Structure H: (a) pentagonal dodechaderon (small cage, $5^{12}$ ) (b) irregular dodechaderon (medium cage, $4^{3} 5^{6} 6^{3}$ ) (c) icosahedron (large cage, $5^{12} 6^{8}$ ) 


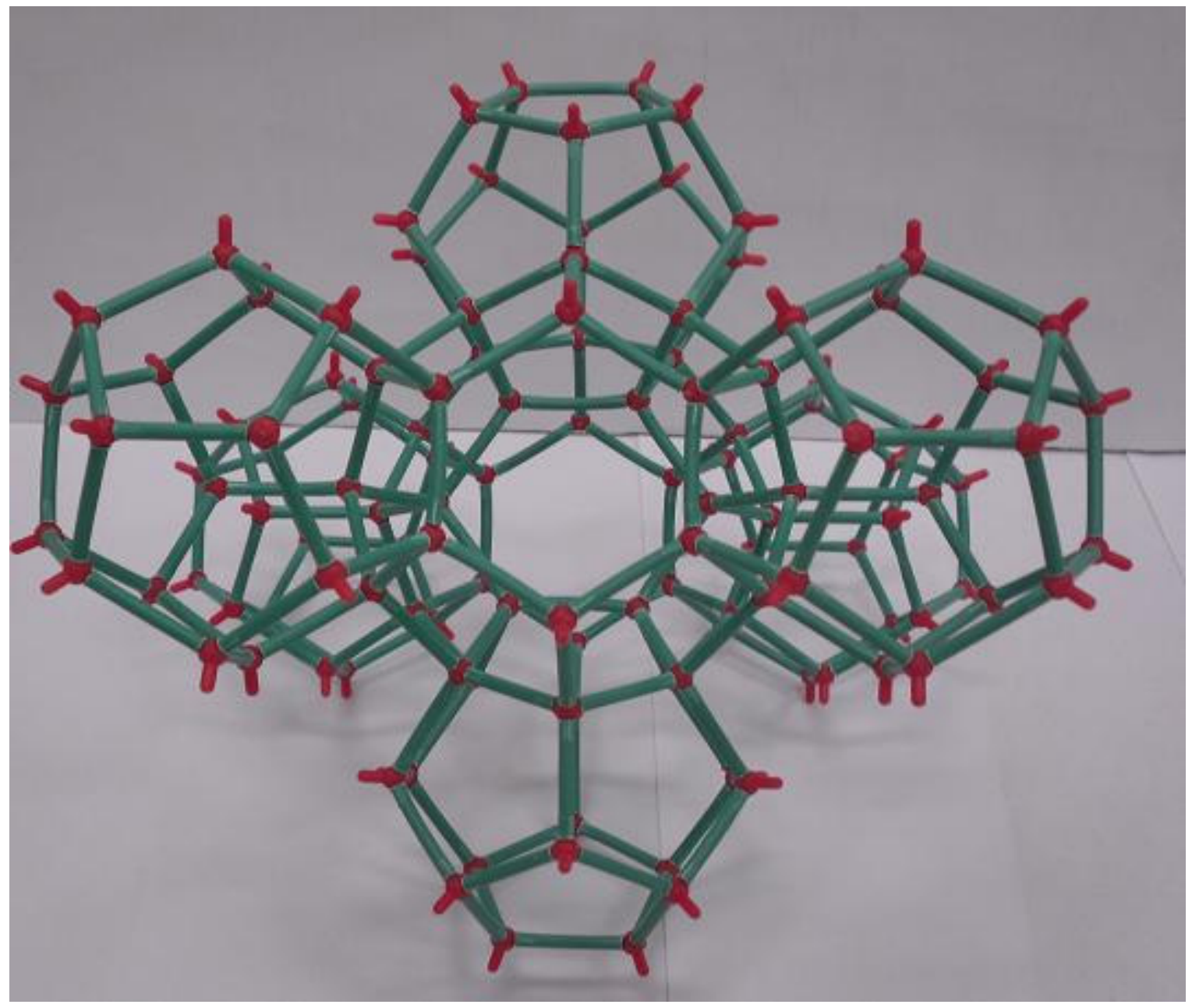

Figure 1.6 Lattice structure of Structure I hydrate. 


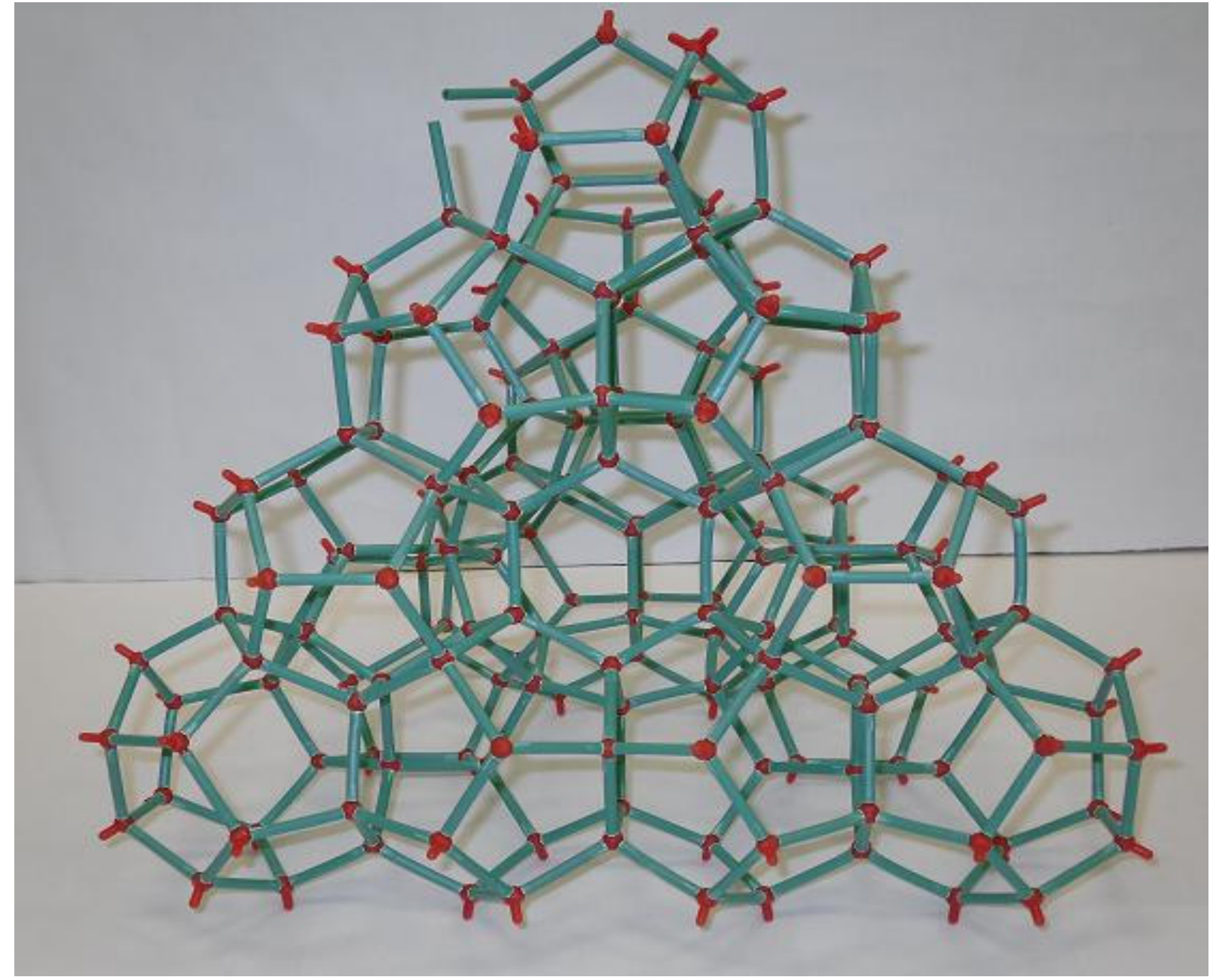

Figure1.7 Lattice structure of Structure II hydrate 


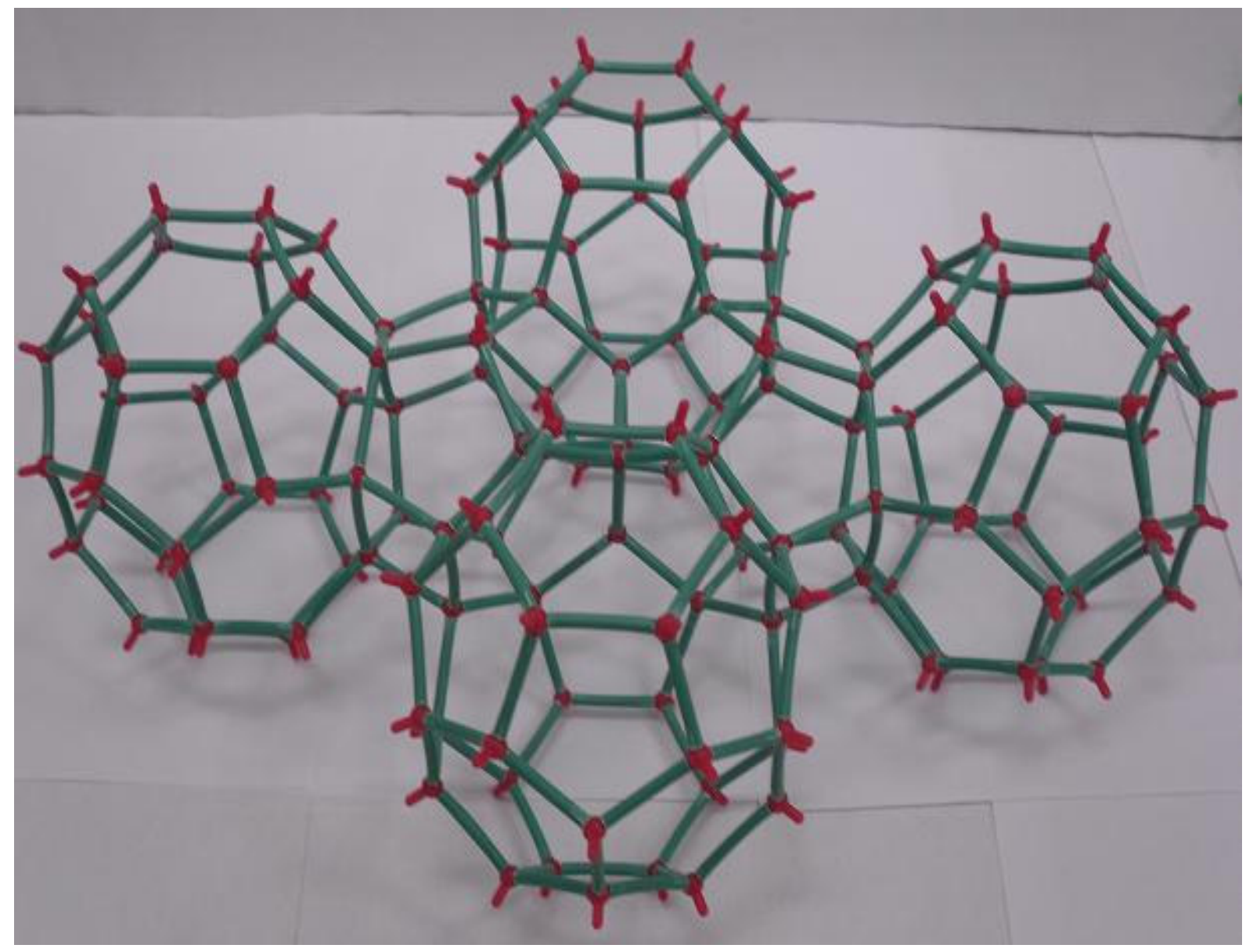

Figure 1.8 Lattice structure of Structure H hydrate 


\subsubsection{Lattice structure used in this study}

During the sixty's extensive series of crystallographic studies were performed on sI and sII hydrates by Jeffrey and coworkers ${ }^{20,22}$. Diverse physical techniques were used to study the hydrate structure. At first, XRD (single crystal and powder) was used, followed by dielectric techniques and NMR spectroscopy. Applying Raman spectroscopy and single crystal X-ray diffraction for composition and guest distribution of clathrate hydrate emerged in the last decade. In this work, the host lattice fractional positional parameters reported by McMullan and Jeffery $^{22}$ were selected to represent the oxygen positions within structure I and for structure II by Mark and McMullan ${ }^{20}$. The experimental structure of an isolated water molecule $(r(\mathrm{OH})=$ $\left.0.9752 \AA, \mathrm{HOH}=\angle 104.52^{\circ}\right)$ or the simple point charge $(\mathrm{SPC})$ model of water $(r(\mathrm{OH})=1.0 \AA$, $\mathrm{HOH}=\angle 109.47^{\circ}$ ) can be used as a desired geometry of water as proposed by Berendson et al. ${ }^{23}$

\subsubsection{Proton Placement}

The water proton distribution that forms the clathrates must be known to understand the configurational characteristics of guest-host interactions inside the cavities. Unfortunately, it is very difficult to measure the proton positions from the conventional diffraction studies. An algorithm was developed by the Sparks $^{24}$ to randomly assign the proton to their respective positions with conforming to Bernal-Fowler Rules ${ }^{25}$ and the constraint that the net dipole of the whole clathrates hydrate structure system should be zero. Nearly, half a million configurations were generated for each clathrate structure and desired water molecule geometry and the resulting configuration with the lowest net dipole moment was then selected as a valid proton 
assignment. The Bernal-Fowler Rules further refined by Rahman and Stillinger ${ }^{26}$ are outlined below:

1) Water clathrate host lattice consists of intact (non-dissociated) water molecules.

2) The oxygens form the host lattice with very nearly tetrahedral coordination.

3) Each hydrogen bond between two neighboring oxygens is made up of a single proton covalently bonded to one of the oxygens and hydrogen bonded to the other.

4) All proton configurations satisfying above three conditions are equally probable.

\subsection{Overview of Previous Theoretical work}

Gas hydrates thermodynamics are important in exploring the gas hydrates reservoirs, $\mathrm{CO}_{2}$ sequestration on ocean bed and also swapping process of $\mathrm{CH}_{4}$ hydrate with $\mathrm{CO}_{2}$. With the experimental limitations, studies on the development of thermodynamic model for the prediction of phase behavior of the gas hydrates are of great importance. An initial statistical thermodynamics model to determine the gas hydrates properties was suggested by Barrer and

Straut $^{27}$. Van der Waals and Platteeuw ${ }^{28}$ in a similar yet more successful approach proposed a basic model corresponding to the three dimensional generalization of ideal localized adsorption, derived the grand canonical partition function for water, with the following assumptions.

1) Each cavity can contain at most one gas molecule.

2) The interaction between a gas and water molecule can be described by a pair potential functions, and the cavity can be treated as perfectly spherical. 
3) The free energy contribution of the water molecules is independent of the mode of dissolved gases (cage distortions are neglected)

4) There is no interactions between the gas molecules in different cavities and the guest molecule interact with the nearest neighbor water molecules (guest-guest interactions are neglected).

The van der Waals and Platteeuw model has been widely used in various applications in gas hydrate systems. It uses statistical thermodynamics to predict the macroscopic property like chemical potential of the hydrate, using microscopic properties like intermolecular potentials. The important term in the van der Waals and Platteeuw model is the Langmuir constant. The Langmuir constant accounts for the configurational intermolecular interactions between the guest gas molecule and all the surrounding host water molecules in the clathrates hydrate lattice. The expression for Langmuir constant for asymmetrical guest molecule is given by Equation 1.1. Langmuir constant can be computed if a total potential function, $\Phi(r, \theta, \phi, \alpha, \beta, \gamma)$, for these guest-host interactions in a cavity is known, which is the key term to predict the phase equilibrium and cage occupancy of gas hydrates accurately.

$$
C_{j i}=\frac{1}{k T} \int_{0}^{2 \pi} \int_{0}^{\pi} \int_{0}^{R} \exp \left(-\frac{\Phi(r, \theta, \phi, \alpha, \beta, \gamma)}{k T}\right) r^{2} \sin \theta d r d \theta d \phi d \alpha d \beta d \gamma
$$

In their original work, van der Waals and Platteeuw ${ }^{28}$ applied the Lennard-Jones and Devonshire cell theory, which is referred as the LJD approximation in this work. They assumed that the guest-host interactions can be represented by a guest molecule at a distance $r$ from the cavity center in a spherically symmetrical potential $\Phi(r)$ induced by the host molecules. The 
model assumes that $\mathrm{W}(r)$ is a suitable average of $\Phi(r, \theta, \phi)$ without actually averaging it. The smoothed cell Langmuir constant becomes:

$$
C_{j i}^{*}=\frac{4 \pi}{k T} \int_{0}^{R} \exp \left(-\frac{W(r)}{k T}\right) r^{2} d r
$$

The binary interaction between a guest molecule and a water molecule of the cavity was represented by the Lennard-Jones 6-12 spherically symmetric potential. The van der Waals and Platteeuw model works well for monatomic gases and quasispherical molecules but it couldn't predict the dissociation pressure for non-spherical and polyatomic molecules quantitatively. McKoy and Sinanoglu ${ }^{29}$ demonstrated that better results could be obtained by using the Kihara potential function with a spherical core. The Kihara potential parameters were determined by second virial coefficient data. Marshall et al. ${ }^{30}$ and Nagata and Kobashi ${ }^{31}$ estimated the potential parameters by fitting the experimental data for methane, argon and nitrogen hydrates. These estimated parameters were used to predict the hydrate formation pressures of ternary mixtures. Parrish and Prausnitz ${ }^{32}$ later extended the van der Waals and Platteeuw model with fitted Kihara parameters to predict the dissociation pressures of gas hydrates formed by multi-component guest mixtures. This method has gained wide acceptance and been used in modified forms. ${ }^{17,33,34}$ However, as more experiments were performed for different gas mixtures and temperatures, the van der Waals and Platteeuw model with the parameters set of Parrish and Prausnitz ${ }^{32}$ in some cases failed to accurately predict equilibrium pressures $^{58}$. The ability of these fits to predict the phase equilibrium beyond the range of the fit is limited. 
The main reasons for the errors in LJD approximation to predict the phase equilibrium accurately are cavity asymmetry and contributions from multi shell water hosts. John and Holder modified the van der Waals and platteeuw model:

1) The choice of the cell size used in the LJD theory. ${ }^{35}$

2) The addition of terms to account for the contribution of second and subsequent water shells to the potential energy of the guest-host interactions in clathrates hydrates. $^{36}$

John and Holder ${ }^{36}$ studied the choice of the cell size used in the LJD theory and provided the optimal cell sizes and coordination numbers for different cavities to equalize the smoothed cell potential and discretely summed potential. However, these parameters are not consistent with the crystallographic structure of clathrates hydrate. John and Holder ${ }^{36}$ proposed further modifications and included the interactions between a guest molecule and the second and third neighbor water molecules contributions in the potential energy calculations. The Langmuir constant is redefined as

$$
C_{j i}^{*}=\frac{4 \pi}{k T} \int_{0}^{R} \exp \left(-\frac{W_{1}(r)+W_{2}(r)+W_{3}(r)}{k T}\right) r^{2} d r
$$

The magnitudes of the second interactions are significant and can change the Langmuir constant to several orders of magnitude influencing the phase equilibrium predictions. They carried out more precise calculations for Langmuir constant using the crystallographic locations of the host water molecules and modeling binary guest-host interactions by Kihara-type potentials. They compared the Langmuir constant results to those obtained by LJD approach. The variation of Langmuir constant obtained from two methods is dependent on the Kihara 
effective size and energy parameters. John and Holder proposed to use an empirical aspherical correction to Langmuir constant due to the restricted motion of the gas molecule and it is given as

$$
C_{j i}=C_{j i}{ }^{*} Q^{*}
$$

where $C_{j i}{ }^{*}$ is the spherical cell Langmuir constant given in Equation 1.3 and $Q^{*}$ is an empirical function that corrects the Langmuir constant due to the restricted motion of the spherical gas molecule. This correction, $Q^{*}$, accounts for all nonidealities in the molecular interactions between the enclathrated gas and the hydrate lattice water molecules in their generalized model for predicting equilibrium conditions for gas hydrates. John and Holder ${ }^{61}$ based on some trends with molecular properties hypothesized the following empirical correlation for $Q^{*}$ as

$$
Q^{*}=\exp \left[-a_{o}\left[\omega\left(\frac{\sigma}{R-a}\right)\left(\frac{\epsilon}{k T_{o}}\right)\right]^{n}\right]
$$

where $a_{o}$ and $n$ are empirical parameters which depends on particular cavity and $a, \epsilon$ and $\sigma$ are Kihara potential parameters(see Equation 2.25). The values of $a_{o}$ and $n$ are fitted to experimental dissociation pressure.

The Kihara parameters used above were obtained by fitting to the viscosity and second virial coefficient data and predicted the phase equilibria of gas hydrates ${ }^{61}$, but they have effectively introduced new empirically fitted parameters such as the cell radius into the model. The improvements, however, were not found to be striking because the Kihara potential is not giving a fundamentally accurate description of the potential field in the cavities ${ }^{37}$ and according to Avlonitis et al. ${ }^{38,39}$, the effect of non idealities had been overestimated. Tester et al. ${ }^{40}$ 
calculated the Langmuir constant by Monte Carlo simulations which avoided the use of the LJD approximation, the potential energy was calculated from Metropolis et al. ${ }^{41}$ technique. This method gives erroneous computed Langmuir constants owing to possible failure of assumptions made to obtain the Langmuir constant ${ }^{42}$.

Many of the previous models were semi empirical fitting methods; they are the combinations of the van der Waals and Platteeuw statistical model and experimental phase equilibria data fitting. This models work well in the experimental regime in the fitted data range and fails when extended outside the regime. The spherical symmetric LJD assumption simplifies the configurational integral to a one-dimensional integral, because of this the crystallographic structure has not sufficiently taken in to account resulting in the prediction of macroscopic properties.

In the original van der Waals and Platteeuw ${ }^{28}$ model, the reference chemical potential difference, $\Delta \mu_{w}{ }^{\beta-L, \alpha}\left(T_{o}, 0\right)$, which is the difference between the theoretical empty hydrate and liquid water at its reference state $\left(T_{o}=273.15 \mathrm{~K}\right.$ and $\left.0 \mathrm{kPa}\right)$ was assumed to be known and is not affected by any enclathrated guest molecule. They assumed a non-distortion of hydrate lattice in the model. This assumption requires that the volume of the empty hydrate lattice must be equal to the volume of the hydrate at equilibrium. However, recent studies have proved that there is a lattice distortion when the guest size or temperature changes ${ }^{61,70}$. Holder et al. ${ }^{61}$ first questioned the assumption of $\Delta \mu_{w}{ }^{\beta-L, \alpha}\left(T_{o}, 0\right)$ as a constant and proposed the idea of the lattice distortion. They suggested that the reference chemical potential difference vary with guest molecules. Hwang et al. ${ }^{71}$ performed the molecular dynamics simulations on the unit cell of gas hydrate with different guests. They performed the calculations on the spherical guests in order to avoid the asymmetry of the guest and their results showed that the lattice size giving the 
minimum total energy varied from guest to guest. The lattice constant increases as the guest size is increased. Lee and Holder $^{73}$ developed a new algorithm to predict hydrate equilibrium with variable reference chemical potential. In their algorithm, an empirical correlation developed by Zele et al. ${ }^{72}$ was applied to get the cavity radius as a function of the reference chemical potential, $\Delta \mu_{w}{ }^{\beta-L, \alpha}\left(T_{o}, 0\right)$, and is given as:

$$
R=A+B \times \Delta \mu_{w}^{\beta-L, \alpha}\left(T_{o}, 0\right)
$$

where $R$ is the radius and is in $\AA, A$ and $B$ are constant for three water shells of each type of cavity. They calculated the reference chemical potential for different guests using the above algorithm and their results shows that the reference chemical potential increases as the size of the guest increases.

Bazant and Trout ${ }^{43}$ proposed a mathematical method to determine the spherically averaged intermolecular potentials from the temperature dependent Langmuir constant. The spherical-cell formula for the Langmuir constant verses temperature can be viewed as a nonlinear integral equation for the cell potential, and exact potential forms can be found as a solution to this integral equation. Anderson et al.$^{60}$ used the Bazant and Trout ${ }^{43}$ mathematical model to predict phase equilibria of multicomponent gas hydrate systems. They found the potential well depths and negative energy volumes for 16 single component hydrate system using the central well solution. They calculated the mixture phase diagrams for ethane, methane and cyclopropane and also predicted the structural transition for methane-cyclopropane hydrate system.

Sparks and Tester $^{44}$ presented a rigorous numerical model for calculating guest-host and guest-guest intermolecular potential energy contributions for an infinite water clathrate lattice 
and was used to characterize the quantitative extent of these effects on the configurational partition function and the three-dimensional Langmuir constant. They found that guest-guest interactions and the subsequent water shell interactions do indeed have significant effect on the Langmuir constant values. The spherical LJD approximation was avoided by Sparks ${ }^{24}$ in his dissertation and performed multi-dimensional integral, accounting the asymmetries of the host lattice using the crystallographic structural data. Cao et al. ${ }^{45,46}$ evaluated Langmuir constant numerically as a six-dimensional integral for methane hydrate. Most of the previous models compute Langmuir constant from the Kihara potential model and the parameters of the Kihara potential are empirically regressed from experimental phase equilibrium data. These potentials have very little physical meaning and were not able to predict the phase equilibrium well for the multi component gases. To predict more accurate phase equilibria and for the molecular simulation studies of the hydrates there is a need of physically-based intermolecular potentials. Cao et al. ${ }^{47}$, Klauda and Sandler ${ }^{48}$ and Anderson et al. ${ }^{49}$ computed guest-host inter molecular potentials from $a b$ initio quantum mechanical calculations. With these potentials they computed Langmuir constant and further calculated phase equilibrium and cage occupancies for methane hydrate. Ab initio quantum mechanical calculations seem to provide an independent means to directly obtain accurate intermolecular potentials.

The ab initio calculations for $\mathrm{CO}_{2}-\mathrm{H}_{2} \mathrm{O}$ complex was first studied by Goldmann ${ }^{50}$ using self-consistant-field methods (Hartree-Fock method) which predicted a "T-shaped", planar complex, between the carbon of $\mathrm{CO}_{2}$ and oxygen of $\mathrm{H}_{2} \mathrm{O}$ forming a van der Waals bond. This T-shaped geometry was confirmed by Peterson and Klemperer ${ }^{51}$ using molecular-beam electronic resonance methods. Mehler ${ }^{52}$ performed the $a b$ initio calculations on the $\mathrm{CO}_{2}-\mathrm{H}_{2} \mathrm{O}$ dimer with 6-31G basis set. They have used the nonorthogonal group function (NOGF) 
approximation for the analysis of noncovalent interactions instead of using the standard selfconsistent-field molecular orbital (SCF-MO) wave function. Block et al. ${ }^{53}$ performed ab initio calculations at second order Møller-Plesset perturbation theory (MP2) with basis set of 6-31+G (2d, 2p). Makarewicz et al. ${ }^{54}$ (1993) calculated the potential energy surface of $\mathrm{H}_{2} \mathrm{O}-\mathrm{CO}_{2}$ complex using $a b$ initio calculations with MP2/6-31++G(2d,2p) basis set. Kieninger and Ventura $^{55}$ performed MP2/6-31++G (2d, 2p), MP4, QCISD (T) and density functional calculations on the charge-transfer complex between carbon dioxide and water. The estimated binding energy was $-2.87 \pm 0.2 \mathrm{kcal} / \mathrm{mol}$ corresponding to the optimized minimum energy structure. All these previous ab initio calculations were performed to locate the minimum energy structure and to estimate the vibrational bond frequencies. All these studies predicted a T-shaped planar structure as shown in Figure 1.8, with the carbon atom attached to oxygen of water, to be a global equilibrium configuration. But all of these calculations neglected the basis set superposition error (BSSE).

The intermolecular energy functions used by Sun and Duan ${ }^{56}$ were based on ab initio PES calculations carried out by Sadlej et al. ${ }^{57}$. Sadlej et al. applied supermolecular MollerPlesset perturbation theory (MPPT) to calculate the potential energy surface of the carbon dioxide-water complex with various quality basis set with the largest being $S(s p d f)$.They have used the counterpoise method to reduce the deviation caused by BSSE. They found two minima, global minima for the $\mathrm{T}$-shaped structure and local minima for the H-bonded arrangement $\mathrm{OCO} \cdots \mathrm{HOH}$. Danten et al. ${ }^{59}$ optimized the complex at the MP2 level with higher basis set of aug-cc-pVTZ and aug-cc-pVDZ and calculated the BSSE corrected binding energies as -2.6 and $-2.3 \mathrm{kcal} / \mathrm{mol}$ respectively. 

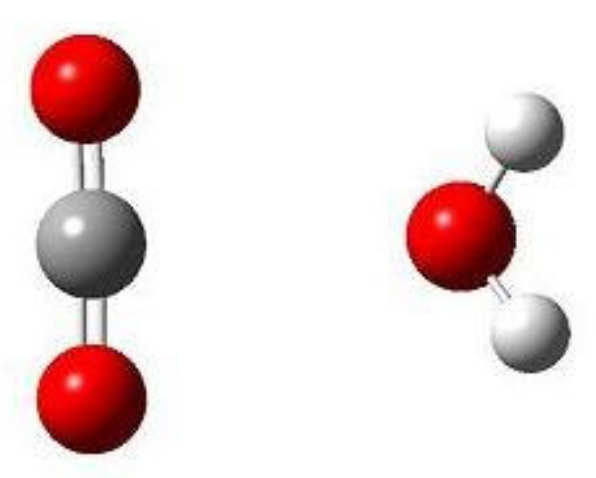

Figure1.9 T-shaped structure of $\mathrm{CO}_{2}-\mathrm{H}_{2} \mathrm{O}$ complex

Cao et al. ${ }^{47}$ computed the methane-water potential energy hypersurface via ab initio methods. They computed the $\mathrm{CH}_{4}-\mathrm{H}_{2} \mathrm{O}$ binding energy at 18,000 points describing the position and orientation between $\mathrm{CH}_{4}$ and $\mathrm{H}_{2} \mathrm{O}$ molecules. They developed a method in which all these 18,000 points were computed at MP2/ 6-31G++G $(2 \mathrm{~d}, 2 \mathrm{p})$ basis set and corrected to the ccpVQZ basis set level with 100 points calculation to reach accuracies of less than $0.1 \mathrm{kcal} / \mathrm{mol}$. Cao et al. $^{45}$ demonstrated the ability of this ab initio potential to accurately predict methane hydrate dissociation pressure across a large range of temperatures, but it gives unreasonable cage occupancy. Before the calculation of Langmuir constant they performed spherical average on the intermolecular potentials using Boltzmann averaging algorithm which causes the loss of ab initio potential quality.

Klauda and Sandler ${ }^{48}$ showed that many-body interactions should be accounted for when applying computed potentials to the hydrate clathrates system. They performed ab initio calculations directly on the quarter cell (divided the hydrate in to four sections) with $6-31++\mathrm{G}$ (3d, 3p) basis set. The interaction energies between the guest and each section of the lattice is calculated and then summed to estimate the interaction energies of the guest and the full cage. They also calculated the interaction energies of methane with each water molecules separately 
for 20 water molecules and then summed, these summed energy is far from the interaction energies results for the full, half and quarter cages indicating the importance of many-body effects in the hydrates. They have not included the interaction between the guest and the outer water shells in the Langmuir constant calculations.

Recently, Anderson et al. ${ }^{49}$ performed high level $a b$ initio quantum mechanical calculation to determine the intermolecular potential energy surface between argon-water to predict the phase equilibria for the argon hydrate and mixed argon-methane hydrate system. They used the site-site potential model to fit the ab initio potentials for $\mathrm{CH}_{4}-\mathrm{H}_{2} \mathrm{O}$ improving the work of Cao et al. $^{45}$ in predicting the cage occupancies. The intermolecular potentials were corrected for many body interactions and also included the interaction between the guest and the outer water shells still the fourth shell. Similar to Anderson et al. ${ }^{49}$, Sun and Duan ${ }^{56}$ predicted the $\mathrm{CH}_{4}$ and $\mathrm{CO}_{2}$ phase equilibrium and cage occupancy from ab initio intermolecular potentials. The ab initio calculations were taken from Sadlej et al. ${ }^{57}$ for the $\mathrm{CO}_{2}$ $\mathrm{H}_{2} \mathrm{O}$ complex. They used atomic site-site potential model to fit the ab initio potentials.

Proper determination of the form of the intermolecular interaction potential is also necessary both to compute equilibrium thermodynamic properties and to perform dynamics molecular simulations of kinetic phenomena such as diffusion and hydrate crystal nucleation and its growth and decomposition. 


\subsection{Motivation and Scope of Work}

\subsubsection{Hydration number}

Hydration number is the average number of water molecules per guest molecule in the hydrate. Hydration number and cage occupancies are important as it tells the amount of gas stored in the hydrate. Composition of the hydrate at in-situ temperature and pressure must be known in order to fully understand the thermodynamics and the kinetics of the gas hydrate formation and decomposition. A variety of approaches has been used to measure the hydrate cage occupancies and the hydration number. Cage occupancies have been reported using spectroscopic measurements. Classical approach includes the application of the ClausiusClapeyron equation to the water-hydrate-gas equilibrium data. For fully occupied large, $\theta_{L}=1$ and small cages, $\theta_{s}=1$, of structure I gas hydrate the hydration is of 5.75 . Bozzo et al. ${ }^{62}$ calculated the hydration number from the dissociation enthalpies of $\mathrm{CO}_{2}$ hydrate using the Clausius- Clapeyron equation and gave the value of 7.23.

Nuclear magnetic resonance (NMR) and Raman spectroscopy has been used to measure the relative cage occupancies, in which the integrated signal intensity ratios of the guests in the two cavities are measured. Hydration numbers can be calculated from the relative cage occupancies obtained by spectroscopic measurements and the free energy difference between ice and the hypothetical empty hydrate lattice $(\Delta \mu) .{ }^{63,64}$ Sum et al. ${ }^{64}$ used Raman spectroscopy to measure the cage occupancies of the methane-carbon dioxide mixture gas hydrate. They also measured the Raman spectra for $\mathrm{CO}_{2}$ single hydrate, and Raman spectroscopy measurements were not able to distinguish the large and small cage occupancy for $\mathrm{CO}_{2}$ hydrate. They reported that the guest $\mathrm{CO}_{2}$ appeared to occupy only the large cavities as they have not seen any splitting 
of the Raman bands representing the different environments for guest to occupy small cavities and large cavities. But the neutron diffraction studies by Ikeda et al ${ }^{65}$ and the X-ray diffraction studies by Udachin et al. ${ }^{66}$ of pure $\mathrm{CO}_{2}$ hydrates found that the carbon dioxide also occupies the small cavity $\left(5^{12}\right)$.

The cage occupancies determined by the Henning et al. ${ }^{67}$ from neutron diffraction studies for the $\mathrm{CO}_{2}$ guest were more than $95 \%$ for the large cavities, and for the small cages is in the range of $60 \%$ to $80 \%$. This gives the hydration numbers between 6.05 and 6.67 . They prepared the sample at temperatures between $263 \mathrm{~K}$ and $278 \mathrm{~K}$ with pressures well above the equilibrium pressures, around $60 \mathrm{~atm}$. The cage occupancies reported by Udachin et al. ${ }^{66}$ from the single crystal X-ray diffraction studies were $100 \%$ for the large cage $\left(\theta_{L}\right)$ and $71 \%$ for the small cage $\left(\theta_{S}\right)$; this yields the hydration number of 6.20 . They prepared the crystal at temperature $276 \mathrm{~K}$ in the presence of excess liquid $\mathrm{CO}_{2}$ and pressure almost twice that of the equilibrium condition, at $38 \mathrm{~atm}$. All the above $\mathrm{CO}_{2}$ hydrate samples prepared for determining the cage occupancies and hydration numbers by experimental measurements were well above the equilibrium pressures and these higher pressures during the synthesis produce higher occupancies. Ripmeester and Ractliff ${ }^{68}$ prepared a sample under equilibrium conditions, at temperature $268 \mathrm{~K}$ and pressure of 9.9 bar, gave a lower limit to the hydration number of 7.0 for $\mathrm{CO}_{2}$ hydrate. They used solid state NMR to measure the relative cage occupancy, $\theta_{S} / \theta_{L}$, of 0.32 and assumed a $\Delta \mu_{w}{ }^{0}$ value of $1297 \mathrm{~J} / \mathrm{m}$ for a hydration number calculation.

Sun and Duan ${ }^{56}$ predicted the hydration numbers from the $a b$ initio intermolecular potentials for $\mathrm{CO}_{2}$ hydrate at different temperatures and pressures. They predicted a hydration number in between 6.412 and 6.548 at a temperature between 268 and $273.65 \mathrm{~K}$ and equilibrium pressures, where as the lower limit given by Ripmester and Ractliff ${ }^{68}$ is of 7.0. 
This means that Sun and Duan ${ }^{56}$ model over estimated the cage occupancies of the $\mathrm{CO}_{2}$ hydrate. Klauda and Sandler ${ }^{48}$ predicted the composition of the guest in the methane-carbon dioxide mixed hydrate. They used the van der Waals and Platteeuw ${ }^{28}$ model along with an $a b$ initio LJ potential in estimating the composition of the guest in the hydrate. Their predictions over estimates the overall composition of methane hydrate in the hydrate phase at mixed temperature compared to the experimentally measured guest composition by Ohagaki et al. ${ }^{69}$ Even the empirically fit Sloan/Kihara potential over-estimates the occupancies for the pure carbon dioxide hydrate and methane-carbon dioxide mixed hydrate. ${ }^{28}$ There are not much of experimental measurements or the prediction methods that describe the cage occupancies of $\mathrm{CO}_{2}$ hydrate accurately at equilibrium conditions.

Recent work by Park et al. ${ }^{13}$ on the replacement of methane with $\mathrm{CO}_{2}$ in naturally occurring gas hydrates has shown some potential, but the connection between the molecular level events that occur during this replacement is not yet known. Most of the hydrate simulations have assumed that the hydrate deposit is a pure methane hydrate, but in nature there is a great possibility of encountering complex gas hydrate mixtures. The current state of mixed hydrate thermodynamics is not well suited for accurate thermodynamic predictions of the methane-carbon dioxide mixed hydrate. The most common potential used for the carbon dioxide thermodynamic modeling is the spherical Kihara potential; these potential parameters were obtained by fitting to the experimental data. The use of this potential to predict the mixed hydrate thermodynamics results in inaccurate predictions. Sloan has regressed the Kihara potential for $\mathrm{CO}_{2}$ hydrate by empirically fitting to the experimental data ${ }^{17}$. Ikeda et al. ${ }^{65}$ reported that the asymmetry of the $\mathrm{CO}_{2}$ molecule leads to the thermal vibrations of the host water atoms of the $\mathrm{CO}_{2}$ hydrate. Therefore, the asymmetric nature of the $\mathrm{CO}_{2}$ guest molecule 
must be taken in account for accurate modeling of the $\mathrm{CO}_{2}$ hydrate and also for the carbon dioxide and methane mixed hydrate. A theoretically-based model is needed which can predict the mixed hydrate thermodynamics with a stronger connection to the physics of the guest host interaction.

The two most important properties involved in the hydrate equilibria calculations are the Langmuir constant, $C$, and the reference chemical potential difference, $\Delta \mu_{w}{ }^{o}$. Previous semi empirical models calculated the Langmuir constant for the $\mathrm{CO}_{2}$ hydrate by fitting the experimental data by assigning a specific value for reference chemical potential difference. When determining the reference chemical potential difference by applying the LJD approximation, Langmuir constant is calculated by assuming that a hydrate cavity could be described as a uniform distribution of water molecules smeared over a sphere of radius. A better model is needed which can simultaneously incorporate these two parameters to give more accurate model, one that can interpolate/extrapolate the experimental data and also represent the physical reality. The Langmuir constant will be determined by considering the asymmetry of the guest molecule and the guest-host intermolecular potentials that are determined independently by ab initio potential energy surface.

\subsubsection{Objectives of this study}

The goal of this work is to determine the effective interaction energies between the $\mathrm{CO}_{2}$ guest molecule and the water host molecules by developing guest-host pair potential using an $a b$ initio potential energy surface. These $a b$ initio intermolecular potentials will be used to calculate the Langmuir constant, including the contributions of interactions between the $\mathrm{CO}_{2}$ 
guest and the host molecules from first water shell to fourth water shell. Using these Langmuir constants, the phase equilibrium and cage occupancy of the $\mathrm{CO}_{2}$ hydrate can be predicted and extended to the $\mathrm{CO}_{2}-\mathrm{CH}_{4}$ mixed hydrate predictions using the cell potential method ${ }^{60}$.

Furthermore, the ab initio potentials can be used in molecular dynamics simulations to study the stability and also the lattice distortion caused by non-ideality of the $\mathrm{CO}_{2}$ molecule. 


\subsection{References}

1. Powel, H.J.M. J. Chem. Soc. 1948, 61.

2. Davy, H. Phi. Trans. Soc. London 1811, 101, 1.

3. Pristley, J. Experiments and observations on different kind s of air, and other branches of natural philosophy, connected with the subject; Thomas Perrson: Birmingham, 1790; Vol 2.

4. Wroblewski, S. (1882b) On the composition of the hydrate of the carbonic acid. Acad. Sci. Paris, ibid., pp. 954-958 (Original language French)

5. Wroblewski, S. (1882c) On the laws of solubility of the carbonic acid in water at high pressures. Acad. Sci. Paris, ibid., pp. 1355-1357 (Original language French)

6. Hammerschmidt, E.G. Ind. Eng. Chem. 1934, 26, 851.

7. Kvenvolden, K. A. Chem. Geol. 1988, 71, 41.

8. Makogon, Y.F. La Recherche 1987, 18, 1192.

9. Trofimuk, A.A.; Makogon, Y.F.; Tolkachev, M.V. Geologiya nefti I Gaza 1981, 10, 15.

10. Soloview, V. A. Russian Geol.Geophys. 2002, 43, 648

11. Klauda, J.B.;Sandler, S. I. Energy \& Fuels 2005, 19, 459.

12. Holder, G. D.; John, V. T.; Yen, S. "Geological implications of gas production from In-situ gas hydrates"; SPE/DOE symposium on unconventional gas recovery, 1980.

13. Park, Y.; Kim, D. Y.; Lee, J. W.; Huh, D. G.; Park, K. P.; Lee, J.; Lee, H. Preecedingd of the National Academy of Sciences of the United States of America 2006, 103, 12690.

14. Bardhun, A. J.; Towlson, H.E.; Ho, Y. C. AIChE J 1962, 8, 176.

15. Kang, S. -P.; Lee, H. Environ. Sci.Technol. 2000, 34, 4397.

16. Miller, B.; Strong, E. R. Am. Gas. Assn. Monthly 1946, 28, 63

17. Sloan, E. D.; Koh, C. A. Clathrate hydrates of natural gases, $3^{\text {rd }}$ ed, 2007.

18. Belosludov, V. R.; Lavrentiev, M. Y.; Dyadin, Y. A. J. Inclus. Phenom. Mol. 1991, 10, 399.

19. Jeffry, G. A.; McMullan, R. K. Prog. Inorg. Chem. 1967, 8, 43.

20. Mark, T.C.; McMullan, R. K. J. Chem. Phys. 1965, 42, 2732.

21. Ripmeester, J. A.; Tse, J.S.; Ratcliffe, C.I.; Powell, B.M. Nature 1987, 352, 135.

22. McMullan, R. K.; Jeffry, G. A. J. Chem. Phys. 1965, 42, 2725.

23. Berendsen, H. J. C.; Postma, J. P. M.; Van Gunsteren, W. F.; Hermans, J. Interaction Models for Water in Relation to Protein Hydration. Reidel, Dordrecht, 1981.

24. Sparks, K. A. Configurational properties of water clathrates through molecular simulation, PhD Thesis, Massachusetts Institute of Technology, 1991.

25. Bernal, j.D.; Fowler, R. H. J.Chem.Phys. 1993, 1, 515.

26. Rahman, A.; Stillinger, F. H. J. Chem. Phys. 1972, 57, 4009.

27. Barrer, R. M.; Stuart, W. I. Proc. Roy. Soc. Lond. A. 1957, 243, 172.

28. van der Waals, J.H.; Platteeuw, J.C. Adv. Chem. Phys. 1959, $2,1$.

29. McKoy, V.; Sinanoglu, O. J.Chem.Phys. 1963, 38, 2946.

30. Marshall, D. R.; Saito, S.; Kobayaski, R. AIChE J. 1964, 10, 723.

31. Kobayashi, R.; Katz, D. L. J. Petrol Technol. 1949, 1, 66.

32. Parrish, W. R.; Prausnitz, J. M. Ind. Eng.Chem.proc. Des.Dev. 1972, 11, 26.

33. Anderson, F.E.; Prausnitz, J.M. AIChE J. 1986, 32, 1321. 
34. Englezos, P.; Bishnoi, P. R. AIChE J. 1988, 34, 1718.

35. John, V.T.; Holder, G.D. J. Phys.Chem. 1981, 85, 1811.

36. John, V.T.; Holder, G.D. J. Phys.Chem. 1982, 86, 455.

37. Rodger, P. M. J. Phys. Chem. 1989, 93, 6850.

38. Avlonitis, D.; Danesh, A,;

39. Avlonitis, D.; Todd, A. C.; Danesh, A. A

40. Tester, J. W.; Bivins, R. L.; Herrick, C. C. AIChE J. 1972, 31, 252.

41. Metropolis, N.; Rosenbulth, A. W.; Rosenbluth, M. N.; Teller, A. H.; Teller, E. J.Chem. phys. 1953, 21, 1087.

42. Natarajan, V.; Raj, B. P. Ind.Eng.Chem.Res. 1995, 34, 1494

43. Bazant, Z. M.; Trout, L. B. Physica A 2001, 300, 139.

44. Sparks, K. A.; Tester, J. W. J. Phys. Chem. 1992, 96, 11022.

45. Cao, Z. T.; Tester, J. W.; Sparks, K. A.; Trout, B. L. J. Phys. Chem. B. 2001, 105, 10950.

46. Cao, Z. T.; Tester, J. W.; Trout, B. L. J. Phys. Chem. B. 2002, 106, 7681.

47. Cao, Z. T.; Tester, J. W.; Trout, B. L. J. Phys. Chem. 2001, 115, 2550.

48. Klauda, J. B.; Sandler, S. I. J. Phys. Chem. B. 2002, 106, 5722.

49. Anderson, B. J.; Tester, J. W.; Trout, B. L. J. Phys. Chem. B. 2004, 108, 18705.

50. Goldman, S. Can. J. Chem. 1974, 52, 1668.

51. Peterson, K. I.; Klemperer, W. J. Chem. Phys. 1984, 80, 2439.

52. Mehler, E. L. J. Chem. Phys. 1981, 74, 6298.

53. Block P. A., Marshall M. D., Pedersen L. G., and Miller R. E. J. Chem. Phys. 1992, 96, 7321.

54. Makarewicz J., Ha T.-K., and Bauder A. J. Chem. Phys. 1993, 99, 3694.

55. Kieninger M. and Ventura O. N. (1997) J. of Molecular Structure: THEOCHEM 1997, 390, 157.

56. Sun, R.; Duan, Z. Geochimica et Cosmochimica Acta. 2005, 69, 4411.

57. Sadlej, J.; Makarewicz, J.; Chalasinski, G. J. Chem. Phys. 1998, 109, 3919.

58. Kaluda, J. B.; Sandler, S. I. Ind. Eng. Chem. Res. 2000, 39, 3377

59. Danten, Y.; Tassaing, T.; Besnard, M. J. Phys. Chem. A 2005, 109, 3250.

60. Anderson, B. J.; Bazat, M. Z.; Tester, J. W.; Trout, B. L. J. Phys. Chem. B. 2005, 109, 8153.

61. Holder, G.D.; Zetts, P. S.; Pradhan, N. Reviews in Chemical Engineering 1988, 5, 1.

62. Bozzo, A. T.; Chen, H.-S.; Kass. J. R.; Barduhn, A. J. Desalination 1975, 16, 303.

63. Davidson, D. W.; Handa, Y. P.; Ripmeester, J. A. J. Phys. Chem. 1986, 90, 6549.

64. Sum, A. K.; Burruss, R. C.; Sloan, D. E. J. Phys.Chem. B 1997, 101, 7371.

65. Ikeda, T.; Yamamuro, Matsuo, T.; Mori, K.; Torii, S.; Kamiyama,T.; Izumi, F.; Ikeda, S.; Mae, S. J. Phys. Chem. Solids 1999, 60, 1527.

66. Udachin, K. A.; Ratcliffe, C. I.; Ripmeester, J. A. J. Phys.Chem. B 2001, 105, 4200.

67. Henning, R. W.; Schultz. A. J.; Thieu, V.; Halpern, Y. J. Phys.Chem. A 2000, 104, 5066.

68. Ripmeester, J. A.; Ratcliffe, C. I. Energy Fuels 1998, 12, 197.

69. Ohgaki, K.; Takano, K.; Sangawa, H.; Matsubara, T.; Nakano, S. J. Chem. Eng. Jpn. 1996, $29,478$.

70. Hester, K.C.; Huo, Z.; Ballard, A. L.; Koh, C.A. Miller, K. T.; Sloan, E. D. J. Phys. Chem. B 2007, 111, 8830 .

71. Hwang, M. J.; Holder, G. D.; Zele, S. R. Fluid Phase Equilibr. 1993, 83, 437. 
72. Zele, S. R.; Lee, S.-Y.; Holder, G.D. J. Phys. Chem. B 1999, 103, 10250.

73. Lee, S. -Y.; Holder, G. D. AIChE J 2002, 48, 161. 


\section{Theoretical Model for Gas Hydrates}

\subsection{Statistical Thermodynamic model}

Gas hydrates consists of two types of molecules, water and typically a non polar gas, which are not chemically bonded. A simple gas hydrate can be considered as a two component system consisting of a guest molecule and water molecules. The temperature and pressure conditions determine in what phases the guest molecule and the host molecule will exist. From the phase diagram as shown in Figure 1.1 for $\mathrm{CH}_{4}$ and $\mathrm{CO}_{2}$ hydrate, we can say that the hydrate formation is favored at low temperature and high pressure. The equilibrium vapor pressure, often referred to as the dissociation pressure, is commonly measured as a function of temperature for various three-phase, monovariant systems. Gas hydrate thermodynamics make it possible to predict the temperature and pressures conditions at which hydrate form or decompose.

The criterion for the phase equilibrium is the equality of chemical potentials $(\mu)$ of each component in the coexisting phases. At equilibrium,

$$
\mu_{w}{ }^{H}(T, P)=\mu_{w}{ }^{L, \alpha}(T, P)
$$

where $\mu_{w}{ }^{H}(T, P)$ is the chemical potential of water in the hydrate phase, and $\mu_{w}{ }^{L, \alpha}(T, P)$ is the chemical potential of water in the water rich $(L)$ or ice phase $(\alpha)$ at temperature, $T$, and pressure, $P$. The water rich liquid or ice phase is dependent on whether the temperature is 
above $273.15 \mathrm{~K}$ or not. Using $\mu_{w}{ }^{\beta}$, the chemical potential of hypothetical empty hydrate lattice, the condition for equilibrium can be written as in Equation 2.2.

$$
\Delta \mu_{w}^{\beta-H}=\Delta \mu_{w}^{\beta-L, \alpha}
$$

where

$$
\begin{gathered}
\Delta \mu_{w}^{\beta-H}=\mu_{\beta}^{\beta}-\mu_{w}{ }^{H} \\
\Delta \mu_{w}^{\beta-L, \alpha}=\mu_{w}^{\beta}-\mu_{w}{ }^{L, \alpha}
\end{gathered}
$$

The initial statistical thermodynamics model to determine the gas hydrates properties was suggested by Barrer and Straut ${ }^{1}$. With the knowledge of the crystal structures of hydrates, van der Waals and Platteeuw ${ }^{2}$ proposed a basic model based on classical statistical thermodynamics corresponding to the three dimensional generalization of ideal localized adsorption, derived the grand canonical partition function for water, with the following assumptions.

1) Each cavity can contain at most one gas molecule.

2) The interaction between a gas and water molecule can be described by a pair potential functions, and the cavity can be treated as perfectly spherical.

3) The free energy contribution of the water molecules is independent of the mode of dissolved gases (cage distortions are neglected)

4) There is no interaction between the gas molecules in different cavities and the guest molecule interacts only with the nearest neighbor water molecules (guest-guest interactions are neglected).

The chemical potential difference between the empty lattice and fully filled hydrate lattice can be expressed as 


$$
\Delta \mu_{w}{ }^{\beta-H}=-R T \sum_{i=1}^{n} v_{i} \ln \left(1-\sum_{j} \theta_{j i}\right)
$$

where $v_{i}$ is the number of $i$-types cavities per water molecule, $R$ is the gas constant and $T$ is the temperature. $\theta_{j i}$ is the fractional occupancy of $i$-type cavities with $j$-type guest molecules. $n$ is the number of cavities and is equal to 2 for sI and sII, $n=3$ for structure H. From the Equation 2.3 , the chemical potential of the hydrate is reduced by the potential interactions of the guest and the host water molecules. The greater the fraction of cavities occupied lesser is the chemical potential of the hydrate and water. Clathrate hydrates are non stoichiometric compounds, therefore the cage occupancy, $\theta_{j i}$, is $\leq 1$ and also a function of equilibrium conditions. Mathematically, the cage occupancy, $\theta_{j i}$, follows the Langmuir isotherm and expressed in terms of Langmuir constant as

$$
\theta_{j i}=\frac{C_{j i} f_{j}}{1+\sum_{i} C_{j i} f_{j}}
$$

where $f_{i}$ is the fugacity of gas component $i$, calculated using a PVTN equation of state, after the Peng-Robinson equation of $\operatorname{state}^{3} . C_{j i}$ is the temperature-dependent Langmuir constant for species $i$ in cavity $j$ defined as

$$
C_{j i}=\frac{Z_{j i}}{k T}=\frac{1}{8 \pi^{2} k T} \int_{V} \exp \left(-\frac{\Phi(r, \theta, \phi, \alpha, \beta, \gamma)}{k T}\right) r^{2} \sin \theta d r d \theta d \phi d \alpha d \beta d \gamma
$$

where $Z_{j i}$ is the configurational integral and $\Phi$ is the interaction potential between the guest molecule and the host molecules surrounding it. The Langmuir constant is actually the description of the affinity of the empty cavity for a molecule to occupy this cavity, higher values of the Langmuir constant indicate that a guest molecule is more likely to be encaged. 
Langmuir constant will approach to zero when the guest molecule is small compared to the cavity.

For the structure I hydrate, the unit cell has 46 water molecules with 2 small cavities and 6 large cavities. The number of small cavities per water molecule, $v_{1}$, is equal to $1 / 23$, the number of large cages, $v_{2}$, is equal to $3 / 23$, the complete expression for a pure component structure I water clathrates system is

$$
\frac{\Delta \mu_{w}^{\beta-H}}{k T}=\frac{1}{23} \ln \left(1+C_{j 1} f_{j}\right)+\frac{3}{23} \ln \left(1+C_{j 2} f_{j}\right)
$$

The structure II hydrate unit cell has 136 water molecules with 16 small cavities and 8 large cavities. The ratio of small cavities to water molecules, $v_{1}$, equals $2 / 17$, and the number of large cages, $v_{2}$, is equal to $1 / 17$. The complete expression for a pure component structure II water clathrates system is

$$
\frac{\Delta \mu_{w}^{\beta-H}}{k T}=\frac{2}{17} \ln \left(1+C_{j 1} f_{j}\right)+\frac{1}{17} \ln \left(1+C_{j 2} f_{j}\right)
$$

The chemical potential difference, $\Delta \mu_{w}$, between the hypothetical empty hydrate lattice and water in the hydrate phase is given by Holder et $\mathrm{al}^{4}$ as

$$
\frac{\Delta \mu_{w}{ }^{\beta-L, \alpha}(T, P)}{k T}=\frac{\Delta \mu_{w}^{\beta-L, \alpha}\left(T_{o}, 0\right)}{k T}-\int_{T_{o}}^{T} \frac{\Delta h_{w}^{\beta-L, \alpha}}{k T^{2}} d T+\int_{o}^{P} \frac{\Delta V_{w}^{\beta-L, \alpha}}{k T} d P-\ln a_{w}
$$

where $\Delta \mu_{w}{ }^{\beta-L, \alpha}\left(T_{o}, 0\right)$ is the reference chemical potential difference at the reference temperature, $T_{o}$, and zero pressure. The reference temperature, $T_{o}$, is the ice point temperature. In case of methane hydrate the ice point temperature $T_{o}=273.15 \mathrm{~K}$ and in case of carbon 
dioxide hydrate, $T_{o}$ is $271.75 \mathrm{~K}$. The depression in the ice point temperature for $\mathrm{CO}_{2}$ hydrate is due to the high solubility of carbon dioxide in water. The second term on the left of Equation 2.8 gives the temperature dependence at constant pressure. The third term corrects the pressure to the final equilibrium pressure and the last term corrects the chemical potential from pure water phase to water rich solution. The temperature dependent enthalpy difference is given by Equation 2.9.

$$
\Delta h_{w}^{\beta-L, \alpha}=\Delta h_{w}^{o}\left(T_{o}\right)+\int_{T_{o}}^{T} \Delta C_{P} d T
$$

where the $\Delta h_{w}{ }^{o}\left(T_{o}\right)$ is the reference enthalpy difference between the empty hydrate lattice and the pure water phase at reference temperature $T_{o}$. The heat capacity difference between the empty hydrate lattice and the pure water phase, $\Delta C_{P}$, is also temperature dependent and it is approximated by the following expression

$$
\Delta C_{P}=\Delta C_{P}^{\mathrm{o}}\left(T_{o}\right)+b\left(T-T_{o}\right)
$$

where $\Delta C_{P}{ }^{\circ}\left(T_{o}\right)$ is the reference heat capacity difference at the reference temperature $T_{o}$. The constant, $b$, represents the dependence of heat capacity on the temperature. Two different expressions must be used for the water in liquid phase and in solid phase. The volume difference, $\Delta V_{w}{ }^{\beta-L, \alpha}$, is assumed to be constant. The last term in the Equation 2.8 is activity of water, $a_{w}$, is defined as

$$
a_{w}=\frac{f_{w}{ }^{L}}{f_{w}}
$$

where $f_{w}{ }^{L}$ is the fugacity of water in the water rich aqueous phase and $f_{w}$ is the water fugacity at the reference state, the pure water phase. The reference parameters found in the literature for 
structure I are shown in the Table 2.1 and the thermodynamic reference properties used in this work are given in Table 2.2.

Table 2.1 Thermodynamics reference properties for structure I

\begin{tabular}{|c|c|c|}
\hline$\Delta \mu_{w}^{\beta-L, \alpha}\left(T_{o}, 0\right)$ & $\Delta \mathrm{H}_{w}^{\beta-L, \alpha}\left(T_{o}, 0\right)$ & Source $^{\mathrm{a}}$ \\
\hline 699 & 0 & van der Waals and Platteeuw (1959) \\
\hline 1255.2 & 753 & Child (1964) \\
\hline 1264 & 1150 & Parrish and Prausnitz (1972) \\
\hline 1155 & 381 & Holder (1976) \\
\hline 1297 & 1389 & Dharmawardhana, Parrish and Sloan \\
\hline 1299 & 1861 & Holder, Malekar and Sloan (1984) \\
\hline 1120 & 931 & John, Papadopoulos and Holder (1985) \\
\hline 1287 & 931 & Handa and Tse (1986) \\
\hline 1287 & - & Davidson, Handa and Ripmeester (1986) \\
\hline 1236 & 1703 & Cao, Tester and Trout (2002) \\
\hline 1203 & 1170 & Anderson, Tester, Trout (2004) \\
\hline 1202 & 1300 & Sun and Duan (2005) \\
\hline
\end{tabular}

${ }^{\mathrm{a}} \operatorname{Ref} 2,5-13,30$ 
Table 2. 2 Thermodynamic reference properties for structure I

\begin{tabular}{|c|c|c|}
\hline & Structure I & Reference \\
\hline$\Delta \mu_{w}{ }^{o}(\mathrm{~J} / \mathrm{mol})$ & 1217 & Parameters for $\mathrm{CO}_{2}$ \\
hydrate (This work)
\end{tabular}

\subsection{Configurational partition function}

The most important term in the van der Waals and Platteeuw ${ }^{2}$ model is the Langmuir constant, which is the key to predict the cage occupancies and phase equilibrium of gas hydrate. The Langmuir constant depends on the guest-host interactions. In the thermodynamic model, all parameters except for the Langmuir constant can be determined from either experimental data or in the case of fugacity from an equation of state. For a guest molecule $j$ in a cavity of type $i, C_{J i}$ is directly related to the six dimensional configurational integral over a system volume $V$ defined by

$$
Z_{j i}=\frac{1}{8 \pi^{2}} \int_{V} \exp \left(-\frac{\Phi(r, \theta, \phi, \alpha, \beta, \gamma)}{k T}\right) r^{2} \sin \theta d r d \theta d \phi d \alpha d \beta d \gamma
$$


where $Z_{j i}$ is the configurational integral, which depends on the interaction potential, $\Phi$, between the guest molecule $j$ in the cavity $i$ and all the host molecules surrounding it. The interaction potential is a function of the position and orientation of the guest in the cavity and is given by the spherical coordinates $r, \theta, \phi$ and the Euler angles $\alpha, \beta$ and $\gamma$ which describe the orientation of the guest. The factor of $8 \pi^{2}$ is the normalizing constant coming from the volumetric integration. The total interaction potential $\Phi=\sum_{\mathrm{ij}} \Phi_{\mathrm{ij}}$ between the guest and all the host water molecules must be represented properly to calculate the configurational integral accurately. The original work by van der Waals and Platteuw used the Lennard Jones (L-J) 612 pair potential. McKoy and Sinangolu ${ }^{16}$ suggested that the Kihara potential is better than the Lennard Jones potential. The potential parameters were obtained by empirically fitting to the experimental hydrate dissociation data. However, these empirically-fitted potential parameters are aphysical and don't match those determined using gas phase experimental data ${ }^{10,17,18}$.

\subsubsection{LJD approximation}

The asymmetry of the host cavities and an asymmetric guest molecule makes the configurational partition function to be a six dimensional integral (Equation 2.12). The analytical evaluation of this six dimensional integral is intractable, so several approximations have been applied. Most commonly, the Lennard-Jones and Devonshire (LJD) cell model is adopted for the quantitative evaluation of the configurational integral. In this, the host water molecules are assumed to be uniformly distributed on a spherical surface corresponding to an average cavity radius. The guest molecule is also usually assumed to be spherically symmetric ( $\Phi$ independent of $\alpha, \beta, \gamma$ ). In this case, the smooth cell potential is independent of angular 
coordinates $(\theta$ and $\phi)$ and depends on the radial distance, $r$, only ${ }^{3}$. This simplifies the six dimensional configurational integral to one dimensional integral. The smoothed cell Langmuir constant, $C_{j i}{ }^{*}$, is expressed as

$$
C_{j i}^{*}=\frac{4 \pi}{k T} \int_{0}^{R} \exp \left\langle-\frac{W(r)}{k T}\right\rangle r^{2} d r
$$

The angle averaged spherically symmetric cell potential is determined from

$$
\left\langle\frac{W(r)}{k T}\right\rangle=\frac{1}{4 \pi} \int_{0}^{2 \pi} \int_{0}^{\pi} \Phi_{j i}(r, \theta, \phi) \sin \theta d \theta d \phi
$$

Using the Kihara potential as shown in Equation 2.25 for the guest- host interactions, the spherically averaged cell potential obtained is

$$
\left\langle\frac{W(r)}{k T}\right\rangle=2 z \varepsilon\left[\frac{\sigma^{12}}{R^{11} r}\left(\delta^{10}+\frac{a}{R} \delta^{11}\right)-\frac{\sigma^{6}}{R^{5} r}\left(\delta^{4}+\frac{a}{R} \delta^{5}\right)\right]
$$

where

$$
\delta^{N}=\frac{1}{N}\left[\left(1-\frac{r}{R}-\frac{a}{R}\right)^{-N}-\left(1+\frac{r}{R}-\frac{a}{R}\right)^{-N}\right]
$$

where $N$ is 4, 5, 10, 11 indicated in Equation 2.15, $z$ is the coordination number of the cavity, $R$ is the effective cavity radius, $r$ is the distance of the guest molecule from the cavity center, $a$ is the core radius of interaction, $\sigma$ is the distance between molecular cores at which there is no interaction, and $\varepsilon$ is the depth of the intermolecular potential well. 


\subsubsection{Monte Carlo method}

Tester et al. ${ }^{19}$ has accounted the asymmetries of the host molecules and guest molecule in the configurational partition function and evaluated by using a Metropolis sampling Monte Carlo procedure ${ }^{20}$. These asymmetries made the configurational integral to a six dimensional integral. The Monte Carlo (MC) method is a stochastic method using a random number for the arrangements of molecules under a law of probability. The transitions between different states or configurations are achieved by 1) generating a random trail configuration, 2) an acceptance criteria was evaluated by calculating the change in energy and other properties in the trial configurations and 3) comparing the acceptance criterion to a random number and either accepting or rejecting it in the trial configuration. In this, the acceptance or rejection of the step is dependent on the basis of the Metropolis et $\mathrm{al}^{20}$ technique.

In evaluating the configurational integral by Monte Carol method, the Langmuir constant is approximated as the product of averaged energy and volume and is expressed by Tester et $\mathrm{al}^{19}$ as

$$
Z_{j i}=\int_{V} e^{-\frac{U}{k T}} d V \approx\left\langle e^{\left.-\frac{U}{k T}\right\rangle} V \approx e^{\left(-\frac{\langle U\rangle}{k T}\right)} V_{c e l l}\right.
$$

where $\langle U\rangle$ is the ensemble average of the potential energy obtained by MC sampling and $V_{\text {cell }}$ is the effective "free volume" available to the guest molecule within the clathrate cage. The ensemble averages $\langle U\rangle$ are approximated by

$$
\langle U\rangle \approx \frac{1}{N} \sum_{k=1}^{N} U
$$

where $\mathrm{N}$ is the number of random moves made with the guest molecules. $U$ is the interaction energy calculated and accepted at move number $k$. The potential energy $U$ at a point $k$ is 
calculated as the pair wise between the guest molecule and host molecules is given as

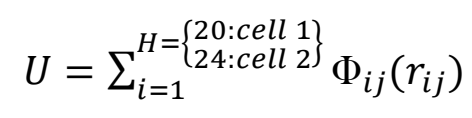

The interaction potential $\Phi_{i j}$ between the guest and the host water molecules is represented by Lennard-Jones (L-J) 6-12 potential for symmetric guest and Kihara potential for polyatomic guests. The details of theses potentials are discussed in Section 2.3. The Lennard-Jones parameters for the argon were adjusted to constrain the predicted dissociation pressure to match the experimental dissociation pressure of the argon-water clathrate. Using the Berthelot geometric mean approximation for $\varepsilon$, and the hard sphere approximation for $\sigma$, the LennardJones parameter for water, $\varepsilon_{\mathrm{H}_{2}} \mathrm{O}$, was calculated. These "adjusted" parameters were then used to predict the dissociation pressures of other gas hydrate systems. Natrajan and Bishoni ${ }^{21}$ computed the Langmuir constant from Multi dimensional integral methods and by Metropolis MC method. The MC method gives erroneous computed Langmuir constants owing to the errors in calculating the energies and the free volumes in the Equation 2.17. The free volume, $V_{\text {cell }}$, is not just the volume of the guest; this volume is estimated in terms of the region in which moves are accepted. The calculation of this free volume is difficult to calculate with sufficient accuracy and eventually give rise to the errors in Langmuir Constant.

The equation given by Sparks et al. ${ }^{22}$ for calculating the Langmuir constant for asymmetric guest molecules by applying simple Monte Carlo integration to the configuration integral is

$$
Z_{j i} \cong \frac{\pi^{3} R}{N} \sum_{k=1}^{N} \exp \left(-\frac{\Phi(r, \theta, \phi, \alpha, \beta, \gamma)}{k T}\right) r_{k}^{2} \sin \theta_{k} \sin \beta_{k}
$$




\subsubsection{Integration methods}

The total interactions between the guest and the host water molecules must be represented properly in order to calculate the configurational integral accurately. Sparks et al ${ }^{22}$ computed the the guest-host configurational integral accounting the asymmetry of the cages by simple Monte Carlo integration, the composite trapezoidal rule, and Gauss-Legendre quadrature integration techniques. The MC method is not well suited for efficiently estimating the potential energy profiles in the host lattice cavities, which gives errors in the Langmuir constant calculations. Considering the geometric complexities of water clathrates system, they found that the multi-interval 10 point Gauss-Legendre quadrature formula is much more accurate than the composite trapezoidal rule. The 10 point Gauss-Legendre quadrature formula ${ }^{23}$ :

$$
\int_{a}^{b} f(x) d x=\int_{a}^{b / n} f(x) d x+\int_{b / n}^{2 b / n} f(x) d x+\cdots+\int_{(n-1) b / n}^{b} f(x) d x
$$

\subsection{Intermolecular potential function}

The intermolecular potentials between the guest and the host water molecules must be represented properly for the accurate evaluation of the Langmuir constant as shown in Equation 2.5, which is the key term in the van der Waals and Platteeuw model. The total interaction potential between each guest $(j)$ molecule and all the host water molecules is modeled as a pair wise additive

$$
\Phi(r, \theta, \phi, \alpha, \beta, \gamma)=\sum_{i=1}^{N} \Phi_{\mathrm{ij}}(r, \theta, \phi, \alpha, \beta, \gamma)
$$


where the sum is over all $N$ interacting host water molecules.

van der Waals and Platteeuw in their original work modeled the guest host intermolecular potential using Lennard- Jones 6-12 interaction potential. The L-J 612 model is illustrated in the Figure 2.1

Lennard-Jones 6-12 potential is

$$
\Phi(r)=4 \varepsilon\left[\left(\frac{\sigma}{r}\right)^{12}-\left(\frac{\sigma}{r}\right)^{6}\right]
$$

where $r$ is the distance between molecular centers, $\sigma$ is the collision diameter, and $\varepsilon$ is the characteristic energy. Using the L-J 6-12 potential along with the LJD approximation predicted equilibrium dissociation pressure very well for the noble gas hydrates like $\mathrm{Ar}, \mathrm{Kr}$ and $\mathrm{Xe}$., but large discrepancies exists for the more complex and large guest molecule like ethane and cyclopropane.

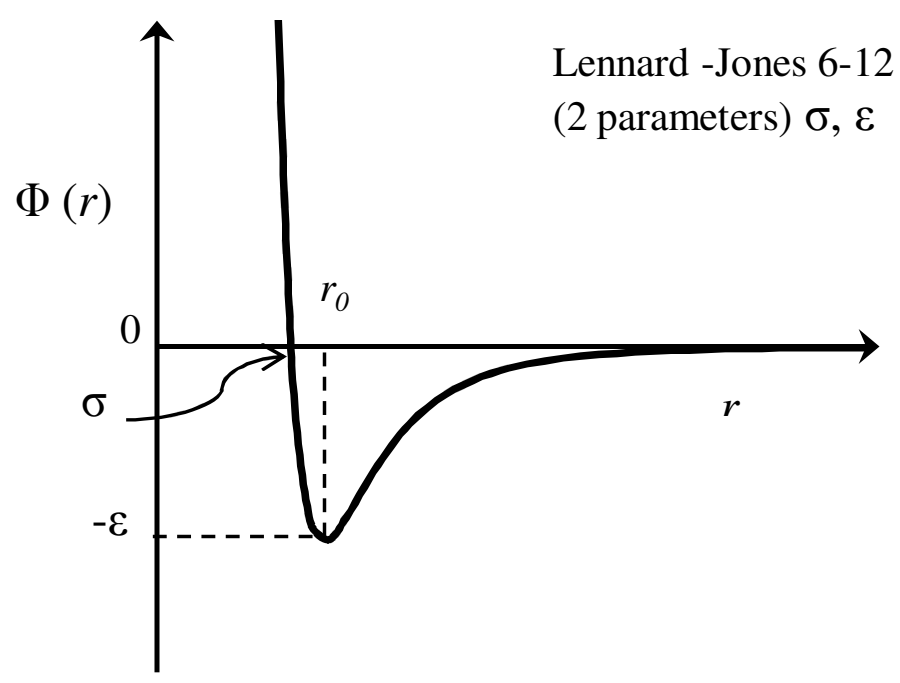

Figure 2.1 Lennard - Jones 6-12 potential parameter 
McKoy and Sinangolu ${ }^{16}$ suggested that the Kihara Potential with the LJD spherical cell approximation can fit the experimental data better than the L-J 6-12 potential for larger polyatomic and rod like molecules. This is because the Kihara potential has three adjustable parameters compared to that L-J 6-12 which has two adjustable parameters to fit the experimental data. The Kihara 3 parameter potential form is illustrated in Figure 2.2. The Kihara potential has been extensively used in modeling the guest host intermolecular potential in many clathrate hydrate systems.

The Kihara Potential:

$$
\begin{array}{cc}
\Phi(r)=\infty & r \leq 2 a \\
\Phi(r)=4 \varepsilon\left[\left(\frac{\sigma-2 a}{r-2 a}\right)^{12}-\left(\frac{\sigma-2 a}{r-2 a}\right)^{6}\right] & r \geq 2 a
\end{array}
$$

where $2 a$ is the molecular core diameter, $\sigma$ is the collision diameter, and $\varepsilon$ is the characteristic energy. The spherically averaged LJD form of Kihara potential is shown in Equations 2.15, 2.16 .

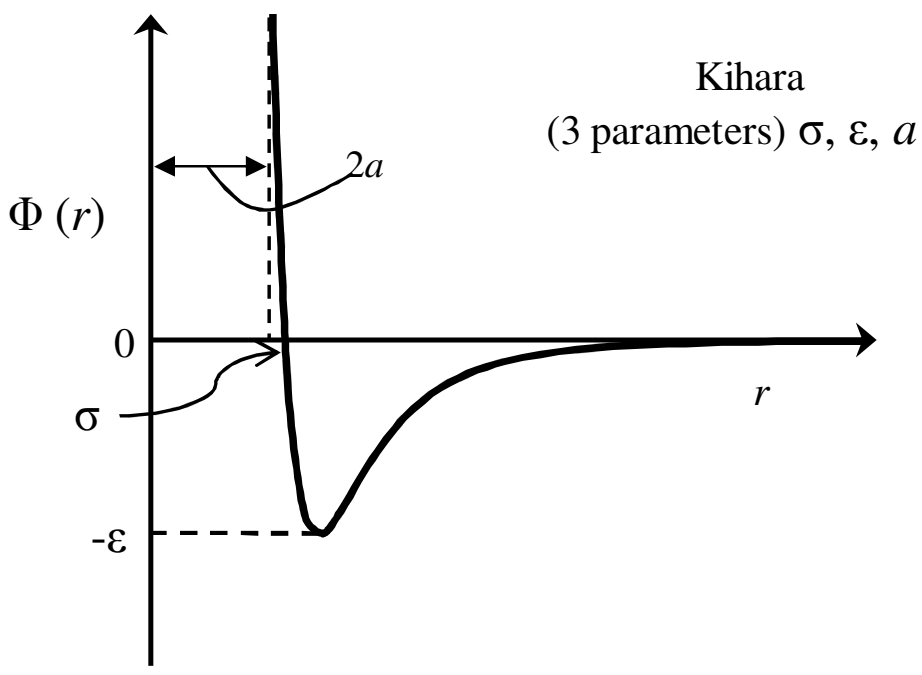

Figure 2.2 Kihara intermolecular potential 
The parameters of the Kihara potential and the L-J 6-12 potentials are generally found by fitting to the experimental dissociation pressure data. These potentials lack a molecular basis, and must be determined ad hoc for each hydrates system. The Kihara potential is only empirically superior because of the three adjustable parameters. The Kihara potential can yield better results than the L-J 6-12 potential. This does not mean that Kihara potential is more realistic, they are only empirically superior because of the three adjustable parameters. Furthermore, in the total interaction potential only the first water shell of water molecules surrounding the guest molecules was considered initially. Sparks et al. ${ }^{24}$ showed that the shell other than the first shell also contribute to the total interaction potential. These empiricallybased potentials do not provide the true nature of the potential of interaction. Alternately, the analytical intermolecular potential functions determined from the first principle, ab initio, quantum mechanical calculations describe more accurately the interactions between the guest and host water molecules and avoids the need to fit potential functions to experimental data ${ }^{25}$.

Cao et al. ${ }^{25,26}$ determined the ab initio potential energy surface for $\mathrm{CH}_{4}-\mathrm{H}_{2} \mathrm{O}$ dimer and applied to predict the phase equilibrium of methane hydrate. They had calculated the ab initio binding energies for 18,000 interactions between methane and single water molecule to sample the potential energy surface accurately. However, they performed spherical averaging on the intermolecular potentials with the Boltzmann averaging algorithm resulting in the loss of the quality of $a b$ initio potential. This averaging result the errors in cage occupancy predictions. Anderson et al. ${ }^{28}$ improved the work of Cao et al. ${ }^{25,26}$ by using the site-site potential model to fit the $a b$ initio potential for $\mathrm{CH}_{4}-\mathrm{H}_{2} \mathrm{O}$. They have also performed $a b$ initio calculations to determine the intermolecular potential energy surface for argon and water system. The pair wise $a b$ initio potentials were modeled using L-J 6-12 potentials and exponential-6 potentials. 
Exponential -6:

$$
\Phi(\mathrm{r})=\frac{\varepsilon}{1-6 / \gamma}\left[\frac{6}{\gamma} \exp \left[\gamma\left(1-\frac{r}{r_{m}}\right)\right]-\left(\frac{r_{m}}{r}\right)^{6}\right]
$$

where $\varepsilon, \gamma$ and $r_{m}$ are model parameters. The radial distance at which the potential is a minimum is given by $r_{m}$ and $\varepsilon$ is the characteristic energy. The exponential- 6 potential form is shown in Figure 2.3

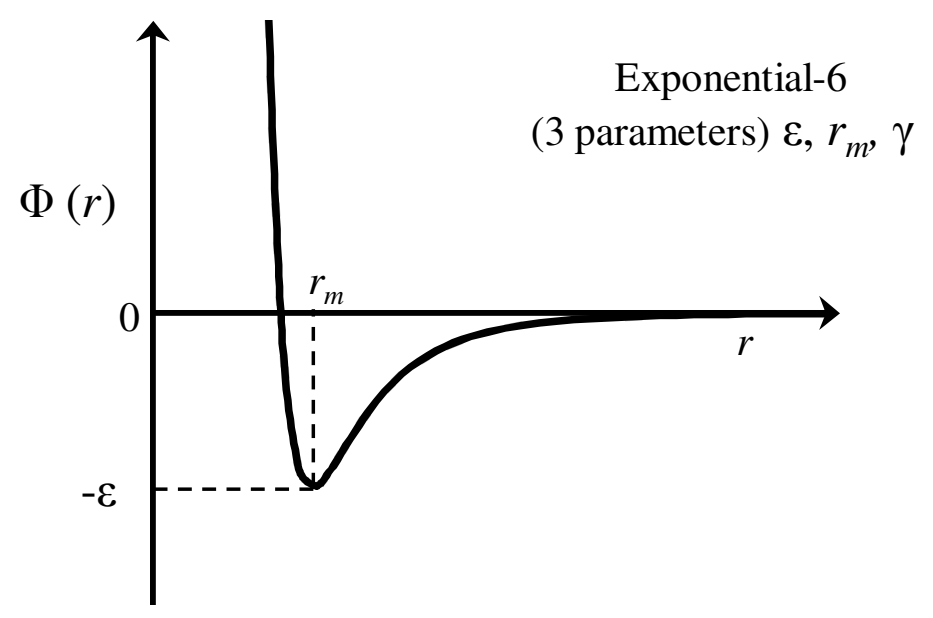

Figure 2.3 Exponential-6 intermolecular potential 


\subsection{Prediction of Hydrate Phase Diagram}

Parrish and Prausnitz ${ }^{6}$ developed an algorithm, for calculating the hydrate formation conditions in gas mixtures. The basic idea of the algorithm is to predict the three-phase hydrate equilibrium through an iterative process at a given temperature until the chemical potential difference calculated from Equations 2.3 and 2.8 are equal with an error criterion. This algorithm is used in our prediction of pure component hydrate phase diagrams with a simplification to eliminate the reference hydrate suggested by Holder et al. ${ }^{4}$ as shown in Equation 2.8. An initial guess for the pressure is estimated from the empirical equation shown in Equation 2.27.

$$
\ln P=A+B+C \ln T
$$

where $A, B$ and $C$ are constants determined from experimental data. The iterative procedure for the prediction of dissociation pressure is as follows ${ }^{6}$ :

1) Initialize all the parameters needed in Equations 2.3 and 2.8 like reference parameters, intermolecular potentials.

2) Read the temperature $T$

3) Give an initial estimate for pressure $P_{o}$ from Equation 2.27, assume Structure I

4) Calculate the Langmuir constant from Equation 2.5

5) Calculate $\Delta \mu_{w}{ }^{\beta-\alpha}\left(T, P_{o}\right)$ from Equation 2.8 and the fugacity is calculated from the equation of state (EOS).

6) Holding $\Delta \mu_{w}{ }^{\beta-\alpha}\left(T, P_{o}\right)$ and the fugacity calculated from EOS to be constant calculate pressure $P_{1}$ from Equation 2.3. 
7) If $P_{1} \neq P_{o}$ repeat with a new pressure from step 2. If $P_{l}=P_{o}$ with an error criteria, then $P_{l}$ is the equilibrium pressure at temperature $T$.

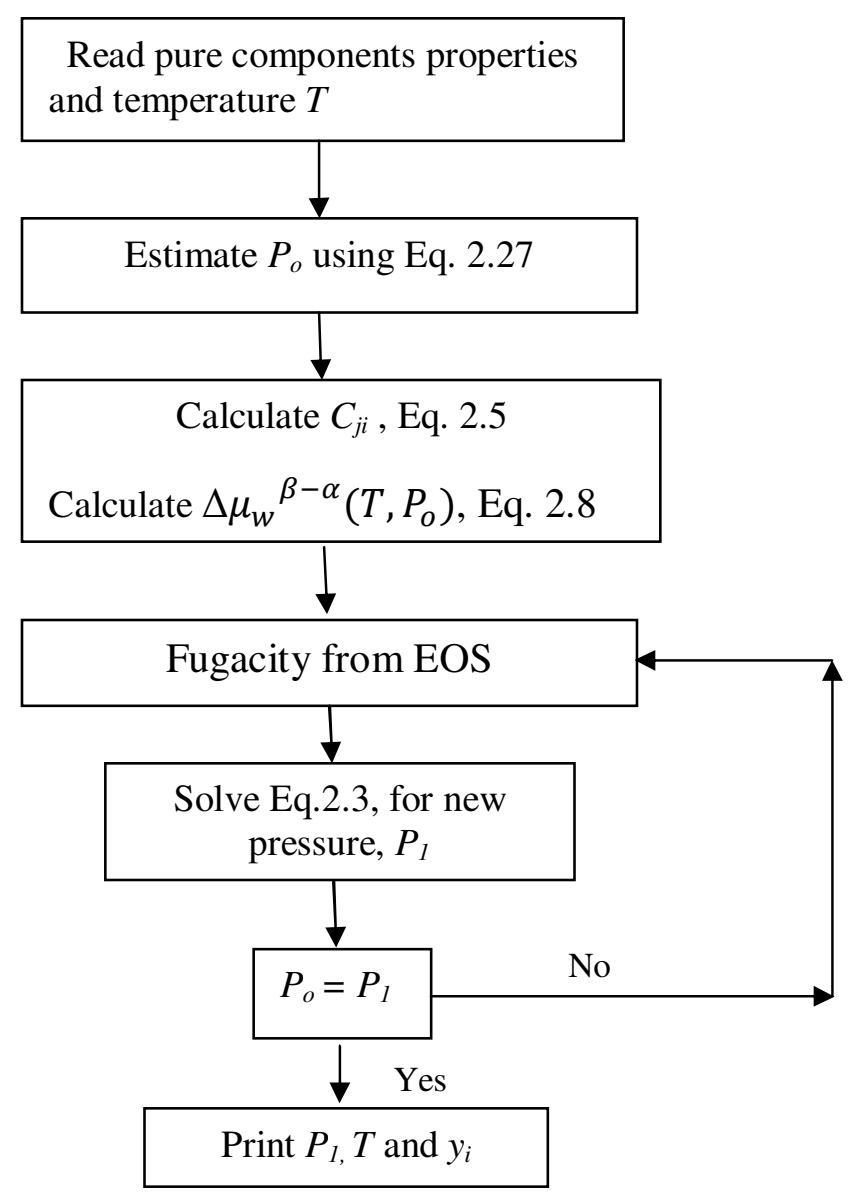

Figure 2.4 Schematic of computer program for calculating equilibrium pressure. 


\subsection{References}

1) Barrer, R. M.; Stuart, W. I. Proc. Roy. Soc. Lond. A. 1957, 243, 172.

2) van der Waals, J.H.; Platteeuw, J.C. Adv. Chem. Phys. 1959, 2, 1.

3) Peng, D.- Y.; Robinson, D. B. Ind. Eng. Chem. Fund. 1976, 15, 59.

4) Holder, G. D.; Corbin, G; Papadopoulos, K. D. Ind. Eng. Chem. Fund. 1980, 19, 282.

5) Child, W.C., Jr. J. Phys. Chem. 1964, 68, 1834.

6) Parrish, W. R.; Prausnitz, J. M. Ind. Eng. Chem. Proc. Des. Dev. 1972, 11, 26.

7) Holder, G.D.; Katz, D.L.; Hand, J. H. AAPG Bulletin- American Association of Petroleum Geologists 1976, 60, 981.

8) Dharmawardhana, P. B.; Parrish, W.R.; Sloan, E.D. Ind. Eng. Chem. Fund. 1980, 19, 410.

9) Holder, G. D.; Malekar, S. T.; Sloan, E.D. Ind. Eng. Chem. Fund. 1984, 23, 123.

10) John, V. T.; Papadopoulos, K. D.; Holder, G. D. AIChE J. 1985, 31, 252.

11) Handa, Y. P.; Tse, J.S. J. Phys. Chem. 1986, 90, 5917.

12) Davidson, D.W.; Handa, Y. P.; Ripmeester, J. A. J. Phys. Chem. 1986, 90, 6549.

13) Cao, Z. T.; Tester, J. W.; Trout, B. L. J. Phys. Chem. B. 2002, 106, 7681.

14) Anderson, B. J.; Tester, J. W.; Trout, B. L. J. Phys. Chem. B. 2004, 108, 18705.

15) Stackelberg, M. v.; Müller, H.R. Zeitschrift für Electrochemie 1954, 96 , 11022.

16) McKoy, V.; Sinanoglu, O. J.Chem.Phys. 1963, 38, 2946.

17) Sloan, E. D.; Koh, C. A. Clathrate hydrates of natural gases, $3^{\text {rd }}$ ed, 2007.

18) John, V.T.; Holder, G.D. J. Phys.Chem. 1985, 89, 3279.

19) Tester, J. W.; Bivins, R. L.; Herrick, C. C. AIChE J. 1972, 31, 252.

20) Metropolis, N.; Rosenbulth, A. W.; Rosenbluth, M. N.; Teller, A. H.; Teller, E. J.Chem. phys. 1953, 21, 1087.

21) Natrajan, V.; Bishoni, R.P. Ind. Eng. Chem. Res. 1995, 34, 1494.

22) Sparks, K.A.; Tester, J.W.; Cao, Z.; Trout, L.B. J. Chem. Phys. B 1999, 103,6300

23) Carnahan, B.; Luther, H. A.; Wilkes, J. O. Applied Numerical Methods; Wiley: New York, 1969.

24) Sparks, K. A.; Tester, J. W. J. Phys. Chem. 1992, 96, 11022.

25) Cao, Z. T.; Tester, J. W.; Sparks, K. A.; Trout, B. L. J. Phys. Chem. B. 2001, 105, 10950.

26) Cao, Z. T.; Tester, J. W.; Trout, B. L. J. Phys. Chem. 2001, 115, 2550.

27) Klauda, J. B.; Sandler, S. I. J. Phys. Chem. B. 2002, 106, 5722.

28) Anderson, B. J.; Tester, J. W.; Trout, B. L. J. Phys. Chem. B. 2004, 108, 18705.

29) Cao, Z. T.; Tester, J. W.; Trout, B. L. J. Phys. Chem. B. 2002, 106, 7681.

30) Sun, R.; Duan, Z. Geochimica et Cosmochimica Acta. 2005, 69, 4411. 


\section{Ab Initio Intermolecular Potentials for Predicting Cage Occupancy and Phase Equilibrium for $\mathrm{CO}_{2}$ Hydrate}

\subsection{Introduction to $a b$ initio calculations}

The intermolecular potentials between the guest and the host water molecules must be represented properly in order to predict the cage occupancies and to accurately model hydrate equilibrium temperatures and pressures. Most of the early methods empirically fit potential ${ }^{1}$ parameters to hydrate equilibrium pressures using the thermodynamic model developed by van

der Waals and Platteeuw ${ }^{17}$. The potentials obtained work well in the regime of the fitted experimental data range and fail when extended outside the regime. One of the problems with this approach is that there are potentially more than one set of potential parameters that can give accurate equilibrium pressures over a range of conditions ${ }^{1}$, and the guest-host potential energy surface (PES) will differ without a unique set of potential parameters. Unfortunately, current experimental techniques are unable to provide directly measured interaction potentials between $\mathrm{CO}_{2}$ and water. An ab initio quantum mechanical calculation can be used to obtain the intermolecular potentials which forefend the need to fit the potential functions to experimental data.

An ab initio quantum mechanical calculation provides an independent method to directly obtain intermolecular potentials which can be used in gas hydrate modeling. The exact value of the system energy and other properties can be obtained by solving the timeindependent Schrödinger equation described below,

$$
H \Psi=E \Psi
$$


where $H$ is the Hamiltonian operator for the system of nuclei and electrons, $E$ is the energy of the system, and $\Psi$ is the electron wave function. For any but the smallest system, however, exact solutions to the Schrödinger equation are not computationally practical. Therefore, a great number of approximate methods strive to achieve the best trade-off between accuracy and computational cost. The ab initio methods, which do not include any empirical or semiempirical parameters in their equations, are derived directly from theoretical principles, with no inclusion of experimental data. Accuracy can always be improved with greater computational cost and with current computer speed and memory and along with the quantum mechanical programs allows one to obtain accurate properties using this method.

The simplest type of the $a b$ initio electronic structure calculation is the Hartree-Fock (HF) scheme, in which the instantaneous columbic electron-electron repulsion is not specifically taken in to account, only its average effect is included in the calculations. The energy obtained with this inaccurate approximation is always equal or greater than the exact energy and tend to a limiting value called the Hartree-Fock limit as the basis set size increases. A basis set is a mathematical representation of the molecular orbital within a molecule. The basis set can be interpreted as restricting each electron to a particular region of space through the use of probability functions. The use of larger basis sets include more probability density functions and thus imposes fewer constraints on electrons allowing more flexibility to occupy orbitals and more accurately approximate exact molecular orbitals. However, HF is in many cases a poor approximation to the Hamiltonian and more accurate and computationally more intensive calculations are required. Post-Hartree-Fock methods are the set of methods developed to improve on the Hartree-Fock (HF), or self-consistent field (SCF) method. They 
add electron correlation which is a more accurate way of including the repulsions between electrons than in the Hartree-Fock method where repulsions are only averaged.

Møller-Plesset perturbation theory (MP) is one of several quantum chemistry postHartree-Fock $a b$ initio methods in the field of computational chemistry. Electron correlation effects by means of Rayleigh-Schrödinger perturbation theory (RS-PT), usually to second (MP2), third (MP3) or fourth (MP4) order were added to improve on the HF method ${ }^{2}$. This method incorporates a perturbation in the Hartree-Fock Hamiltonian, $H_{o}$,

$$
H \Psi=\left(H_{o}+\lambda \mathcal{V}\right) \Psi=E \Psi
$$

where $\lambda$ is an arbitrary real parameter and $\mathcal{V}$ is the perturbation of the $H_{o}$ from the true $H$. For the MP2 method, the Eigen functions and Eigen values are expanded in a Taylor series through the second-order in the correlation potential. The total electronic energy is given by the Hartree-Fock energy plus second-order Møller-Plesset correction.

The basis set for computing the potential energy hypersurface was carefully selected considering accuracy and the computational cost. The interaction energy is the difference in energies between the dimer $\left(\mathrm{H}_{2} \mathrm{O}-\mathrm{CO}_{2}\right)$ and the monomers $\left(\mathrm{CO}_{2}, \mathrm{H}_{2} \mathrm{O}\right)$.

$$
\Delta E_{\text {int }}=E_{\mathrm{H}_{2} \mathrm{O}-\mathrm{CO}_{2}}-E_{\mathrm{H}_{2} \mathrm{O}}-E_{\mathrm{CO}_{2}}
$$

The method for computing the potential energy surface (PES) as a pair potential between $\mathrm{CO}_{2}$ and water for 18,000 points is discussed in the Section 3.2. The calculation of interaction energies is subject to basis set superposition error (BSSE) when a finite basis set is used ${ }^{3}$. When calculating the interaction energy, the atoms of interacting molecules approach one another and their basis functions overlap resulting in more basis functions for the complex than 
the individual molecules. The difference in energy from using different basis sets in each of individual calculation results in BSSE. The counterpoise (CP) approach calculates the BSSE by re-performing all the calculations using the mixed basis sets, through introducing "ghost" atoms. A ghost atom do not have protons or electrons in it, i.e. nucleus is zero, but have the same number of molecular orbital as the original atom, so it has the basis functions which can be used by other atom to increase the basis function.

\subsection{Methodology}

Guest-host pair potentials for $\mathrm{CO}_{2}$ can be developed by fitting atomic site-site potentials calculated using analytical potential models to the $a b$ initio potential energy surface. The optimum method and basis set must be determined for the accurate and efficient calculation of the $\mathrm{CO}_{2}$ and $\mathrm{H}_{2} \mathrm{O}$ interaction energies and which can be used for the calculation of the 18,000 point potential energy surface. The general source of error in the quantum calculations are error due to the electron correlation used and the error in using the incomplete basis set.

The effect of the electron correlation is examined by calculating the energies at a few selected points at increasing levels of electron correlation. For this, the $\mathrm{CO}_{2}-\mathrm{H}_{2} \mathrm{O}$ geometry was initially optimized to determine the optimum basis set for calculating the $\mathrm{CO}_{2}-\mathrm{H}_{2} \mathrm{O}$ interaction energies using MP2 level of theory and the 6-31G basis set. The optimized structure is Tshaped structure with minimum energy distance between the carbon of carbon dioxide and oxygen of water molecule, $r(\mathrm{C} \cdots \mathrm{O})=2.788 \AA$, which is in agreement with the experimentally determined $r(\mathrm{C} \cdots \mathrm{O})=2.836 \AA \hat{\text { by }}$ beterson and Klemperer ${ }^{4}$. The $r$ value is somewhat less and this might be because of the smaller basis set used for the geometry optimization. The binding 
energies were calculated for comparison between the HF, MP2 and MP4 levels of theory at various basis set to examine the optimum electron correlation method the results are presented in Table 3.1. The binding energy calculations were performed on the optimized structure. The basis set superposition error was corrected by using the full counter poise method. The counterpoise corrected binding energy between the $\mathrm{CO}_{2}$ and $\mathrm{H}_{2} \mathrm{O}$ molecules can be expressed as

$$
\Delta E_{B E, C P}=E_{\mathrm{H}_{2} \mathrm{O}-\mathrm{CO}_{2}}-E_{\mathrm{H}_{2} \mathrm{O}, \mathrm{CP}}-E_{\mathrm{CO}_{2}, \mathrm{CP}}
$$

where $\Delta E_{B E, C P}$ is the binding energy or interaction energy of $\mathrm{CO}_{2}$ and $\mathrm{H}_{2} \mathrm{O}$ complex calculated using the counterpoise method, $\mathrm{E}_{\mathrm{H}_{2} \mathrm{O}-\mathrm{CO}_{2}}$ is the total energy of the $\mathrm{CO}_{2}$ and $\mathrm{H}_{2} \mathrm{O}$ dimer calculated at any particular configuration, $E_{\mathrm{H}_{2} \mathrm{O}, C P}$ is the counterpoise corrected energy for water molecule with $\mathrm{CO}_{2}$ as a ghost atom, i.e. it has basis function but no electrons or protons in it, and $E_{\mathrm{CO}_{2}, C P}$ is the counterpoise corrected energy for carbon dioxide molecule with ghost atom for $\mathrm{H}_{2} \mathrm{O}$. Gaussian 03 is a computational tool which is used to calculate the binding energies of carbon dioxide-water dimer using the ab initio methods.

\subsubsection{Optimum method for PES calculation}

The electronic structure method (couple cluster method), $\operatorname{CCSD}(\mathrm{T})$, developed by Raghavachari et al. ${ }^{5}$, is the most accurate approximate method which represents the exact solution of the Schrödinger wave equation ${ }^{6}$. The $\operatorname{CCSD}(\mathrm{T})$ method calculations are very computationally intensive for the interaction energy calculations with higher basis set. A comparison between the various levels of theory, HF, MP2, MP3, MP4, CCSD, for the binding 
energy between the carbon dioxide and water molecule at the optimized minimum energy distance is shown in the Table 3.1. The binding energies shown in Table 3.1 were corrected for BSSE using one-half counterpoise method. When compared with the binding energy calculated by the most accurate CCSD/aug-cc-pVTZ method, the HF method is the least accurate with an absolute average deviation (AAD) of $31 \%$. The error reduces to the AAD of $1.12,3.31$ and 1.94\% using MP2, MP3 and MP4 respectively as the electron correlation was added. Therefore based on the comparison we conclude that the MP2 is an adequate level of theory for the calculation of the 18,000 point potential energy surface.

Table 3.1 $\mathrm{CO}_{2}-\mathrm{H}_{2} \mathrm{O}$ binding energies $(\mathrm{kcal} / \mathrm{mol})$ at various levels of theory and basis sets

\begin{tabular}{cccccc}
\hline Basis Set & HF & MP2 & MP3 & MP4 & CCSD(T) \\
\hline cc-pVDZ & -2.8328 & -2.7602 & -2.9131 & -2.6728 & -2.8146 \\
6-31++ G (2d,2p) & -1.9932 & -2.5710 & -2.6438 & -2.6891 & -2.5801 \\
cc-pVTZ & -2.2023 & -2.7131 & -2.7445 & -2.7817 & -2.6606 \\
aug-cc-pVTZ & -1.8452 & -2.6588 & -2.7782 & -2.7410 & -2.6889
\end{tabular}

In order to evaluate the optimum basis set, binding energy calculations were performed on the geometry optimized (minimum energy) $\mathrm{H}_{2} \mathrm{O}-\mathrm{CO}_{2}$ complex using MP2 level of electron correlation at various basis set. The binding energies calculated are shown in Table 3.2 and plotted in Figure 3.1. The BSSE was corrected by using the full counterpoise method. From Figure 3.1, we can see that the binding energy calculated with counterpoise and without counterpoise exhibits opposite trend as the basis set is increased from cc-pVDZ to cc-pV5Z. The binding energy computed decreases as the number of basis functions increases for the 
uncorrected energy making the binding weaker and for the BSSE corrected with full counterpoise method, the binding energy increases with the number of basis functions making the binding stronger. As the basis set size is increased from cc-pVDZ to cc-pV5Z, the deviations of binding energies calculated with counterpoise method and without counterpoise method decreases.

From Figure 3.2, one can determine the optimum basis set, with with trade offs of the calculation time and accuracy, to be the aug-cc-pVTZ basis set. The energy calculated at the ccpVTZ basis set level falls within the error of the maximum basis set energy, but the basis set superposition error at the cc-pVTZ level is much greater than at the aug-cc-pVTZ level. This is due to the inclusion of the diffuse functions in the augmented, aug-cc-pVTZ basis set. This aug-cc-pVTZ basis set therefore is used for the calculation of the 18,000 point potential energy surface.

Table 3.2 Binding energies calculated on $\mathrm{CO}_{2}-\mathrm{H}_{2} \mathrm{O}$ complex with geometry optimized at the MP2/6-31G level.

\begin{tabular}{ccccc}
\hline Basis Set & $\begin{array}{c}\text { NO. of } \\
\text { basis } \\
\text { functions }\end{array}$ & $\begin{array}{c}\text { Binding Energy } \\
\text { (kcal/mole) } \\
\text { Uncorrected }\end{array}$ & $\begin{array}{c}\text { Binding Energy } \\
\text { (kcal/mole) } \\
\text { Corrected by full } \\
\text { counterpoise } \\
\text { method }\end{array}$ & $\begin{array}{c}\text { Binding Energy } \\
\text { (kcal/mole) } \\
\text { Corrected by half } \\
\text { counterpoise } \\
\text { method }\end{array}$ \\
\hline cc-PVDZ & 66 & -3.7956 & -1.7246 & -2.7601 \\
6-31++G(2d,2p) & 118 & -2.8953 & -2.2466 & -2.571 \\
cc-PVTZ & 148 & -3.1960 & -2.2100 & -2.7030 \\
aug-CC-PVTZ & 230 & -2.8027 & -2.5149 & -2.6588 \\
cc-PVQZ & 280 & -2.9169 & -2.4575 & -2.6872 \\
aug-CC-PVQZ & 412 & -2.7678 & -2.6216 & -2.6947 \\
cc-PV5Z & 474 & -2.7355 & -2.5749 & -2.6552 \\
\hline
\end{tabular}




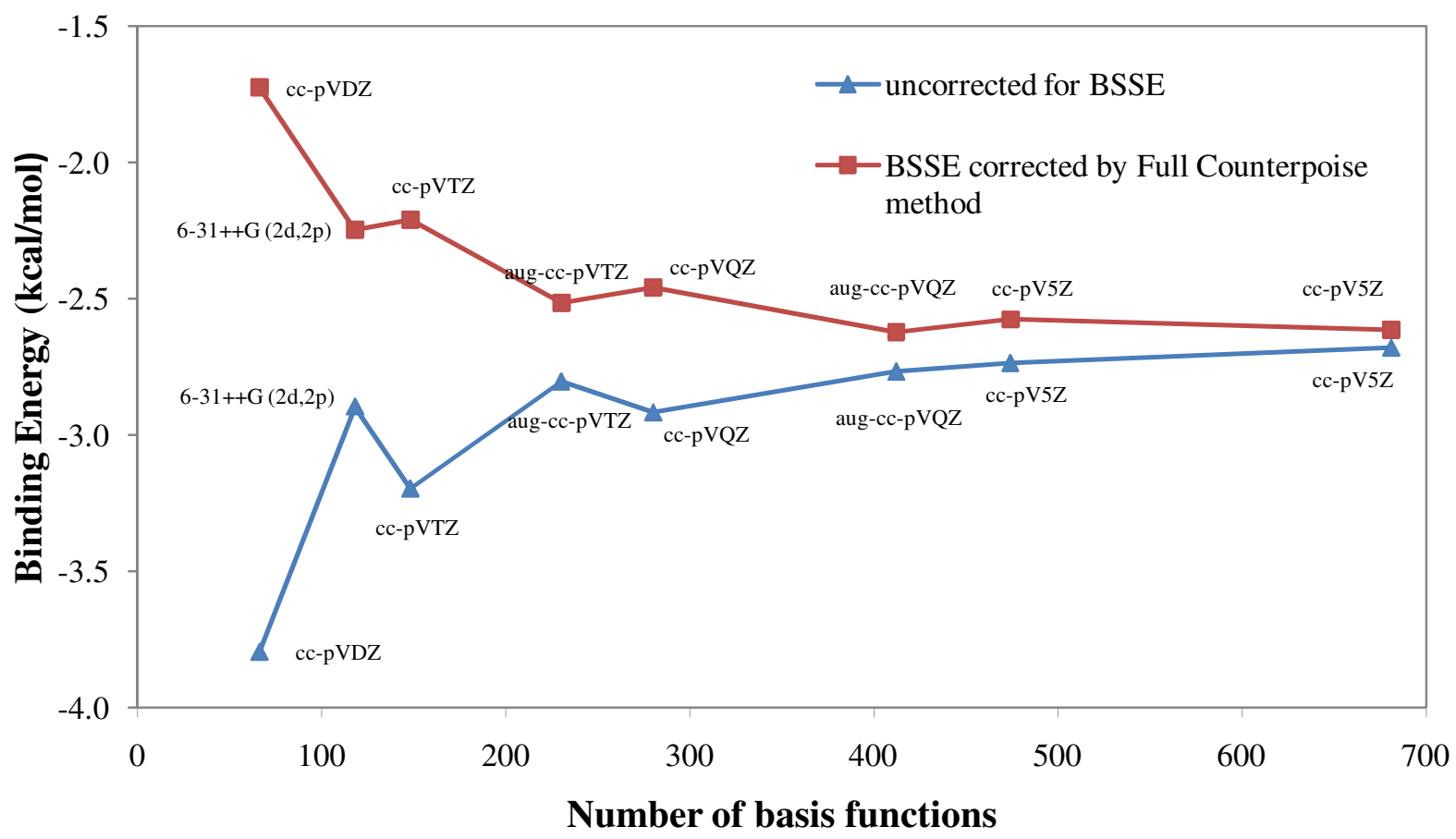

Figure 3.1 Effect of increasing basis set size on the BSSE

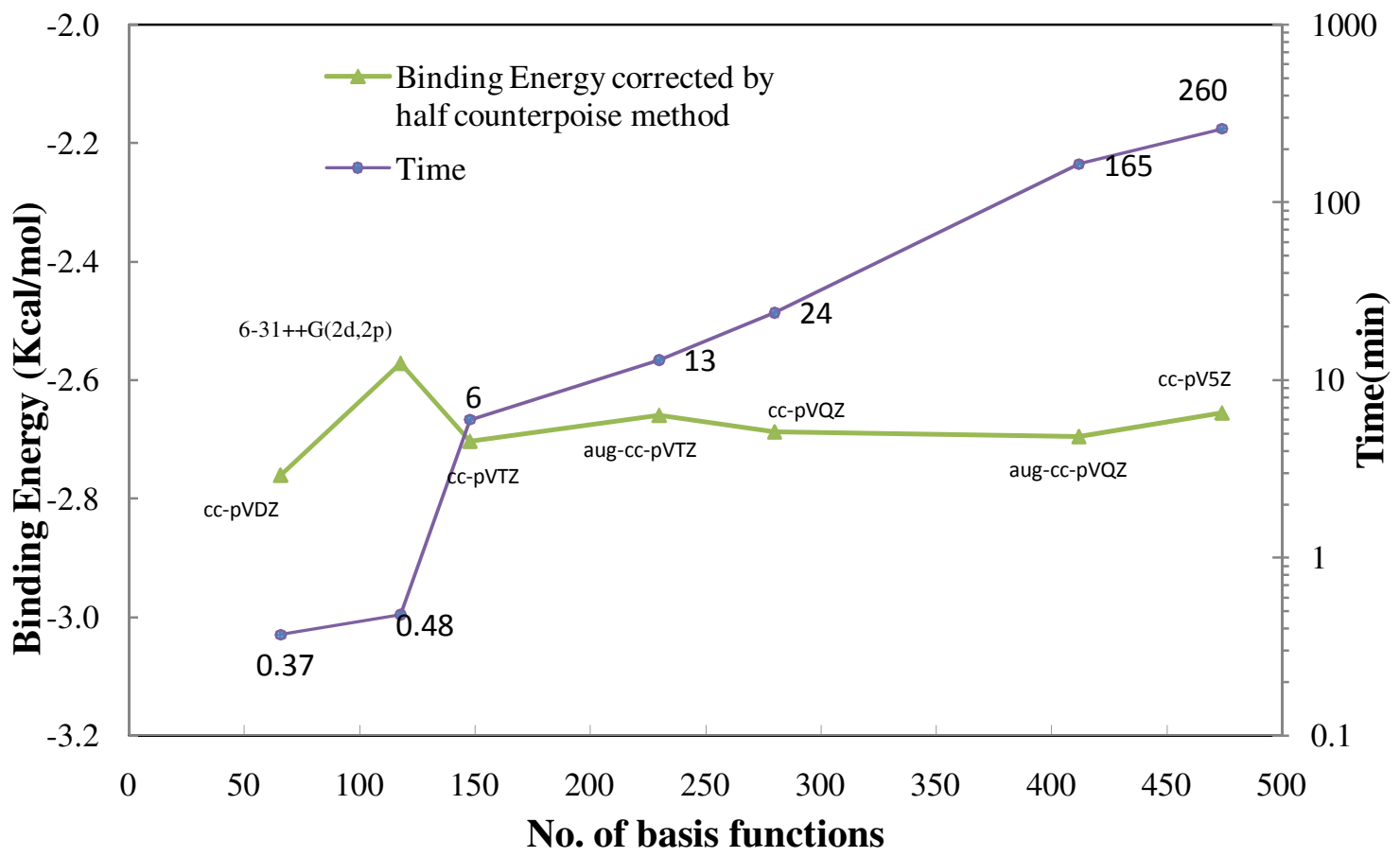

Figure 3.2 Calculation time and binding energy at each basis set for the $\mathrm{CO}_{2}-\mathrm{H}_{2} \mathrm{O}$ complex. 


\section{3 $\mathrm{Ab}$ initio intermolecular potential}

\subsubsection{Determination of potential energy surface}

The orientations between the water molecule and the carbon dioxide molecules are varied, similar to that of Cao et al. ${ }^{7}$ for $\mathrm{CH}_{4}-\mathrm{H}_{2} \mathrm{O}$, based on the actual clathrate structure. In this, the geometry of the water molecule is fixed and the geometry of the carbon dioxide molecule is varied in six dimensions for the interaction energy calculations. By inspecting the ball and stick model of the structure I clathrate hydrate, the relative orientations between guest and host water molecule fall in to two types characterizing the plane containing the water molecule as shown in Figure 3.3. The different orientations are generated by fixing the plane of water molecule in one orientation and moving the carbon dioxide guest molecule in a six-dimensional grid to different positions inside the cage. The center of mass of the carbon dioxide molecule is moved in a polar coordinate $\operatorname{system}(r, \xi, \varphi)$ with the oxygen of water molecule as the origin. This coordinate system defines the position of carbon dioxide carbon with the water oxygen as the origin. Furthermore, the rigid-body of the carbon dioxide guest molecule is rotated in its own internal coordinates, Euler angles $\alpha, \beta, \gamma$. The six-dimensional grid between the $\mathrm{CO}_{2}$ and water molecules is illustrated in Figure 3.4. The Euler angles, $\alpha$ is the degrees of angle rotated in counter clock direction along the $x$ - axis, $\beta$ is the degrees of angle rotated in counter clock direction along the $y$ - axis, $\gamma$ is the degrees of angle rotated in counter clock direction along the $z$ - axis. The limits of the $r, \xi, \varphi$ dimensions and the Euler angles are determined by the following manner.

1. The $r$ distance between the center of the carbon of $\mathrm{CO}_{2}$ molecule and the oxygen of the water molecule cannot be greater than the maximum diameter of the cage, because the 
guest molecule is entrapped in a cage. The interaction energy will become extremely repulsive when the separation distance is very small. Considering the above limitation, the separation distance, $r$, was set at 2.4-6.0 $\AA$ with 10 equally separated points were sampled in the radial direction.

2. The ranges of polar angle $\xi$, and azimuthal angle $\varphi$ were determined by moving a guest molecule some minimum distance inside a cage not too close to the cage wall. The $\xi, \varphi$ angles were selected to range from $-40^{\circ}$ to $40^{\circ}$ with five equally spaced angular points were sampled for the carbon dioxide-water space sampling.

3. The rigid-body of the carbon dioxide guest molecule is rotated in its own internal coordinates described by Euler angles $\alpha, \beta, \gamma$. The $\alpha$ is spaced between $0^{\circ}$ and $120^{\circ}$ with three points sampled at $0^{\circ}, 40^{\circ}, 80^{\circ}$. The $\beta$ is spaced between $0^{\circ}$ and $360^{\circ}$ with four points sampled at $0^{\circ}, 90^{\circ}, 180^{\circ}$ and $270^{\circ}$. The $\gamma$ is spaced between $0^{\circ}$ and $120^{\circ}$ with three points sampled at $0^{\circ}, 40^{\circ}, 80^{\circ}$.

For each radial position $r$, there are two different water planes, and $5 \times 5 \times 3 \times 4 \times 3=900$ angular orientations of the carbon dioxide molecule. Anderson et al. ${ }^{8}$ developed a site-site method, which can be used to obtain the potentials from the $900 \times 2=1,800$ interaction energies at each radial position. These potential functions can be incorporated into the configurational integral to evaluate the Langmuir constant. Prior to Anderson et al. ${ }^{8}$, Cao et al. ${ }^{7}$ performed the angle average using the Boltzmann-weighted average over the five angular orientations $(\xi, \varphi, \alpha, \beta, \gamma)$ at each radial point $r$. This angle-averaged potential results in large errors in the prediction of the cage occupancies of methane hydrate ${ }^{8}$. The work of Cao et al. was corrected by Anderson et al. by employing the site-site method instead of averaging all the five orientational and rotational degrees of freedom. This resulted in better prediction of phase 


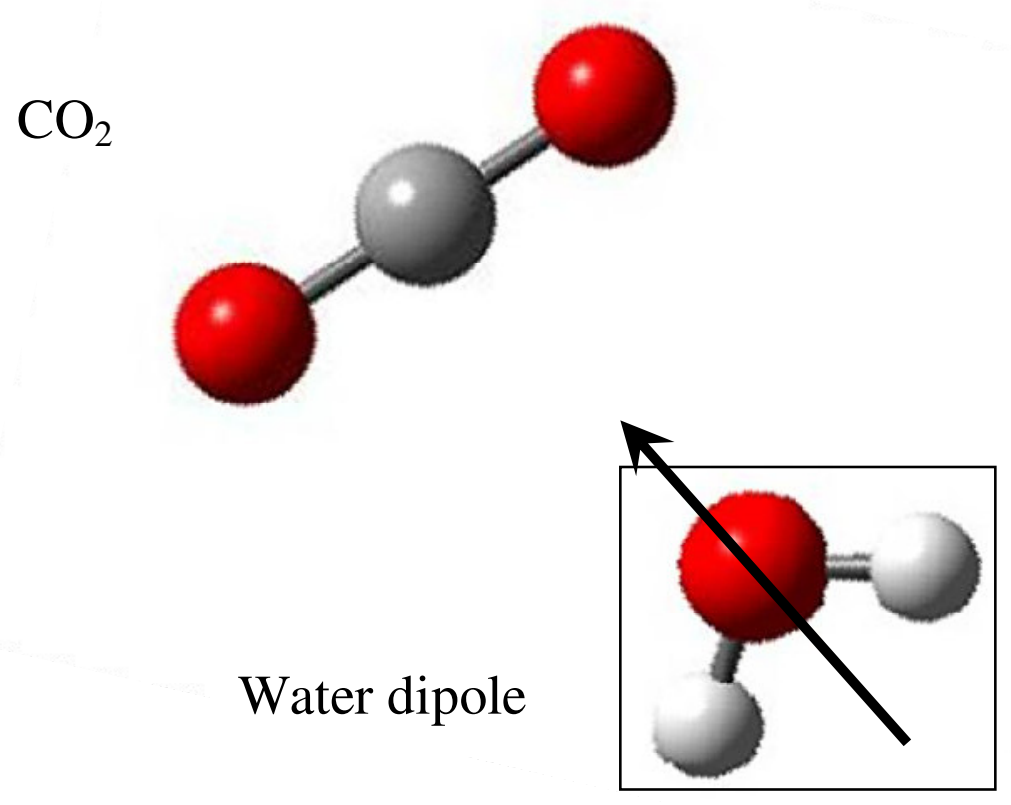

(a)

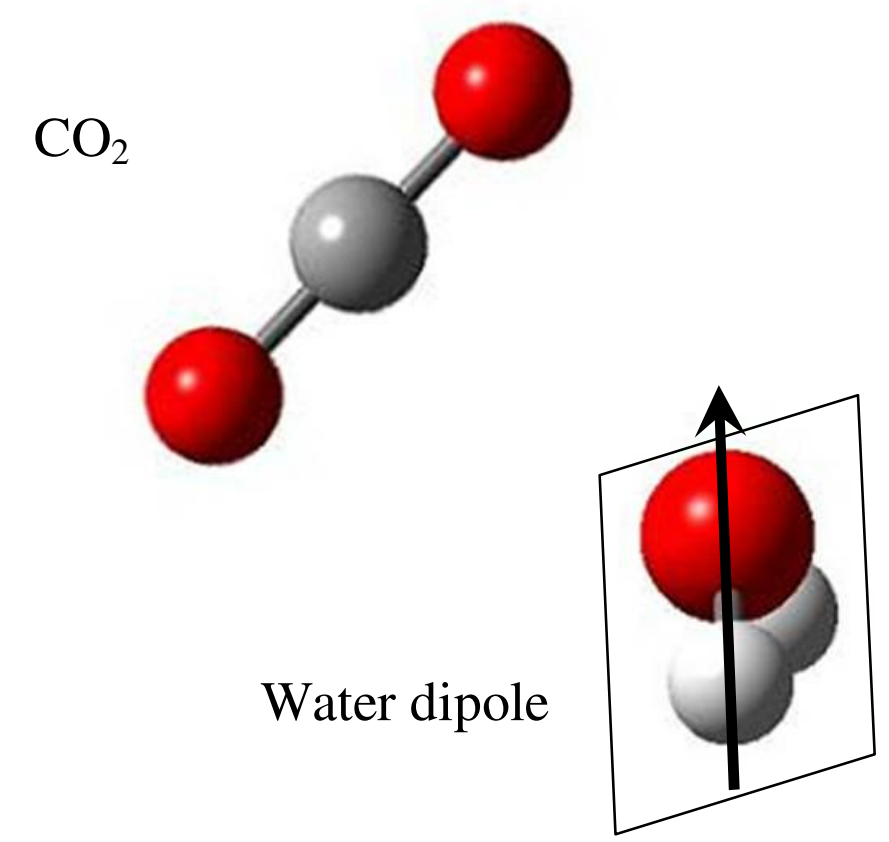

Figure 3.3 Planar Orientation of water molecule (a) water plane parallel to the page, plane-1 (b) water plane perpendicular to the page, plane- 2 
Euler angles $\alpha, \beta, \gamma$ are used to rotate carbon dioxide w.r.t. its internal $x^{\prime} y^{\prime} z^{\prime}$ coordinates

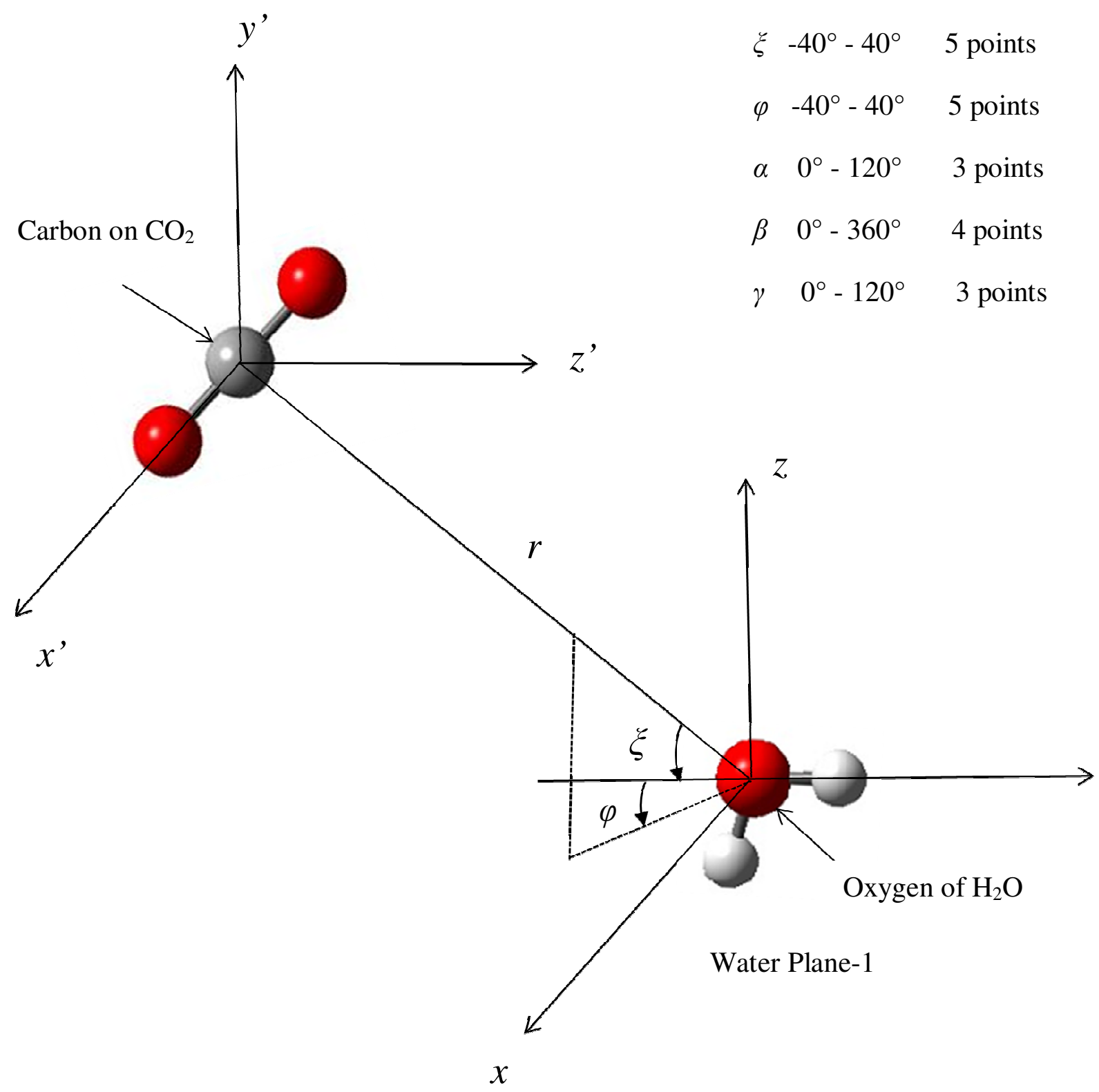

Figure 3.4 Six-dimensional orientation of carbon dioxide and water complex. 
equilibria and cage occupancies.

Therefore, a site-site model developed by Anderson et al. ${ }^{8}$ is used for the $\mathrm{CO}_{2}-\mathrm{H}_{2} \mathrm{O}$ interactions to account all six degrees of freedom. The radial position, $r$, is equally spaced in 10 different points comprising of $10 \times 1,800=18,000$ configurations between the $\mathrm{CO}_{2}-\mathrm{H}_{2} \mathrm{O}$. It was found that there was no change in binding energies calculated for the Euler angle $\alpha$ orientations. This is because of the symmetric of carbon dioxide molecule in the $x$ ' direction, see Figure 3.4. Therefore, the 18,000 configurations between carbon dioxide-water molecules are reduced to 6,000 configurations.

Table 3.3 The binding energies (kcal/mol) at aug-cc-pV5Z and aug-cc-pVTZ basis level

\begin{tabular}{|c|c|c|c|}
\hline aug-cc-pV5Z & \multicolumn{3}{|c|}{ aug-cc-pVTZ } \\
\hline $\begin{array}{c}\text { Half counterpoise } \\
\text { method }\end{array}$ & Uncorrected & Full counterpoise & Corrected energy \\
\hline 0.0033 & -0.1079 & 0.2670 & 0.0273 \\
\hline 16.0762 & 15.9112 & 16.6936 & 16.1934 \\
\hline 0.5873 & 0.4516 & 0.8272 & 0.5870 \\
\hline 0.5717 & -0.3790 & -0.0595 & -0.2638 \\
\hline-3.1150 & -2.9556 & -2.6688 & -2.7722 \\
\hline 1.3833 & 1.2493 & 1.5620 & 1.3621 \\
\hline 2.0593 & 1.9276 & 2.2401 & 2.0403 \\
\hline-0.6980 & -0.7563 & -0.6477 & -0.7171 \\
\hline-1.5898 & -1.7661 & -1.4954 & -1.6685 \\
\hline 0.0825 & 0.0326 & 0.1155 & 0.0625 \\
\hline
\end{tabular}


For the amount of counterpoise correction to be applied to the BSSE, the binding energies were computed at higher basis set, aug-cc-pV5Z basis level, for 10 different configurations and the energies were corrected with half counterpoise method. The binding energies were also computed on same 10 configurations at aug-cc-pVTZ basis level and their corrected energies at full counterpoise method were also calculated. The binding energies are given in Table 3.3. The energies are corrected for aug-cc-pVTZ basis level such that the AAD error is minimized between the energies calculated by half CP method at aug-cc-pV5Z and the combination of uncorrected and full counterpoise energies. It is found that the amount of correction to be applied for BSSE is 0.361 of corrected binding energy by full counterpoise and 0.639 of uncorrected binding energy and is given in Equation 3.5. Figure 3.5 shows the parity plot of corrected binding energies using Equation 3.5 and half counterpoise binding energies at aug-cc-pV5Z basis set level calculated at different orientations between $\mathrm{CO}_{2}$ and $\mathrm{H}_{2} \mathrm{O}$. This correction is applied to all 6,000 points energy calculations.

$$
\Delta E_{C P, \text { corrected }}=0.361 \times \Delta E_{\text {Full } C P}+0.639 \times \Delta E_{\text {uncorrected }}
$$

The input geometry file was created in Z-matrix form and Gaussian 03 was used to calculate the interaction energy at each configuration. 


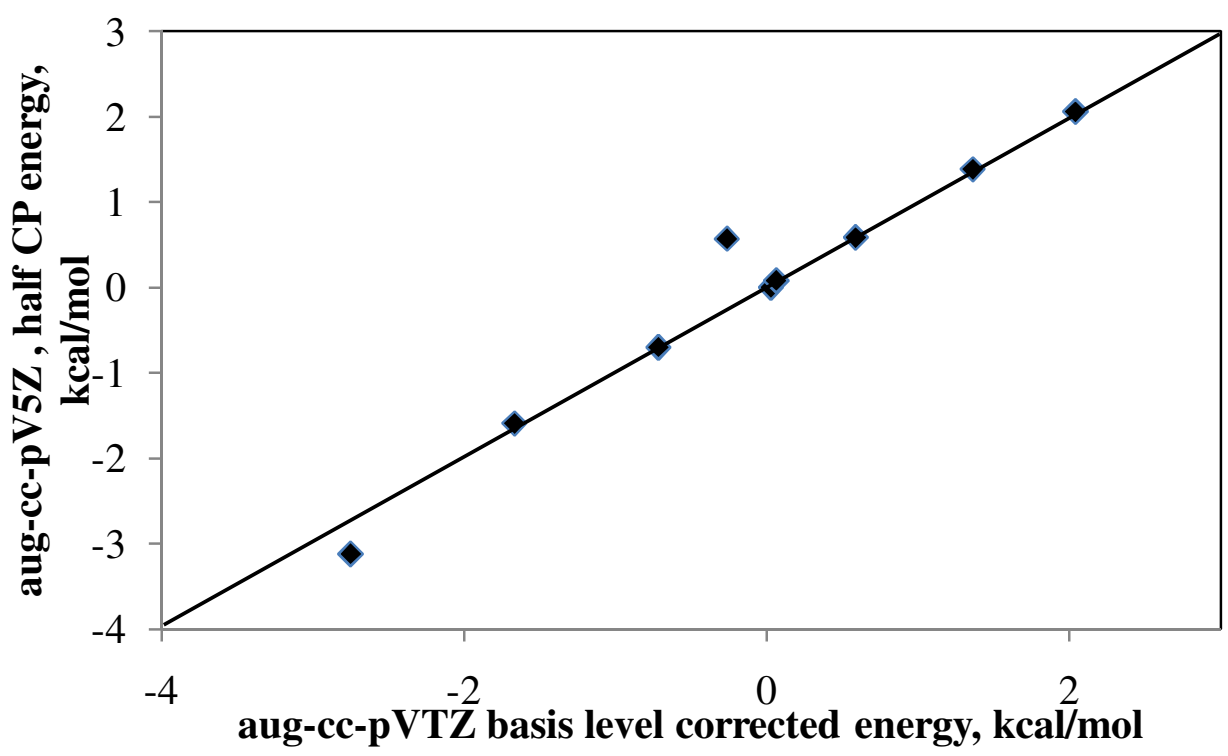

Figure 3.5 Parity plot of corrected energies of $\mathrm{CO}_{2}-\mathrm{H}_{2} \mathrm{O}$ calculated at aug-cc-pVTZ basis level w.r.t energies calculated at half counterpoise aug-cc-pV5Z basis level

\subsubsection{Potential fit to intermolecular energies}

The interaction energies calculated at 18,000 different $\mathrm{CO}_{2}-\mathrm{H}_{2} \mathrm{O}$ configurations were fit to site-site potentials based on the interactions between the center of the guest (carbon of $\mathbf{C O}_{2}$ ) molecule and the oxygen on the water $\left(\mathrm{O}_{2} \mathbf{C}-\mathbf{O H}_{2}\right)$, the oxygen of carbon dioxide with oxygen on the water $\left(\mathrm{OCO}-\mathrm{OH}_{2}\right)$, and the carbon on the $\mathrm{CO}_{2}$ with the hydrogen of the water $\left(\mathrm{O}_{2} \mathbf{C}-\right.$ $\mathbf{H O H}$ ). The interacting sites are indicated by bold symbols. The $\mathrm{O}_{2} \mathbf{C}-\mathrm{OH}_{2}$ potential captures the guest position effects, $\mathrm{O}_{2} \mathbf{C}-\mathbf{H O H}$ potential captures the $\xi$ and $\varphi$ orientations in addition to the radial dimension, $r$, and the $\mathrm{OCO}-\mathrm{OH}_{2}$ potential captures the Euler orientation of the carbon dioxide molecule with respect to the water molecule. In order to implement the $a b$ initio interaction potentials in the configurational integral for the thermodynamics calculations, the calculated potential must be accurately represented in a mathematical form. An intermolecular 
potential model is used to represent the entire guest-host interaction potential accurately. The Lennard-Jones 6-12 $2^{24}$ and exponential- $6^{24}$ potential models along with the Columbic chargecharge formula were used to fit the $a b$ initio potential. The potential forms are as follows:

Lennard-Jones 6-12:

$$
\Phi_{L J}\left(r_{i j}\right)=4 \varepsilon_{i j}\left[\left(\frac{\sigma}{r_{i j}}\right)^{12}-\left(\frac{\sigma}{r_{i j}}\right)^{6}\right]
$$

Exponential-6:

$$
\Phi_{\operatorname{exp-6}}\left(r_{i j}\right)=\frac{\varepsilon_{i j}}{1-6 / \gamma}\left[\frac{6}{\gamma} \exp \left[\gamma\left(1-\frac{r_{i j}}{r_{m}}\right)\right]-\left(\frac{r_{m}}{r_{i j}}\right)^{6}\right]
$$

The Coulombic charge-charge formula ${ }^{24}$ is given as

$$
\Phi_{\text {charge }}\left(r_{i j}\right)=\frac{1}{4 \pi \epsilon_{0}} \frac{q_{i} q_{j}}{r_{i j}}
$$

where $\frac{1}{4 \pi \epsilon_{0}}$ is the proportionality constant with $\epsilon_{0}$ as the electric constant. The proportionality constant value is $8.98755178 \times 10^{9} \mathrm{~N}-\mathrm{m}^{2} / \mathrm{C}^{2} . q_{i}, q_{j}$ are the charges on the $i^{\text {th }}$ and $j^{\text {th }}$ atom.

A nonlinear least-square fitting method was used to fit the ab initio potential data to the potential models. The $\mathrm{O}_{2} \mathbf{C}-\mathrm{OH}_{2}$ interaction was modeled using the exponential-6 potential form ${ }^{24}$, the $\mathrm{OCO}-\mathrm{OH}_{2}$ interaction was modeled using the Lennard-Jones 6-12 potential form ${ }^{24}$ and the OCO-M, $\mathrm{O}_{2} \mathbf{C}-\mathbf{H O H}, \mathrm{O}_{2} \mathbf{C}-\mathbf{M}$ and $\mathrm{OCO}-\mathrm{HOH}$ interactions were modeled using the Coulombic charge-charge form. The geometry assumed by the TIP4P model ${ }^{25}$ for $\mathrm{H}_{2} \mathrm{O}$ was 
used in the fitting of the potential, thus the additional interacting site "M" was located on the bisector of the H-O-H angle $0.15 \AA$ away from the oxygen atom toward hydrogen atom. The TIP4P water model is given in Figure 3.6, where $q_{1}=0.52$ is the charge on the hydrogen atoms and the charge on $\mathrm{M}$ is -1.04 .

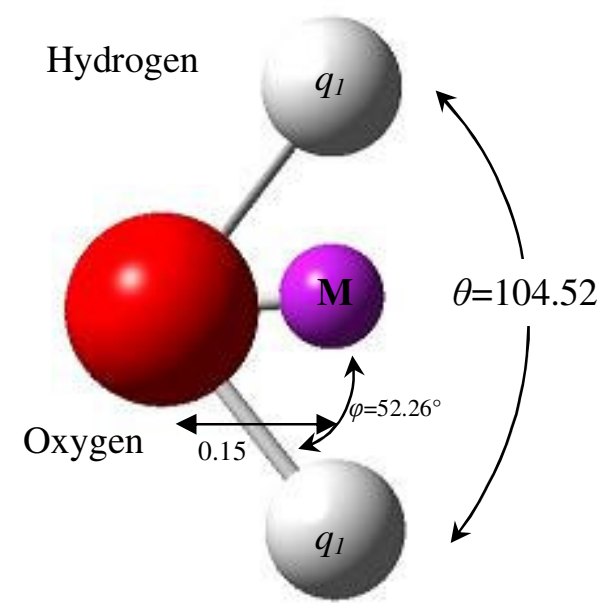

Figure 3.6 TIP4P water model

The 18,000 ab initio energies were fit to site-site potentials minimizing the Boltzmann factor-weighted objective function $\chi$ given in Equation 3.9, where by optimizing the parameters of L-J 6-12 and exponential-6. The parameter $k$ is the Boltzmann constant and temperature $T$ is $273.15 \mathrm{~K}$. The temperature, $T$, is taken as $273.15 \mathrm{~K}$ because, in nature, hydrates generally occur in and around the temperature, $T$, of $273.15 \mathrm{~K}$ and also there is no effect of variation of temperature in the minimization of objective function. The charges were held constant and are given in the Table 3.4 .

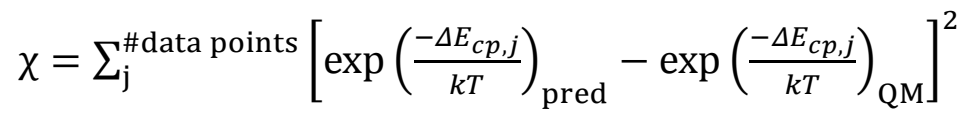


The results of the prediction of the site-site binding energies and the ab initio binding energies are shown by diagonal plot in Figure 3.7 for water plane 1 and in Figure 3.8 for water plane 2. The diagonal plot is a parity plot matrix showing the number of points of binding energies that are in the specified range for energies calculated by $a b$ initio method and by sitesite potential method. The X-axis on the diagonal plot is the site-site binding energies and the $\mathrm{Y}$-axis is the $a b$ initio binding energies. The binding energies ranging from $-2 \mathrm{kcal} / \mathrm{mol}$ to 1.25 $\mathrm{kcal} / \mathrm{mol}$ have been considered in the diagonal plot, because the attractive region is important as the energies are Boltzmann weighted when optimizing the potential parameters, see Equation 3.9. The diagonal in the Figure 3.7 and in Figure 3.8, highlighted with yellow color, represents the number of points that have the same range of binding energies calculated by $a b$ initio method and by site-site potentials.

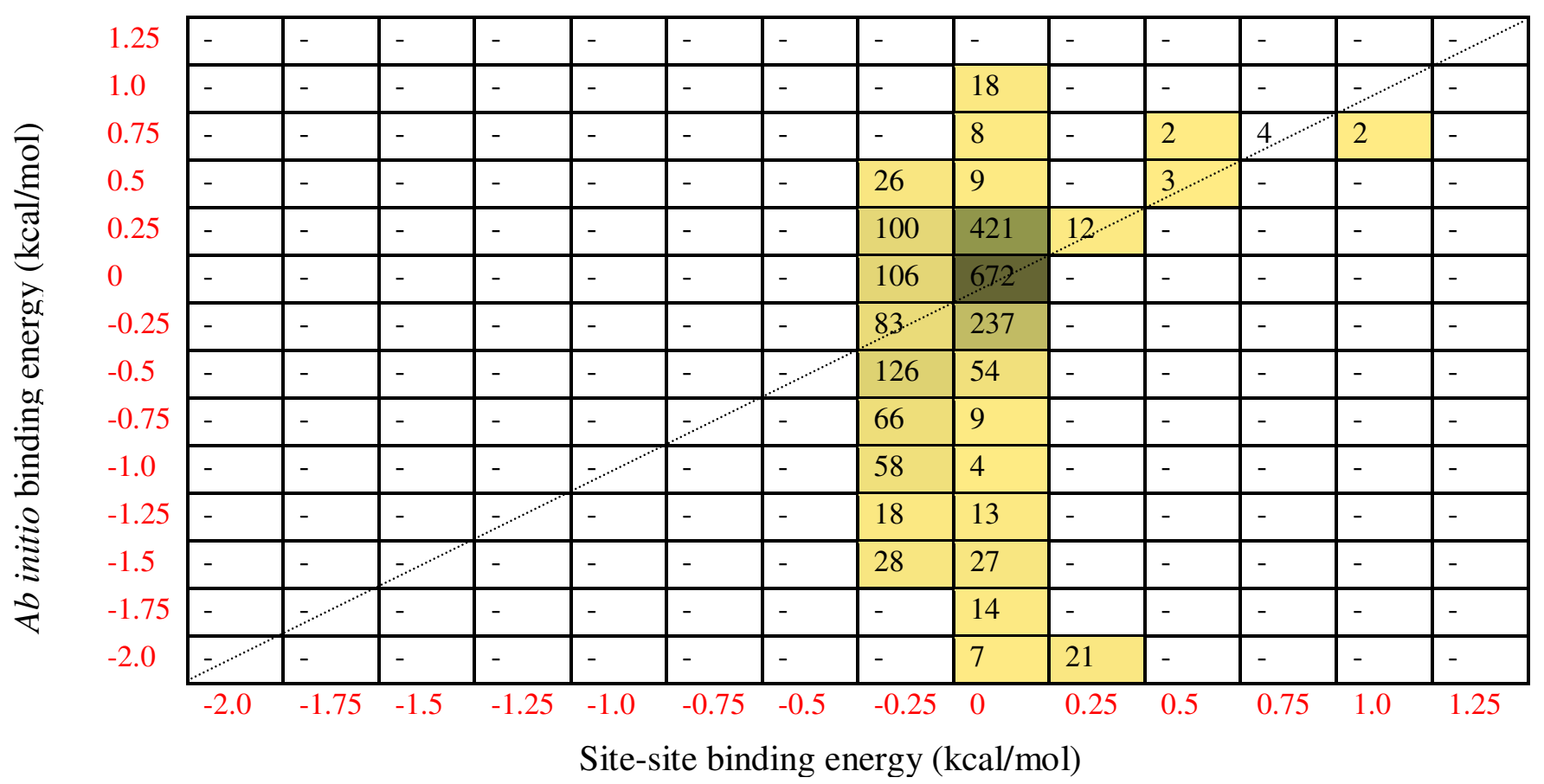

Figure 3.7 Parity plot for water plane-1 showing the number of binding energy points. 


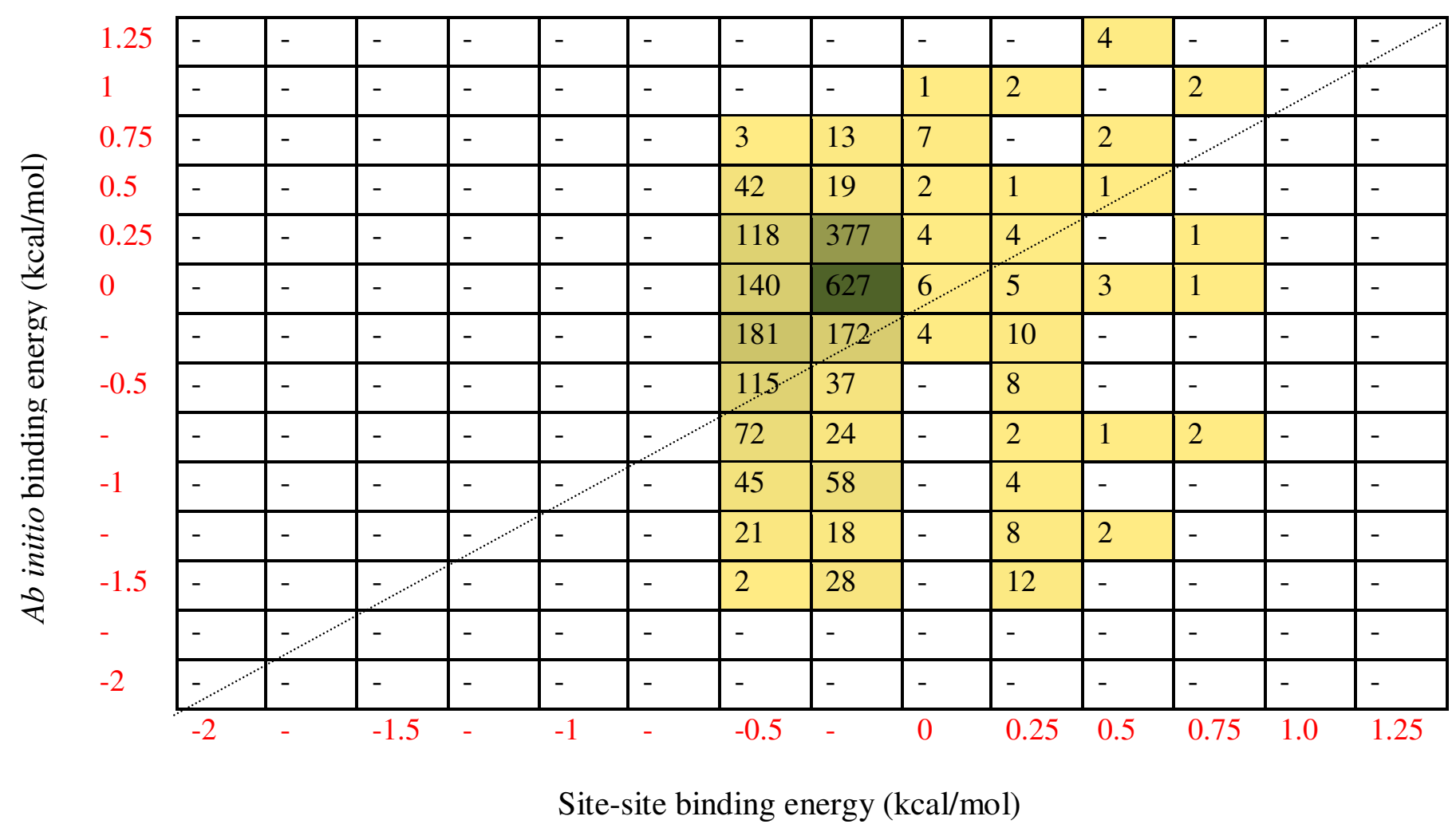

Figure 3.8 Parity plot for water plane-2 showing the number of binding energy points.

\subsubsection{Many body effects}

Klauda and Sandler ${ }^{9}$ showed that many-body effects can significantly change the total interaction energy between the guest molecule and the clathrate cage. Due to the computational limitation in time, only 15 water molecules in the pentagonal dodecahedron of structure I hydrate was considered for the interaction energy calculation. Klauda and Sandler ${ }^{9}$ showed for the methane hydrate that the two half cell calculations closely resemble the calculations of a full cage. Anderson et al. ${ }^{8}$ also calculated the many body effects for the argon guest and 
structure II pentagonal dodecahedron system and also for methane-water system. They calculated the quarter cell energies for the many-body effects. They corrected the intermolecular potentials calculated from the ab initio potential energy surface for many-body effects for argon-water system and no many-body effect was found for methane-water system.

To evaluate the many-body effects in the carbon dioxide hydrate system, initially, the half pentagonal dodecahedron of structure I with more than half water molecules, 15 water molecules, with a single guest carbon dioxide molecule is optimized for the minimum energy at MP2/6-31G level. The 15 water molecules and guest carbon dioxide system is shown in Figure 3.9. The guest molecule inside the half cage is moved in different configurations and interaction energy was calculated for this 15 water molecule and single guest $\mathrm{CO}_{2}$ molecule. Six different configurations have been obtained by moving the guest $\mathrm{CO}_{2}$ molecule towards the cage and also by rotating the $\mathrm{CO}_{2}$ molecule w.r.t 15 water molecule cell. Preliminary calculations were carried out at MP2/aug-cc-pVTZ basis level, similar to the basis set used for PES calculations, but the computational time required for the interaction energy calculation for the 16 molecule system is more than a month with the available resources. Due to the computational limitations, the interaction energies were calculated at MP2/6-31++G (2d, 2p) level for different configurations of guest in the 15 water molecule cell. The computational time required at MP2/6-31++G (2d, 2p) level basis set is around 12 hours.

The site-site model was used to calculate the total interaction energy of the many-body system. The water-water interactions within the hydrate lattice are primarily along the cage vertices and the resulting delocalization of electrons along the hydrogen bond will serve to affect the strength of the guest-hydrogen interactions ${ }^{8}$. The atomic site-site potentials obtained by optimizing the 18,000 point $a b$ initio potential energy surface were corrected for many-body 
effects. The potential parameters were optimized such that the errors of the prediction of the site-site model w.r.t the ab initio half cell calculations were minimized using the Boltzmann factor-weighted objective function $\chi$ given in Equation 3.9. The optimized site-site potential parameters are listed in Table 3.4. Figure 3.10 shows the results of the binding energies calculated on the 15 water molecules- $\mathrm{CO}_{2}$ system.

Table 3.4 $\mathrm{CO}_{2}-\mathrm{H}_{2} \mathrm{O}$ potential parameters by site-site model

\begin{tabular}{|c|c|c|c|c|c|c|}
\hline & \multicolumn{3}{|c|}{ Exp -6 } & \multicolumn{2}{|c|}{ L-J 6-12 } & \multirow[t]{2}{*}{ Charge } \\
\hline & $\varepsilon / k(\mathrm{~K})$ & $r_{m}(\AA)$ & $\gamma$ & $\varepsilon / k(\mathrm{~K})$ & $\sigma(\AA)$ & \\
\hline $\mathrm{O}_{2} \mathbf{C}-\mathbf{O H}_{2}$ & 89.63 & 3.8050 & 10.6958 & & & \\
\hline $\mathrm{OCO}-\mathbf{O H}_{2}$ & & & & 77.4 & 3.060 & \\
\hline $\mathrm{CO}_{2}$ & & & & & & 0.652 \\
\hline $\mathrm{CO}_{2}$ & & & & & & -0.326 \\
\hline $\mathrm{H}_{2} \mathrm{O}$ & & & & & & 0.0 \\
\hline $\mathrm{H}_{2} \mathrm{O}$ & & & & & & 0.52 \\
\hline M & & & & & & -1.04 \\
\hline
\end{tabular}




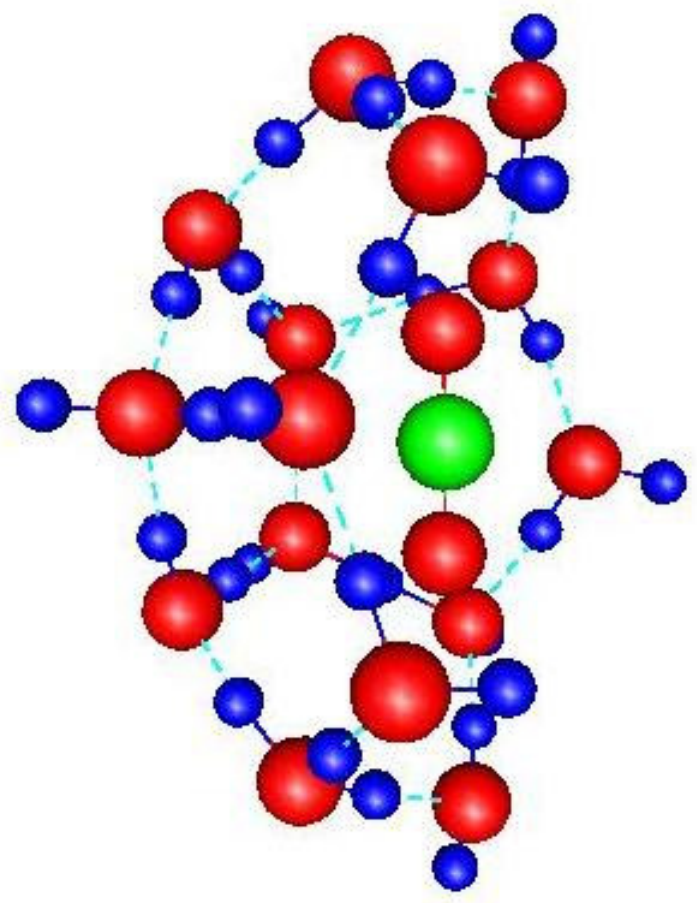

Figure 3.9 Single guest $\mathrm{CO}_{2}$ and 15 water molecules of the pentagonal dodecahedron of the structure I hydrate

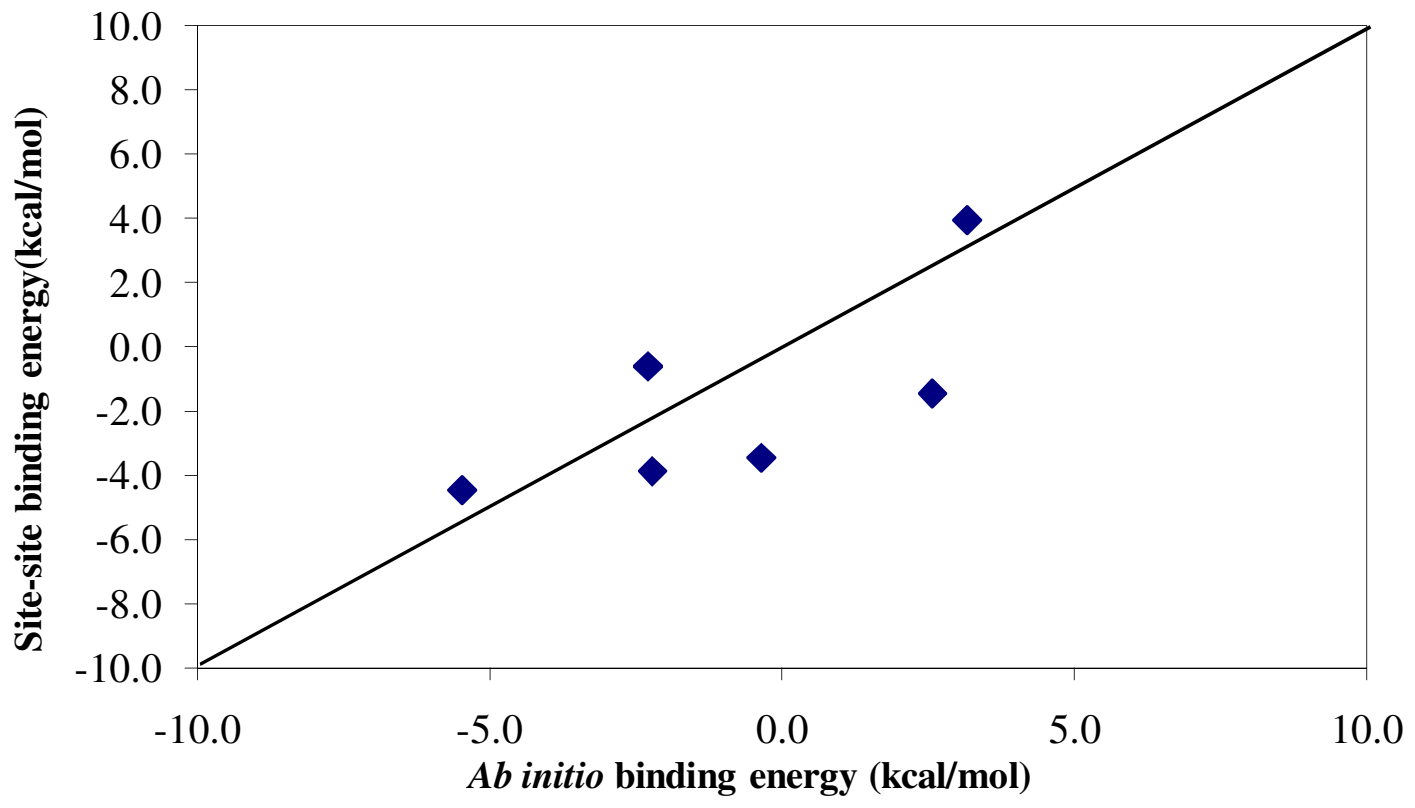

Figure 3.10 Parity plot of corrected site-site predicted 15 water molecule-carbon dioxide interaction energies. 


\subsection{Reference parameters}

Holder et al. ${ }^{10}$ first developed an empirical correlation method to calculate the reference chemical potential difference, $\Delta \mu_{w}{ }^{0}$, and enthalpy difference, $\Delta H_{w}{ }^{0}$. They calculated the reference parameters for structure I hydrate using the cyclopropane data of Dharmawardhana et al. ${ }^{11}$ The reference properties are critical inputs to the statistical model to accurately calculate the cage occupancy and phase equilibrium of the hydrate. Many investigators typically determine two critical thermodynamic reference parameters, $\Delta \mu_{w}{ }^{0}$ and $\Delta H_{w}{ }^{0}$. Several methods, both experimental and analytical, have been adopted in the past to determine the reference parameters. The reference parameters, $\Delta \mu_{w}{ }^{0}$ and $\Delta H_{w}{ }^{0}$, given by earlier researchers for structure I are given in Table 2.1. Holder et al. ${ }^{12}$ suggested that the reference chemical potential difference, $\Delta \mu_{w}{ }^{0}$, varies with the size of the guest molecule instead of using a single value for all the guest molecules, as there is a distortion in the lattice with the size of the guest molecule is increased. Pradhan ${ }^{13}$ found that the reference chemical potential difference value increases with the increase in size of the guest molecule by fitting the experimental data while slightly adjusting the Kihara parameters for some guest molecules. Carbon dioxide being the large molecule compared to the small molecule like methane might cause the lattice distortion. The molecular diameter of $\mathrm{CO}_{2}$ molecule is $5.12 \AA$ and for the $\mathrm{CH}_{4}$ is $4.36 \AA$. The reference parameters for structure I carbon dioxide gas hydrate is calculated using the method developed by Holder et al. ${ }^{10}$ and the ab initio pair potential for $\mathrm{CO}_{2}-\mathrm{H}_{2} \mathrm{O}$ interactions.

Holder et al. ${ }^{10}$ integrated and rearranged the Equations 2.8, 2.9 and 2.10 in the following rigorous form 


$$
\begin{aligned}
\frac{\Delta \mu_{w}{ }^{\beta-H}(T, P)}{R T} & +\frac{\Delta C_{P}{ }^{\beta-L, \alpha}\left(T_{0}\right)-b T_{0} \ln \left(T / T_{0}\right)}{R}-\frac{2 \Delta C_{P}{ }^{\beta-L, \alpha}\left(T_{0}\right)-b^{\beta-L, \alpha} T_{0}{ }^{2}}{2 R}\left[\frac{1}{T_{0}}-\frac{1}{T}\right]+ \\
& \frac{b^{\beta-L, \alpha}}{2 R}\left(T-T_{0}\right)-\frac{P \Delta V_{w}{ }^{\beta-L, \alpha}}{R T}+\ln a_{w}=\frac{\Delta \mu_{w}{ }^{0}}{R T}+\frac{\Delta H_{w}{ }^{\beta-L, \alpha}\left(T_{0}\right)}{R}\left[\frac{1}{T}-\frac{1}{T_{0}}\right]
\end{aligned}
$$

The reference temperature, $T_{o}$, is the ice point temperature. In case of methane hydrate the ice point temperature $T_{o}=273.15 \mathrm{~K}$ and in case of carbon dioxide hydrate, $T_{o}$ is $271.75 \mathrm{~K}$. The depression in the ice point temperature for $\mathrm{CO}_{2}$ hydrate is due to the high solubility of carbon dioxide in water. So in the case of carbon dioxide hydrate, if the temperature is greater than 271.75 K, the water is in liquid phase then

$$
\begin{aligned}
& \Delta H_{w}{ }^{\beta-L, \alpha}\left(T_{0}\right)=\Delta H_{w}{ }^{\beta-L}\left(T_{0}\right)=\Delta H_{w}{ }^{\beta-\alpha}\left(T_{0}\right)+\Delta H_{w}{ }^{L-\alpha}\left(T_{0}\right) \\
& =\Delta H_{w}{ }^{0}+\Delta H_{w}{ }^{L-\alpha}\left(T_{0}\right)
\end{aligned}
$$

and for temperatures less than $271.75 \mathrm{~K}$, the $\Delta H_{w}{ }^{\beta-L, \alpha}\left(T_{0}\right)$ is expressed as Equation 3.17.

$$
\Delta H_{w}^{\beta-L, \alpha}\left(T_{0}\right)=\Delta H_{w}^{0}
$$

where $\Delta H_{w}{ }^{L-\alpha}\left(T_{0}\right)$ is the latent heat of ice. The values of the constants are given in Table 3.4. If the left hand side of the Equation 3.15 is defined as $Y$ then the Equation 3.15 has the form

$$
Y=\frac{\Delta \mu_{w}^{0}}{R T}+\frac{\Delta H_{w}{ }^{0}}{R}\left[\frac{1}{T}-\frac{1}{T_{0}}\right]
$$

where $Y$ is a function of experimental conditions, temperature $T$ and pressure $P$, and other constants, namely $\Delta V_{w}{ }^{\beta-L, \alpha}, \Delta C_{P}{ }^{\beta-L, \alpha}\left(T_{0}\right)$ and $b$. If the fundamental thermodynamic equations are correct and if one assumes that the constants in Table 3.5 are, in fact, constant, a plot of $Y$ vs. $\left[1 / T-1 / T_{0}\right]$ should yield a straight line and whose intercept and slope will yield $\Delta \mu_{w}{ }^{0}$ and $\Delta H_{w}{ }^{0}$ respectively. 
Table 3.5 Heat capacity and volumetric reference properties between the empty hydrate lattice and fluid phase (liquid water or ice)

\begin{tabular}{|ccc|}
\hline Constants & Reference \\
\hline$\Delta \mathrm{V}_{w}{ }^{\beta-\alpha}\left(\mathrm{m}^{3} / \mathrm{mol}\right)$ & $3.0 \times 10^{-6}$ & 14 \\
$\Delta \mathrm{V}_{w}{ }^{L-\alpha}\left(\mathrm{m}^{3} / \mathrm{mol}\right)$ & $-1.598 \times 10^{-6}$ & 15 \\
$\Delta \mathrm{H}_{w}{ }^{L-\alpha}(\mathrm{J} / \mathrm{mol})$ & 6009.5 & \\
$\Delta \mathrm{C}_{p}{ }^{\beta-\alpha}\left(T_{0}\right)(\mathrm{J} / \mathrm{mol} . \mathrm{K})$ & 0.565 & 16 \\
$b^{\beta-\alpha}$ & 0.002 & \\
$\Delta \mathrm{C}_{p}{ }^{\beta-L}\left(T_{0}\right)(\mathrm{J} / \mathrm{mol} . \mathrm{K})$ & -37.32 & \\
$b^{\beta-L}$ & 0.179 & \\
\hline
\end{tabular}

With the intermolecular potentials developed for the carbon dioxide-water system given in Table 3.2 from the ab initio potential energy surface, Langmuir constants are calculated by integrating a six dimensional integral of Equation 3.12. In the Langmuir constant calculation, the contributions of interactions between the guest and host molecules from first water shell to fourth water shell were included. The cage occupancy probabilities are calculated at any specific temperature of interest from Langmuir constant, from Equation 3.11. The $\Delta \mu_{w}{ }^{\beta-H}(T, P)$ is calculated from the Equation 3.9. The only experimental data needed to calculate the reference parameters are the readily available carbon dioxide hydrate $P-T$ equilibrium. The plot for the reference parameters are shown in Figure 3.11. The P-T equilibrium data is obtained from Sloan and $\mathrm{Koh}^{1}$. Using a linear regression analysis, the reference thermodynamic parameters obtained are $\Delta \mu_{w}{ }^{o}=1204 \pm 3 \mathrm{~J} / \mathrm{mol}$ and $\Delta H_{w}{ }^{o}=1190 \pm$ $12 \mathrm{~J} / \mathrm{mol}$. The estimation of error in the calculation of reference parameters was found by 
calculating the $95 \%$ confidence intervals on the regression. The experimental error in $P-T$ equilibrium data measurement will introduce some uncertainty, but experimental errors were not included in the reference parameters calculation.

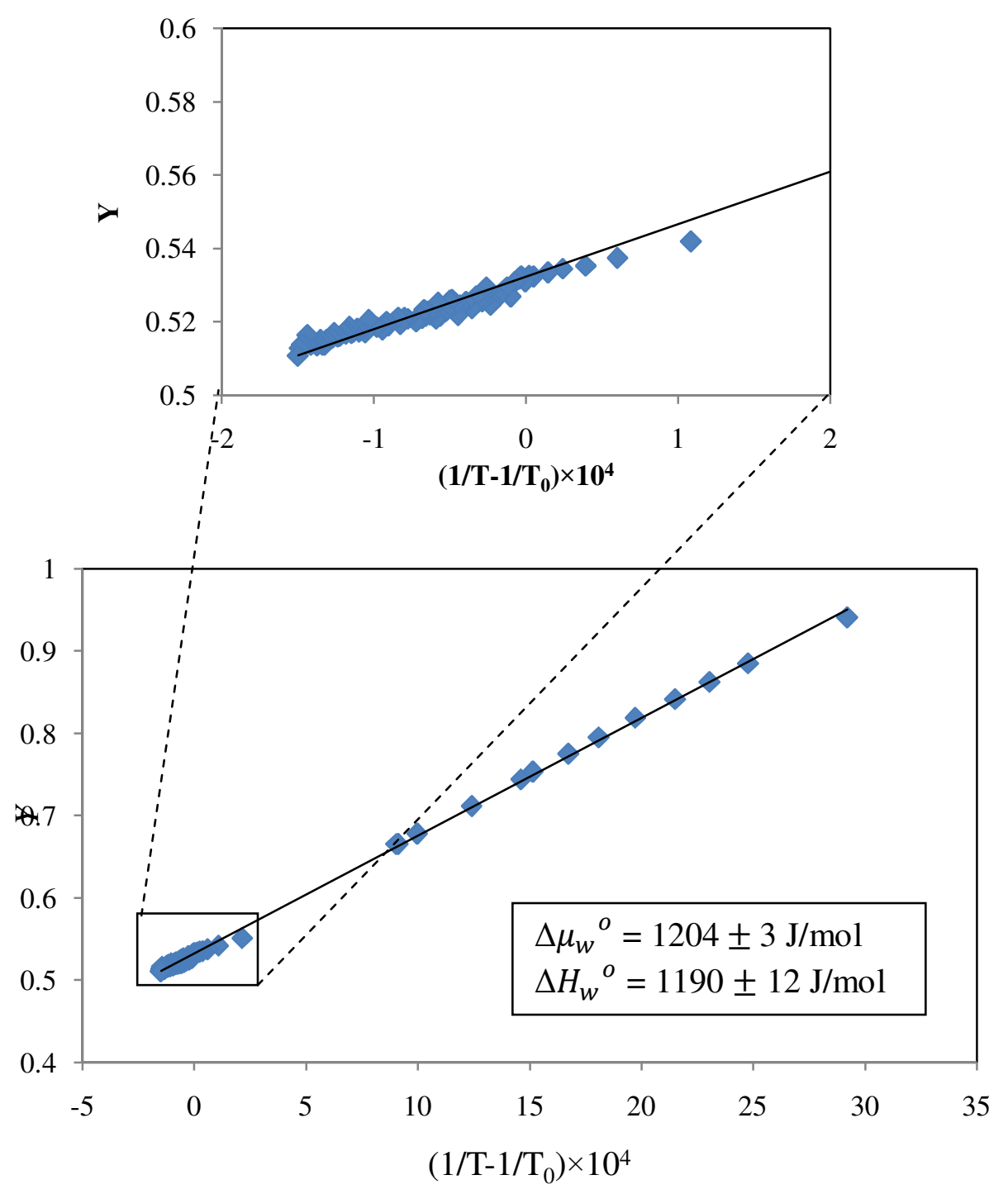

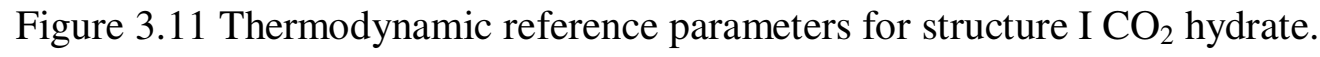

The source of experimental data: Miller and Smythe ${ }^{27}$, Flabella $^{28}$, Larson $^{29}$, Robinson and Mehta $^{30}$, Deaton and Frost ${ }^{31}, \mathrm{Ng}$ and Robinson ${ }^{32}$, Unruh and Katz ${ }^{33}$, Adisasmito et al. ${ }^{34}$, Ohgaki et al. ${ }^{35}$. 
There are a number of intermolecular potential models for carbon dioxide that accurately predicts the solubility, however the most widely used intermolecular potentials for carbon dioxide is the EPM2 potential model developed by Harris and Yung ${ }^{23}$. In the EPM2 model, Lennard-Jones interactions and point charges centered on each atom are used. The potential was obtained by fitting to VLE data. The EPM2 model potentials works very well for the solubility of carbon dioxide in the solvents, but this study will show that it fails to predict the cage occupancy and phase equilibrium pressure when applied to hydrates. The intermolecular potentials for the carbon dioxide-water complex are calculated by using the Lorentz-Berthelot ${ }^{24}$ combining rules given in Equations 3.20 and 3.21. The potentials for water are from TIP4P model.

$$
\begin{aligned}
\sigma_{i j} & =\frac{\sigma_{i i}+\sigma_{j j}}{2} \\
\varepsilon_{i j} & =\sqrt{\varepsilon_{i i} \varepsilon_{i i}}
\end{aligned}
$$

Similar to the reference parameters calculated as above using the $a b$ initio intermolecular potentials, the reference parameters are calculated with the intermolecular potentials calculated using the Lorentz-Berthelot combining rules and Harris and Yung potentials for $\mathrm{CO}_{2}$ with TIP4P model for water. The reference parameters obtained by using the Harris and Yung potentials are $\Delta \mu_{w}{ }^{o}=178.4 \pm 3 \mathrm{~J} / \mathrm{mol}$ and $\Delta{H_{w}}^{\circ}=95.8 \pm 12 \mathrm{~J} / \mathrm{mol}$. The reference parameters obtained well outside the range obtained by earlier researchers either numerically or experimentally given in Table 2.1 for structure I hydrate. This shows the inability of the Harris and Yung potentials to accurately model carbon dioxide hydrates using the van der Waals and Platteeuw ${ }^{17}$ model frame work. This also would call into question its applicability for molecular dynamic simulations. 


\subsection{Prediction of Phase Equilibria}

In order to predict the three-phase hydrate equilibrium pressure at any given temperature, the algorithm discussed in Section 2.4 was used in an iterative manner to obtain the converged pressures which satisfies the van der Waals and Platteeuw ${ }^{17}$ model. Using the regressed reference parameters given in Figure 3.11 for structure I carbon dioxide hydrate and the constants in Table 3.4 for structure I hydrate, the equilibrium pressure of $\mathrm{CO}_{2}$ hydrate at a given temperature is calculated. The algorithm for calculating the equilibrium pressure at a

particular temperature by an iterative process is given in Figure 3.8. Figure 3.9 and 3.10 compares the equilibrium pressure of $\mathrm{CO}_{2}$ hydrate at various temperatures ranging from $155 \mathrm{~K}$ to $283.3 \mathrm{~K}$ with the experimental data. The absolute average deviation is less than $2 \%$ from the experimental data. 


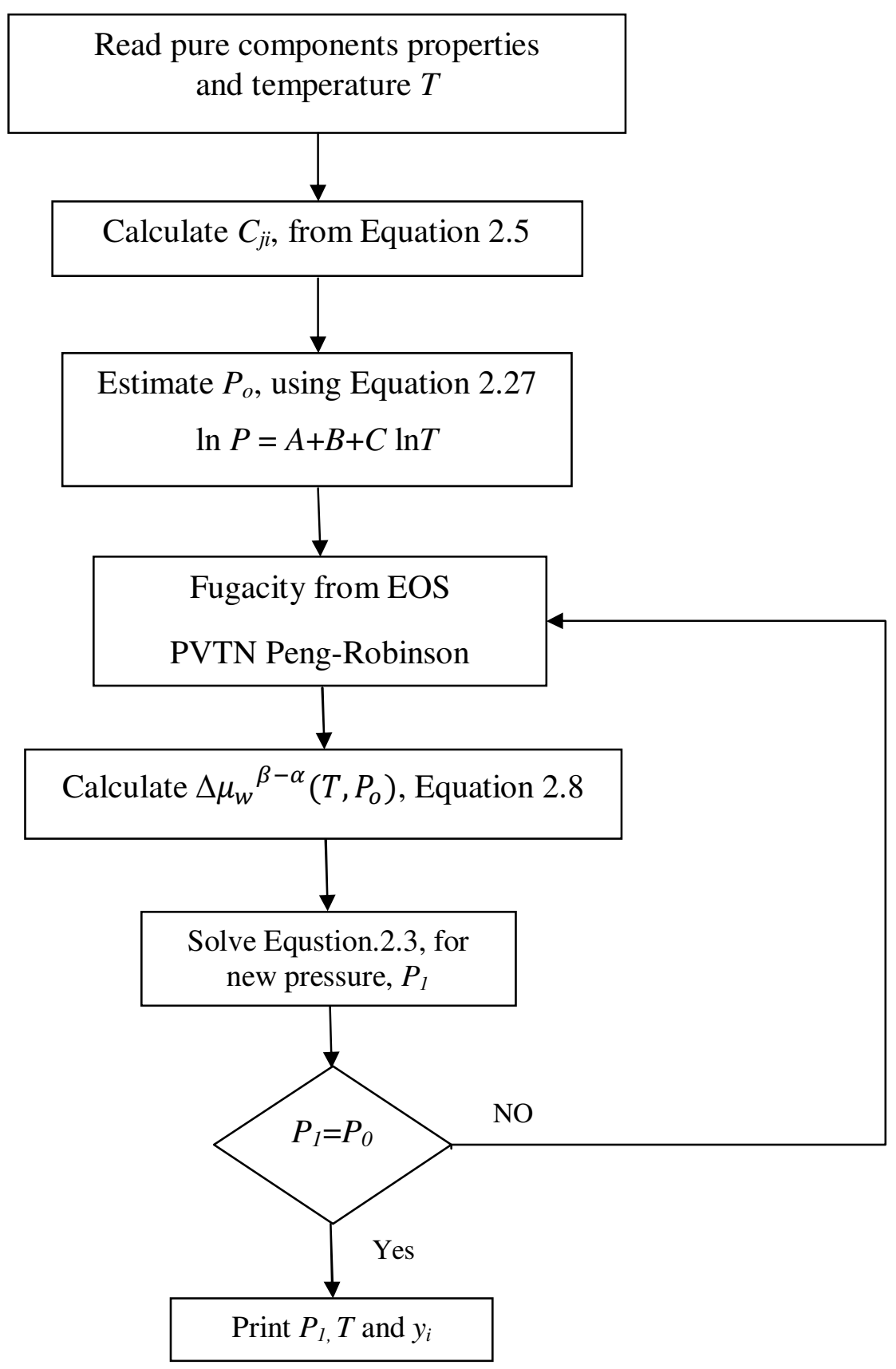

Figure 3.12 Algorithm to calculate the phase equilibrium and cage occupancy 


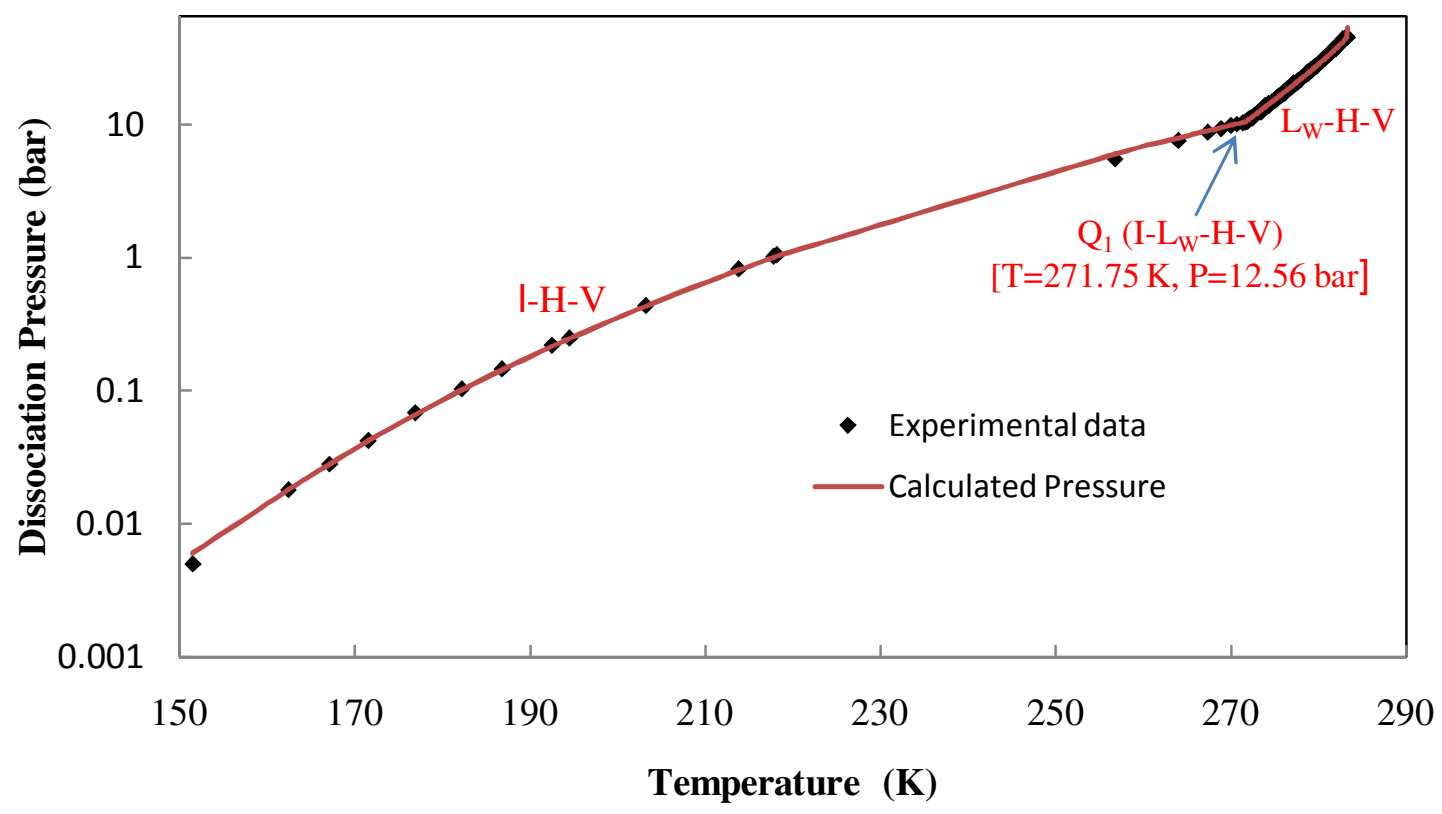

Figure 3.13 Calculation of $\mathrm{CO}_{2}$ hydrate equilibrium dissociation pressure using ab initio sitesite potentials and regressed reference parameters for $\mathrm{CO}_{2}$.

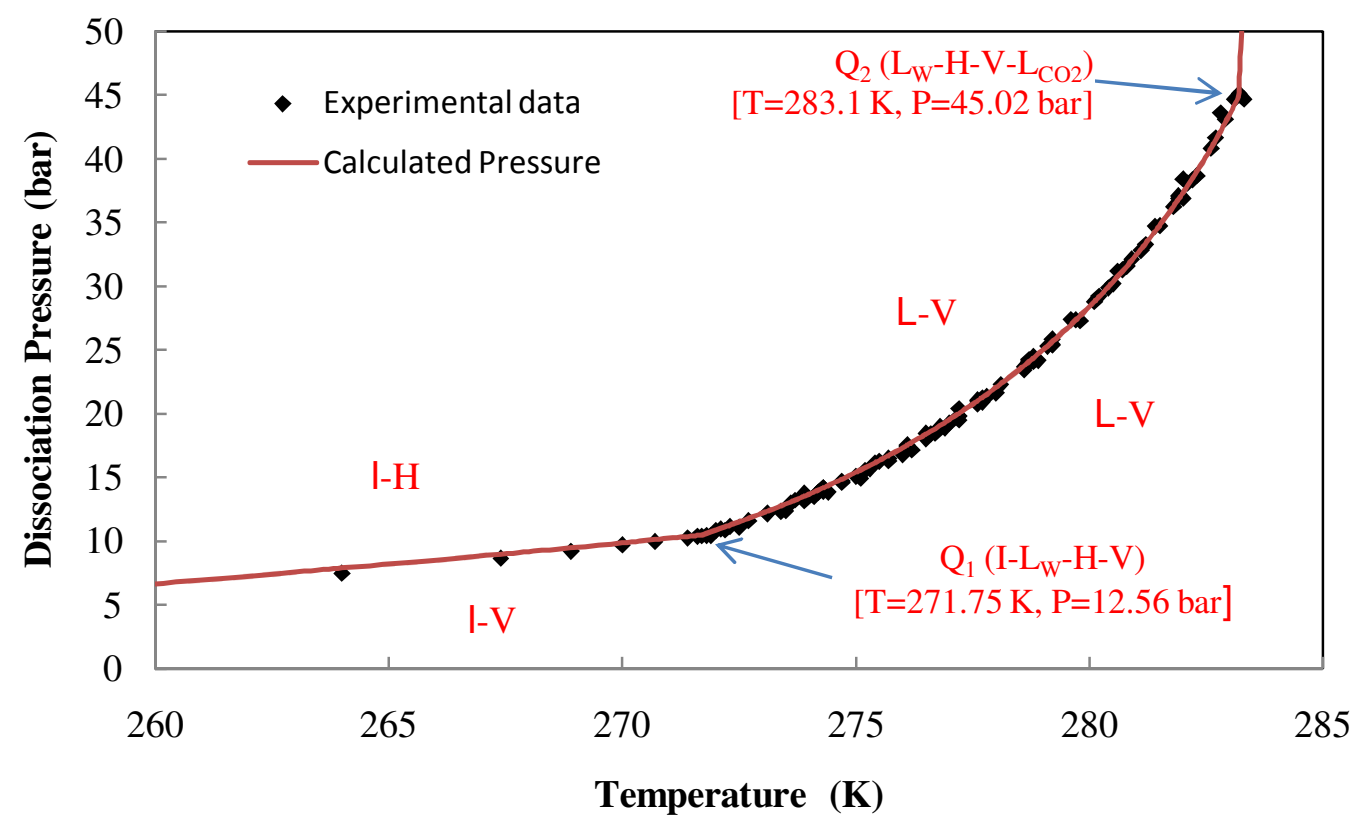

Figure 3.14 Calculation of $\mathrm{CO}_{2}$ hydrate equilibrium dissociation pressure for $T>260 \mathrm{~K}$, using $a b$ initio site-site potentials and regressed reference parameters for $\mathrm{CO}_{2}$.

The source of experimental data: Miller and Smythe ${ }^{27}$, Flabella $^{28}$, Larson $^{29}$, Robinson and Mehta $^{30}$, Deaton and Frost ${ }^{31}, \mathrm{Ng}$ and Robinson ${ }^{32}$, Unruh and Katz ${ }^{33}$, Adisasmito et al. ${ }^{34}$, Ohgaki et al. ${ }^{35}$. 


\subsection{Cage occupancies}

Cage occupancies, the fraction of each cage occupied by a guest molecule, are important as it tells the amount of gas stored in the hydrate or the amount of gas that can be stored in the hydrate. Composition of the hydrate at in-situ temperature and pressure must be known in order to fully understand the thermodynamics and kinetics of the gas hydrate formation and decomposition. The hydration number, $n$, can be determined from the cage occupancies, as the hydration number is the average number of water molecules per guest molecule in the hydrate. For structure I hydrate, the hydration number can be calculated using Equation 3.19. For fully occupied large, $\theta_{L}=1$ and small cages, $\theta_{s}=1$, of structure I gas hydrate the hydration number calculated using Equation 3.1 is 5.75.

$$
n=\frac{23}{3 \theta_{L}+\theta_{s}}
$$

Spectroscopic measurements such as NMR and Raman have been used by different researchers to calculate the cage occupancy in which the integrated signal intensity ratios of the guests in the two hydrate cavities are measured ${ }^{26}$. The signal intensity ratios between peaks for guests in each cage type reproduce the ratios of the cage occupancies $\left(\theta_{S} / \theta_{L}\right.$, small cage to large cage) of the guest in the lattice cages. The cage occupancies determined by the Henning et al. ${ }^{19}$ from neutron diffraction studies for the $\mathrm{CO}_{2}$ guest were more than $95 \%$ for the large cavities $\left(5^{12} 6^{2}\right)$, and for the small cages $\left(5^{12}\right)$ is in the range of $60 \%$ to $80 \%$. This gives the hydration numbers between 6.05 and 6.67. They prepared the sample at temperatures between $263 \mathrm{~K}$ and $278 \mathrm{~K}$ with pressures well above the equilibrium pressures, around $60 \mathrm{~atm}$. The cage

occupancies reported by Udachin et al. ${ }^{20}$ from the single crystal X-ray diffraction studies were $100 \%$ for the large cage $\left(\theta_{L}\right)$ and $71 \%$ for the small cage $\left(\theta_{S}\right)$; this yields the hydration number 
of 6.20. They prepared the crystal at temperature $276 \mathrm{~K}$ in the presence of excess liquid $\mathrm{CO}_{2}$ and pressure almost twice that of the equilibrium condition, at $38 \mathrm{~atm}$.

The cage occupancy reported for carbon dioxide hydrate using the experimental techniques is that the large cage is almost fully occupied, but there is a large discrepancy in predicting the small cage occupancy ${ }^{19-21}$. The small cage occupancies reported are in the range of $60-80 \%$. In all the experimental measurements except by Ripmeester and Ratcliff ${ }^{21}$ the $\mathrm{CO}_{2}$ hydrate samples prepared for determining the cage occupancies and hydration numbers were well above the equilibrium pressures and these higher pressures during the synthesis produce higher occupancies. Ripmeester and Ractliff ${ }^{21}$ prepared a sample under equilibrium conditions, at temperature $268 \mathrm{~K}$ and pressure of 9.9 bar, gave a lower limit to the hydration number of 7.0 for $\mathrm{CO}_{2}$ hydrate. They used solid state NMR to measure the relative cage occupancy, $\theta_{S} / \theta_{L}$, of 0.32 and assumed a $\Delta \mu_{w}{ }^{0}$ value of $1297 \mathrm{~J} / \mathrm{m}$ for a hydration number calculation, that means the small cage occupancy is nearly 0.3136 assuming the $98 \%$ occupancy for large cage.

Cage occupancy can be calculated at a particular temperature from Equation 3.10 using the Langmuir constant obtained from our carbon dioxide $a b$ initio potentials in Table 3.3. The hydration number can be determined from cage occupancies using Equation 3.19. In Figure 3.10 the predictions for the cage occupancy ratios $\left(\theta_{s} / \theta_{L}\right)$ for the carbon dioxide hydrates obtained by our site-site model and by other researchers are compared. Ripmeester and Ractliff $^{21}$ gave a lower limit to the hydration number of 7.0 for $\mathrm{CO}_{2}$ hydrate, cage occupancy ratios $\left(\theta_{s} / \theta_{L}\right)$ as 0.32 , at temperature $268 \mathrm{~K}$ and pressure of 9.9 bar. This means that the hydration number should be higher than 7.0 and the small cage occupancy should be in the range of 25 to $40 \%$. CSMGEM a thermodynamic code developed by Sloan ${ }^{1}$, Colorado School of Mines, to predict the phase equilibrium of the hydrate and it uses the fitted Kihara potential 
parameters in predicting the occupancies and phase equilibria ${ }^{1}$. The cage occupancy predicted by CSMGEM for small cage is in between $47 \%$ and $40 \%$ in the temperature between $256 \mathrm{~K}$ and 283.3 K and almost fully occupied for large cages, 97\% occupancy for large cage. The Sloan/CSMGEM predicted the phase equilibrium of carbon dioxide hydrate accurately but it over estimates the cage occupancies. Klauda and Sandler ${ }^{9}$ predicted the small cage occupancy in between $54 \%$ and $90 \%$ in the temperature between $243.1 \mathrm{~K}$ and $290 \mathrm{~K}$. Sun and Duan ${ }^{22}$ using the site-site ab initio model had reported the hydration number for only two temperatures, at equilibrium conditions, at $273.1 \mathrm{~K}$ and $274.5 \mathrm{~K}$. We have calculated the small cage occupancy for Sun and Duan data from hydration number assuming 99\% occupancy for large cage and obtained as $55 \%$ and $60 \%$ occupancy at $273.15 \mathrm{~K}$ and $274.5 \mathrm{~K}$.

The cage occupancies predicted by Sloan/CSMGEM, Klauda and Sandler and Sun and Duan over estimate the small cage occupancies. The small cage occupancies predicted by this site-site model for carbon dioxide structure I hydrate is in the range of $25 \%$ to $38 \%$ for temperatures ranging from $155.5 \mathrm{~K}$ to $283.3 \mathrm{~K}$, where as the large cage is more than $98 \%$ occupied. Figure 3.11 compares the hydration number predicted by this model and by other researchers ${ }^{1,9,21,22}$. 


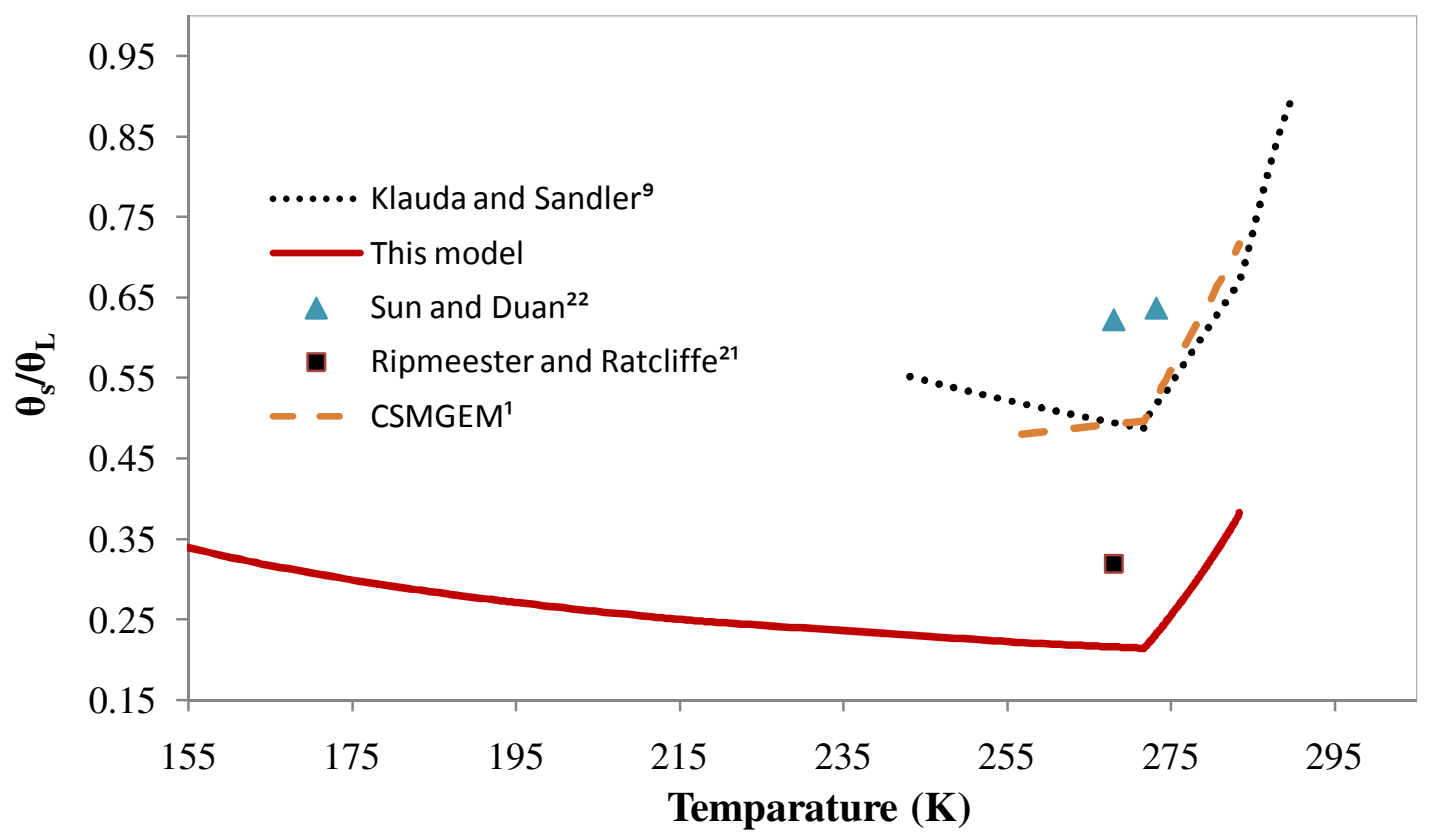

Figure 3.15 Cage occupancy of carbon dioxide hydrate at temperature ranging from $155 \mathrm{~K}$ to $283 \mathrm{~K}$

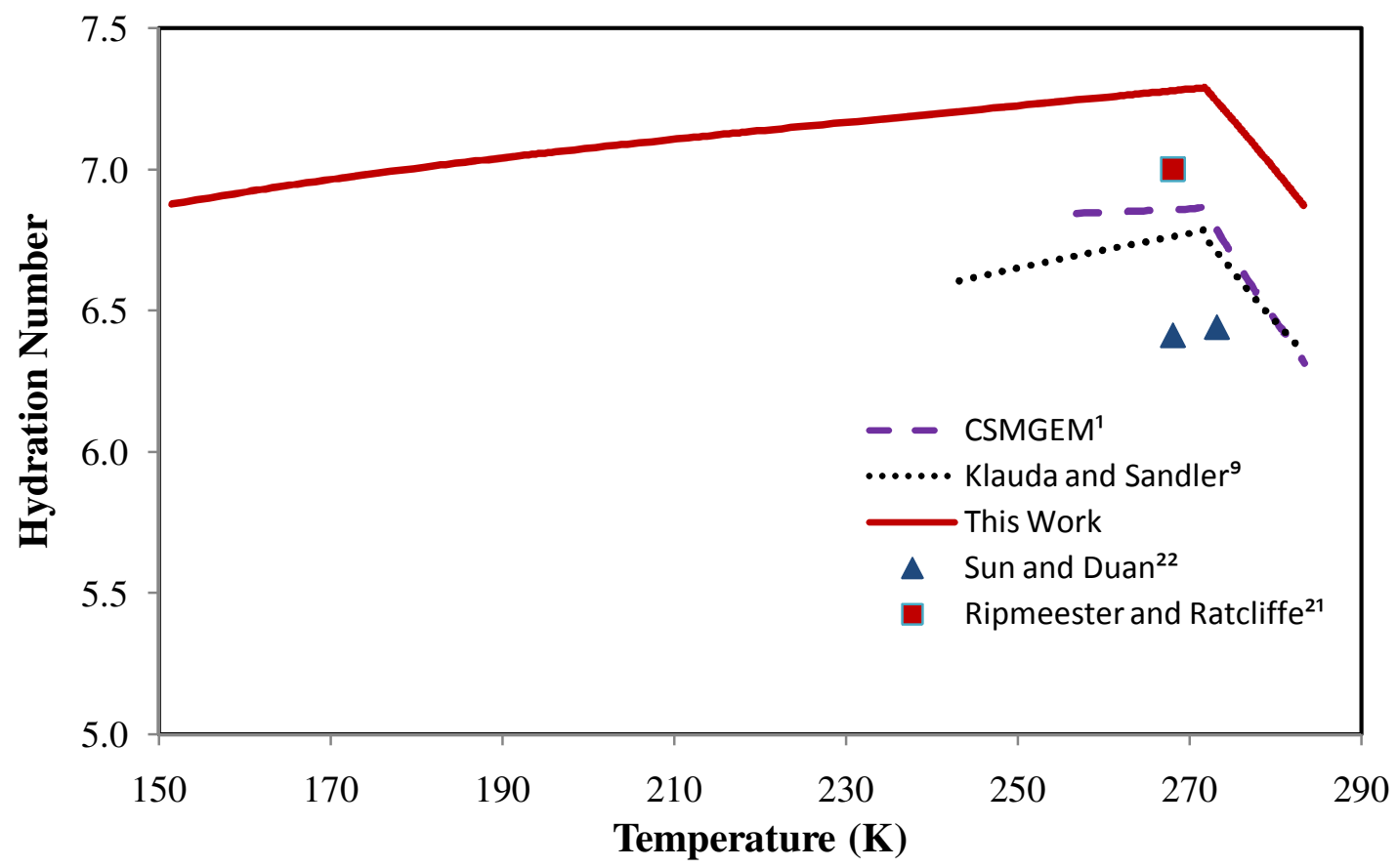

Figure 3.16 Hydration number for carbon dioxide hydrate at different temperature 


\subsection{References}

1. Sloan, E. D.; Koh, C. A. Clathrate hydrates of natural gases, $3^{\text {rd }}$ ed, 2007.

2. Møller, C.; Plesset, M. S. Phys. Rev. 1934, 46, 618.

3. Boys, S.F.; Bernardi, F. Mol.Phys. 1970, 19, 553.

4. Peterson, K. I.; Klemperer, W. J. Chem. Phys. 1984, 80, 2439.

5. Raghavachari, K.; trucks, G.W.; Pople, J.A.; Headgordon, M. A. Chem. Phys. Lett. $1989,157,479$.

6. Dunning, T. H. J. Phys. Chem. A 2000, 104, 9062.

7. Cao, Z. T.; Tester, J. W.; Sparks, K. A.; Trout, B. L. J. Phys. Chem. B. 2001, 105, 10950.

8. Anderson, B. J.; Tester, J. W.; Trout, B. L. J. Phys. Chem. B. 2004, 108, 18705.

9. Klauda, J. B.; Sandler, S. I. J. Phys. Chem. B. 2002, 106, 5722.

10. Holder, G. D.; Malekar, S. T.; Sloan, E.D. Ind. Eng. Chem. Fund. 1984, 23, 123.

11. Dharmavardhana, P. B.; Parrish, W.R.; Sloan, E.D. Ind. Eng. Chem. Fund. 1980, 19, 410.

12. Holder, G. D.; Zetts, S. P.; Pradhan, N. Rev. Chem. Eng. 1988, 5, 1.

13. Pradhan, N. Prediction of Multi-phase Equilibria in Gas Hydrates. 1985, M.S. Thesis, University of Pittsburgh, Pittsburgh, PA.

14. Stackelberg, M. v.; Müller, H.R. Zeitschrift für Electrochemie 1954, 96 , 11022

15. John, V. T.; Papadopoulos, K. D.; Holder, G. D. AIChE J. 1985, 31, 252

16. Holder, G. D.; Corbin, G; Papadopoulos, K. D. Ind. Eng. Chem. Fund. 1980, 19, 282.

17. van der Waals, J.H.; Platteeuw, J.C. Adv. Chem. Phys. 1959, $2,1$.

18. Peng, D.- Y.; Robinson, D. B. Ind. Eng. Chem. Fund. 1976, 15, 59.

19. Henning, R. W.; Schultz. A. J.; Thieu, V.; Halpern, Y. J. Phys.Chem. A 2000, 104, 5066

20. Udachin, K. A.; Ratcliffe, C. I.; Ripmeester, J. A. J. Phys.Chem. B 2001, 105, 4200.

21. Ripmeester, J. A.; Ratcliffe, C. I. Energy Fuels 1998, 12, 197.

22. Sun, R.; Duan, Z. Geochimica et Cosmochimica Acta. 2005, 69, 4411.

23. Harris, G. J.; Yung, H. K. J. Phys. Chem. 1995, 99, 12021.

24. Tester, J. W.; Modell, M. Thermodynamics and its applications, $3^{\text {rd }}$ ed., 1997.

25. Mahoney, M. W.; Jorgensen, W. L. J. Chem. Phys. 2000, 112, 8910.

26. Sum, A. K.; Burruss, R. C.; Sloan, D. E. J. Phys.Chem. B 1997, 101, 7371.

27. Miller, S.L.; Smythe, W.D. Science, 1970, 170, 531.

28. Falabella, B.J. A Study of natural Gas Hydrates, Ph.D. Thesis, University of Massachusetts, University Microfilims, Ann Arbor, 1975

29. Larson, S.D. Phase Studies of the Two-Component Carbon Dioxide-Water system, Involving the Carbon Dioxide Hydrate, University of Illinios, Urbane, IL, 1955.

30. Robinson,D.B.; Mehta, B.R. J.Can.Pet.Tech. 1971, 10, 33.

31. Deaton, W.M, Frost, E.M., Jr. Gas hydrates and Their relation to the Operation of Natural-gas Pipe Lines, U.S. Bureau of Mines Monograph 8, 1946, 101.

32. Ng, H. -J.; Robinson, D. B. Fluid Phase Equilib. 1985, 21, 145.

33. Unruh, C.H., Katz, D.L. Trans. AIME 1949, 186, 83.

34. Adisasmito, S; Frank, R.J.; Sloan, E. D. J. Chem. Eng. Data, 1991, 36, 68

35. Ohgaki, K; Makihara, Y; Takano, K. J. Chem. Eng. Jpn. 1993, $26,558$. 


\section{Application of cell potential method to calculate the phase equilibrium of multi-component system}

\subsection{Introduction}

Even though there is a large database of experimental clathrates phase behavior, theory of clathrates is not well developed and still relies on the ad hoc fitting of experimental data. The empirical constants are fit to experimental data and then used to predict thermodynamic equilibrium conditions. These commonly fitted parameters works very well in the experimental range but fails when extended outside the range of fit and also fails to predict mixed hydrate thermodynamics. Most of the hydrate reservoir simulations have assumed that the hydrate deposit is of pure methane, but there is a great possibility of encountering a complex gas hydrate mixtures. It is also suggested that the carbon dioxide gas can be stored in linkage with methane exploitation, which serve as a sequestration of carbon dioxide and also extraction of methane gas. The present state of mixed hydrate thermodynamics is not well suited to accurately predict an induced carbon dioxide- methane mixed hydrate. The commonly used fitting procedure when used to predict the mixed hydrates thermodynamics, the intermolecular potentials and reference parameters need adjustments to reproduce accurately phase equilibria and structural transitions.

Recently, Anderson et al. ${ }^{1}$ calculated the phase equilibria of multi-component gas hydrate system without fitting to any experimental data. They calculated the phase equilibria of mixed hydrates by using the cell potential method, an application of a novel mathematical

method reported by Bazant and Trout ${ }^{2}$. With this method, they also predicted the structural 
transitions that have been determined experimentally and some structural transitions that have not been examined experimentally.

Bazant and Trout ${ }^{2}$ showed that the temperature dependence of Langmuir constant contains all the necessary information to determine intermolecular potentials. Cell potentials can be directly extract from experimental data by an analytical inversion method based on the standard van der Waals and Platteeuw ${ }^{3}$ statistical model along with the spherical-cell approximation. The resulting potentials are more meaningful and much simpler than those obtained by numerical fitting with Kihara potentials. They calculated the cell potentials for cyclopropane and ethane clathrates hydrates which occupy only one type of cage. Anderson et al. calculated the cell potentials for hydrates for which the Langmuir constants were computed from ab initio data. They found the potential well depths and volumes of negative energy for 16 single component hydrate system. These calculated cell potentials were validated by predicting existing mixed hydrate phase equilibrium data without any fitting parameters and calculated the mixture phase diagrams for methane, ethane, isobutane and cyclopropane mixtures. In this work, similarly, the carbon dioxide-methane mixed hydrate phase equilibria is predicted using the cell potential method.

\subsection{The statistical thermodynamic model}

The basic statistical thermodynamic model for gas hydrates was proposed in 1959 by van der Waals and Platteeuw (vdWP). The van der Waals and Platteeuw model along with a spherical cell model for the interaction potential between the enclathrated guest molecule and the cage of the clathrates hydrate has been used almost entirely to model the phase behavior of hydrate. The chemical potential difference between the hypothetical empty lattice, $\beta$, and fully 
occupied hydrate lattice, $H$, can be expressed as Equation 4.1 by assuming negligible distortions of the empty lattice, single guest occupancy in the cages, and neglecting guest-guest interactions.

$$
\Delta \mu_{w}{ }^{\beta-H}=-k T \sum_{i} v_{i} \ln \left(1+\sum_{j} C_{J i} \hat{f}_{J}\right)
$$

where $v_{i}$ is the number of $i$-types cavities per water molecule, $\hat{f}_{J}$ is the fugacity of guest molecule $J$ in the gas or liquid phase.

For the structure I hydrate, the unit cell has 46 water molecules with 2 small cavities and 6 large cavities. The number of small cavities per water molecule, $v_{1}$, is equal to $1 / 23$, the number of large cages, $v_{2}$, is equal to $3 / 23$, the complete expression for a pure component structure I water clathrates system is

$$
\frac{\Delta \mu_{w}^{\beta-H}}{k T}=\frac{1}{23} \ln \left(1+C_{J 1} \hat{f}_{J}\right)+\frac{3}{23} \ln \left(1+C_{J 2} \hat{f}_{J}\right)
$$

The structure II hydrate unit cell has 136 water molecules with 16 small cavities and 8 large cavities. The ratio of small cavities to water molecules, $v_{1}$, equals $2 / 17$, and the number of large cages, $v_{2}$, is equal to $1 / 17$. The complete expression for a pure component structure II water clathrates system is

$$
\frac{\Delta \mu_{w}^{\beta-H}}{k T}=\frac{2}{17} \ln \left(1+C_{J 1} \hat{f}_{J}\right)+\frac{1}{17} \ln \left(1+C_{J 2} \hat{f}_{J}\right)
$$

The fugacity, $\hat{f}_{J}$, can be calculated from a mixture form of a PVTN Peng-Robinson equation of state. $T$ is the temperature, and $C_{J i}$ is the temperature dependent Langmuir constant for species $J$ in cavity $i$ defined as 


$$
C_{j i}=\frac{Z_{j i}}{k T}=\frac{1}{8 \pi^{2} k T} \int_{V} \exp \left(-\frac{\Phi(r, \theta, \phi, \alpha, \beta, \gamma)}{k T}\right) r^{2} \sin \theta d r d \theta d \phi d \alpha d \beta d \gamma
$$

where $Z_{j i}$ is the configurational integral and $\Phi(r, \theta, \phi, \alpha, \beta, \gamma)$ is the total interaction potential between the guest molecule and the host molecules surrounding it. The $\Phi(r, \theta, \phi, \alpha, \beta, \gamma)$ is the function of general six-dimensional form of the interaction potential between the spherical coordinates, $r, \theta$ and $\phi$, of the guest molecule and the Euler angles, $\alpha, \beta$ and $\gamma$, that describes the orientation of the guest molecule with respect to all of the water molecules in the clathrates hydrate. The interaction potential was approximated by a Lennard-Jones 6-12 potential with two parameters or by a Kihara potential, with three parameters. The Kihara potential because of the three parameters are only empirically superior and yields better results than L J 6-12 potentials. These empirically fitted potentials are not fundamentally based on the guest-host interactions and relay on the ad hoc adjustments of potential parameters to fit the experimental data, which have been shown to be aphysical and do not match those determined from second virial coefficient and viscosity data ${ }^{4-6}$. The carbon dioxide-water intermolecular potentials are computed from ab initio quantum mechanics and are shown in Chapter 3, which seem to provide an independent means to obtain these potentials. With these intermolecular potentials, the chemical phase equilibrium and cage occupancies are predicted. The reference parameters used are found in Figure 3.8.

In the spherical cell approximation, which is analogous to the approximation made by Lennard-Jones Devonshire in the case of liquids ${ }^{8}$, the total interaction potential, $\Phi(r, \theta, \phi, \alpha, \beta, \gamma)$, is replaced by a spherically averaged cell potential $W(r)$. This reduces the multidimensional configurational integral given in Equation 4.2 to one dimensional radial integral, and the Langmuir constant is given as 


$$
C_{J i}=\frac{4 \pi}{k T} \int_{0}^{R} \exp \left(-\frac{W(r)}{k T}\right) r^{2} d r
$$

where the cutoff distance $R$ is taken as the average radius of the cage, the exact value of $R$ is rarely matters because the temperatures at which hydrates form, the high-energy portion of the cage $r \approx R$ makes a negligible contribution to the integral.

\subsection{Configurational Integral Calculation}

The functional form of cell potential, $W(r)$, can be determined from angle averaging analytically and is given as

$$
\left\langle\frac{W(r)}{k T}\right\rangle=\frac{1}{4 \pi} \int_{0}^{2 \pi} \int_{0}^{\pi} \Phi_{j i}(r, \theta, \phi) \sin \theta d \theta d \phi
$$

The inter molecular potential, $\Phi_{j i}$, is represented by Lennard- Jones 6-12 or by Kihara potential form, using the Kihara potential as shown in Equation 2.25 for the guest- host interactions, the spherically averaged cell potential obtained is

$$
\left\langle\frac{W(r)}{k T}\right\rangle=2 z \varepsilon\left[\frac{\sigma^{12}}{R^{11} r}\left(\delta^{10}+\frac{a}{R} \delta^{11}\right)-\frac{\sigma^{6}}{R^{5} r}\left(\delta^{4}+\frac{a}{R} \delta^{5}\right)\right]
$$

where

$$
\delta^{N}=\frac{1}{N}\left[\left(1-\frac{r}{R}-\frac{a}{R}\right)^{-N}-\left(1+\frac{r}{R}-\frac{a}{R}\right)^{-N}\right]
$$

where $N$ is 4, 5, 10, 11 indicated in Equation 4.6, $z$ is the coordination number of the cavity, $R$ is the effective cavity radius, $r$ is the distance of the guest molecule from the cavity center, $a$ is the core radius of interaction, $\sigma$ is the distance between molecular cores at which there is no interaction, and $\varepsilon$ is the depth of the intermolecular potential well. The Kihara parameters are 
generally determined by fitting the monovariant pressure-temperature equilibrium data numerically, but these fitted parameters lacks any physical significance and also they are not unique and several set of parameters can fit the experimental data well.

\subsection{Inversion of Langmuir Curves}

Alternative to the empirical fitting of Kihara potential to experimental data, it would be preferable to extract more reliable functional form of interatomic potentials without any ad hoc assumptions. Bazant and Trout ${ }^{2}$ described a method by which the functional form of intermolecular potentials can be found by solving Equation 4.5 analytically for $W(r)$, given a particular Langmuir cure $C_{J i}(T)$. The Equation 4.5 is restructured, letting $\beta=1 / k T$, as

$$
C_{J i}(\beta)=4 \pi \beta \int_{0}^{R} e^{-\beta W(r)} r^{2} d r
$$

Here the upper limit of integration is extended to $R=\infty$, this introduces the negligible errors due to the very low temperatures accessible in clathrate experiments. A functional form of $C_{J i}(\beta)$ must be found in order to invert the Equation 4.9 and to calculate the $W(r)$. This is found by computing $C_{J i}(\beta)$ from expermental data and from ab initio data and fitting the computed values of $C_{J i}(\beta)$ to a functional form ${ }^{1}$.

\subsubsection{Unique central-well solution}

The functional form for $C_{J i}(\beta)$ is constructed by some straight-forward fitting of Langmuir constant experimental data, and this can be described well by a van't Hoff temperature dependence given as 


$$
C(\beta)=C_{0} e^{m \beta}
$$

where $C_{0}$ and $m$ are constants and are specific to guest molecule $J$ and cavity $i$. Bazant and Trout illustrated the empirical van't Hoff behavior for ethane and cyclopropane clathrate hydrates. Combining Equation 4.9 and Equation 4.10, the integral equation obtained is as

$$
C_{0} e^{m \beta}=4 \pi \beta \int_{0}^{R} e^{-\beta W(r)} r^{2} d r
$$

There are an infinite many number of solutions to the integral, but the unique central-well solution is a well behaved analytic function. All other non-central-well solutions are aphysical, having discontinuities or cusps in the potential. Therefore, the central-well solution is selected to the Equation 4.11 to represent the van't Hoff temperature dependence. Thus

$$
C(\beta)=\beta F(\beta) e^{-\beta W\left(r_{0}\right)}
$$

where

$$
F(\beta)=\beta \int_{0}^{\infty} e^{-\beta y} g(y) d y
$$

where $g(y)$ is the inverse Laplace transform of the function given as:

$$
G(\beta)=\frac{F(\beta)}{\beta}=\frac{C(\beta) e^{\beta w_{0}}}{\beta^{2}}
$$

These lead to the general expression for the central-well potential, $W(r)$, that exactly reproduces any admissible Langmuir curve, it is given as:

$$
W(r)=W_{0}+g^{-1}\left(\frac{4}{3} \pi r^{3}\right)
$$

In the perfect van't Hoff case, $F(\beta)=C_{0} / \beta$ and $G(\beta)=C_{0} / \beta^{2}$. The inverse Laplace transformers of these functions are simply $f(y)=C_{0} H(y)$ and $g(y)=C_{0} y H(y)$, 
respectively, where $H(y)$ is the Heaviside step function. Finally, the solution to the Equation 4.11, the unique central-well solution, is linear in the volume and cubic in radius and is given as

$$
W(r)=\frac{4 \pi r^{3}}{3 C_{0}}-m \quad \text { for } \quad r \geq 0
$$

The Langmuir hydrate constant curves, $C(\beta)$, are well fit by an ideal van't Hoff temperature dependence, demonstrated by

$$
\log C=m \beta+\log C_{0}
$$

and the slope, $m$, of the van't Hoff plot is equal to the well depth $m=-W_{0}$ and the $y$-intercept $\log C_{0}$ is related to the well size measured by the volume of negative energy $m C_{0}$. This volume corresponds to a spherical radius of

$$
r_{S}=\left(\frac{3 m C_{0}}{4 \pi}\right)^{1 / 3}
$$

The cell potential is simplified as

$$
W(r)=m\left[\left(\frac{r}{r_{s}}\right)^{3}-1\right] \quad \text { for } \quad r \geq 0
$$

The unknown values $m$ and $C_{0}$ can be found by calculating the Langmuir constants over a range of temperatures for a given guest molecule $J$ in the hydrate cage.

\subsubsection{Calculation of Langmuir constant}

The Langmuir constant can be directly calculated from the experimental dissociation data for the case where clathrate hydrates contain a single type of guest molecule occupying only one type of cage. Ethane, cyclopropane, isobutene, propane and certain CFC water 
clathrates occupy only the larger cage of the hydrate. For these with single occupancy, the Equation 4.2 and 4.3 reduces to the following:

for structure I

$$
\frac{\Delta \mu_{w}{ }^{\beta-H}}{k T}=\frac{3}{23} \ln \left(1+C_{J 2} \hat{f}_{J}\right)
$$

for structure II

$$
\frac{\Delta \mu_{w}{ }^{\beta-H}}{k T}=\frac{1}{17} \ln \left(1+C_{J 2} \hat{f}_{J}\right)
$$

$\Delta \mu_{w}{ }^{\beta-H}$ is the chemical potential difference between the hypothetical empty hydrate and water in aqueous liquid phase or in ice phase. $\hat{f}_{J}$ is the fugacity calculated for the fluid phase using the PVTN mixture form of the Peng-Robinson equation of state ${ }^{7}$. The experimental Langmuir constants can be obtained by solving Equations 4.20 and 4.21 for $C_{J 1}$ and $C_{J 2}$ and is given as

for structure I

$$
C_{J 2}=\frac{\exp \left(\frac{23}{3} \Delta \mu_{w}^{\beta-L, \alpha}\right)-1}{\hat{f}_{J}}
$$

for structure II

$$
C_{J 2}=\frac{\exp \left(\frac{23}{3} \Delta \mu_{w}^{\beta-L, \alpha}\right)-1}{\hat{f}_{J}}
$$

Langmuir constants can be obtained directly from experimental data for which the larger cage is occupied by the guest molecule using Equations 4.22 and 4.23 for two different structures. For carbon dioxide hydrate, where it occupies both large and small cages the Langmuir constant cannot be directly calculated by the procedure discussed above. A single set 
of monovariant phase equilibrium data cannot be used to determine the two Langmuir constants values in Equation 4.2 for structure I. Langmuir constants calculated using the site-site ab initio intermolecular potentials is such a method ${ }^{1}$. Langmuir constants were calculated at various temperatures by integrating six-dimensional configurational integral, these Langmuir constants are independent of any fitting parameters. With this site-site $a b$ initio method Langmuir constants can also be computed for unstable structure II carbon dioxide hydtare ${ }^{1}$. Carbon dioxide typically form structure I hydrate but it forms structure II hydrate with other guests like nitrogen. Anderson et al. ${ }^{1}$ has calculated Langmuir constant for the cages of theoretical (unstable) structure II methane hydrate with the above method.

\subsection{Computing Cell Potentials}

Anderson et al. ${ }^{1}$ has regressed the Cell potential parameters from van't Hoff plots, Equation for guest molecule that occupy only the large cage, ethane, cyclopropane and chlorodifluoromethane. They also regressed the Cell potential parameters for methane and Argon for structure I and structure II from the Langmuir constants values computed from sitesite ab initio potentials.

Cell potential parameters for carbon dioxide hydrate are regressed by using $95 \%$ confidence intervals and the regressed Cell potential parameters are given in Table 4.1 for structure I and in Table 4.2 for Structure II. Figure 4.1 shows the van't Hoff temperature dependence for structure I carbon dioxide hydrate small and large cages. 


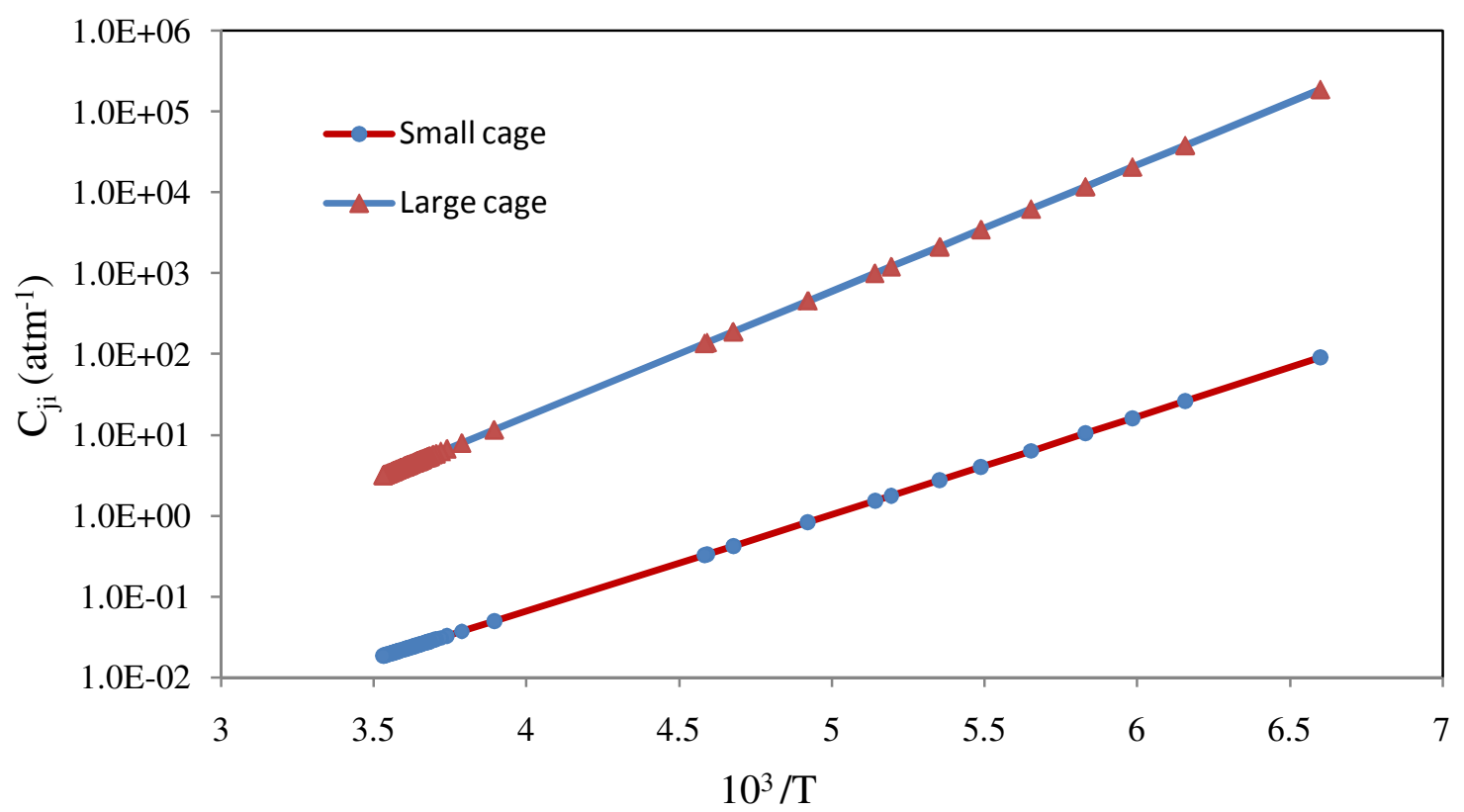

Figure 4.1 van't Hoff behavior indicating the temperature dependency of Langmuir constant.

Table 4.1 Cell potential parameters for structure I carbon dioxide hydrates

\begin{tabular}{|c|c|c|}
\hline Cages & $-w_{0}(\mathrm{kcal} / \mathrm{mol})$ & $r_{s}(\AA)$ \\
\hline Small cage $\left(5^{12}\right)$ & 5.477 & 0.460 \\
\hline Large cage $\left(5^{12} 6^{2}\right)$ & 7.110 & 1.062 \\
\hline
\end{tabular}

Table 4.2 Cell potential parameters for structure II (unstable) carbon dioxide hydrate

\begin{tabular}{|c|c|c|}
\hline Cages & $-w_{0}(\mathrm{kcal} / \mathrm{mol})$ & $r_{s}(\AA)$ \\
\hline Small cage $\left(5^{12}\right)$ & 5.866 & 0.4527 \\
\hline Large cage $\left(5^{12} 6^{2}\right)$ & 6.1407 & 1.9073 \\
\hline
\end{tabular}


The Cell potential parameters were also calculated by above method using Harris and Yung $^{8}$ intermolecular potentials and using Potoff and Siepmann ${ }^{9}$ carbon dioxide and water intermolecular potentials. The intermolecular potentials for carbon dioxide and water system is calculated using the combining rules that is the Lorentz-Berthelot combining rules given in Equation 3.20 and 3.21 and the potentials for water are from TIP4P mode ${ }^{10}$. The Cell potential parameters obtained using their intermolecular potentials are regressed and are given in Table 4.3 and the resulting Cell potentials are shown in Figure 4.2 and 4.3.

The Cell potentials obtained by site-site ab initio potentials for carbon dioxide hydrate are shown in the Figure 4.2 for small cage and in Figure 4.3 for large cage. The central-well solutions by this work shown in Table 4.1 and in Table 4.2 are the simplest potentials that can reproduce the calculated Langmuir constants for structure I and II respectively. The Cell potentials obtained by Kihara potentials by Equations 4.7 and 4.8 are also shown in Figure 4.2 and 4.3 for small and large cages. The Kihara potential parameters are taken from Sloan and $\mathrm{Koh}^{4}$ for carbon dioxide hydrate. The Cell potentials obtained using Harris and Yung ${ }^{8}$ and Potoff and Siepmann ${ }^{9}$ are almost similar, the potential well depth is very less and so they underestimate the cage occupancies for carbon dioxide hydrate. 
Table 4.3 Cell potential parameters for structure I hydrate using other intermolecular potentials

\begin{tabular}{|c|c|c|}
\hline Cages & $-w_{0}(\mathrm{kcal} / \mathrm{mol})$ & $r_{s}(\AA)$ \\
\hline $\begin{array}{c}\text { Using Harris and Yung } \\
\text { Potentials Small cage } \\
\left(5^{12}\right)\end{array}$ & 2.8435 & 0.3573 \\
\hline $\begin{array}{c}\text { Harris and Yung } \\
\text { Potentials, Large cage } \\
\left(5^{12} 6^{2}\right)\end{array}$ & 4.9701 & 0.9618 \\
\hline $\begin{array}{c}\text { Using Pottoff and } \\
\text { Seipmenn potentials } \\
\text { Small cage }\left(5^{12}\right)\end{array}$ & 2.7603 & 0.3481 \\
\hline $\begin{array}{c}\text { Pottoff and Seipmen } \\
\text { potentials, Large cage } \\
\left(5^{12} 6^{2}\right)\end{array}$ & 4.9703 & 0.9499 \\
\hline
\end{tabular}

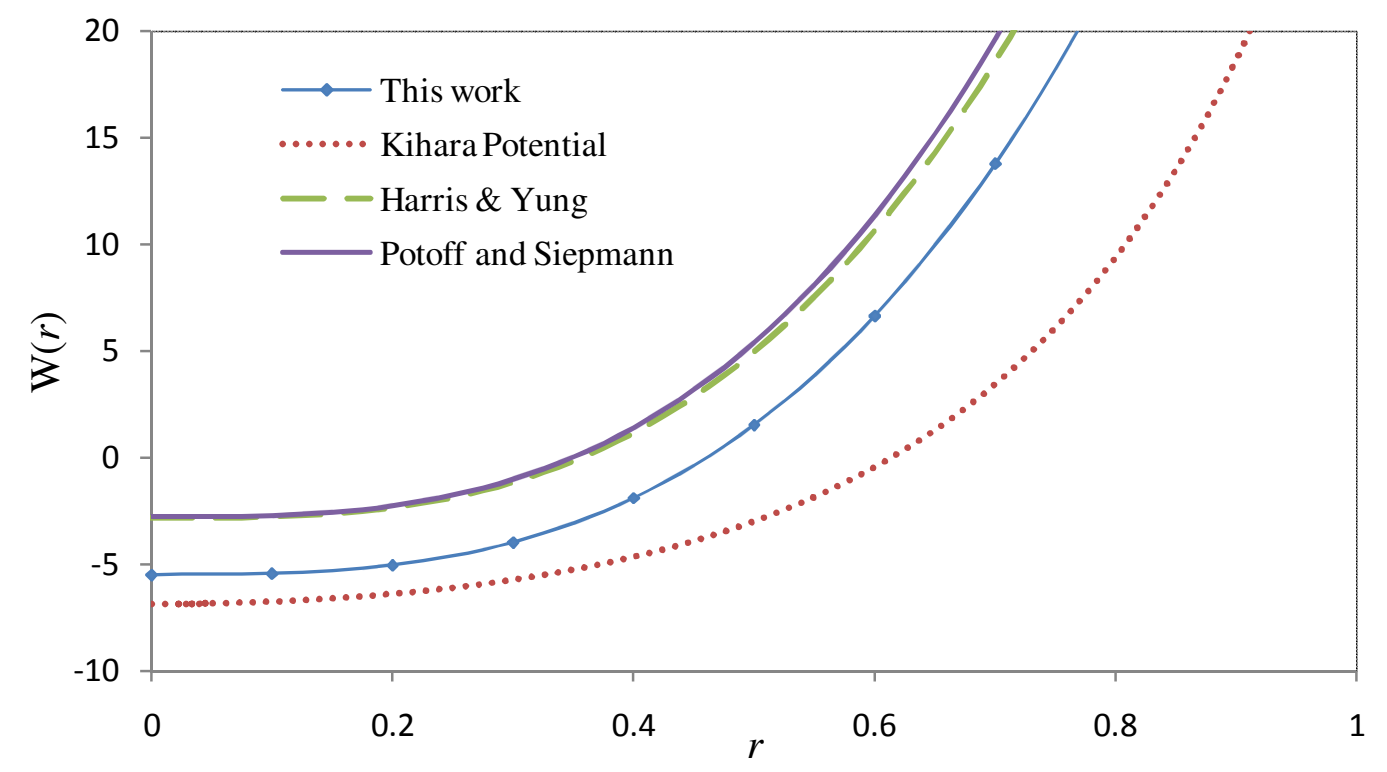

Figure 4.2 Cell potentials of carbon dioxide in small cage structure I hydrate calculated using ab initio site-site potentials 


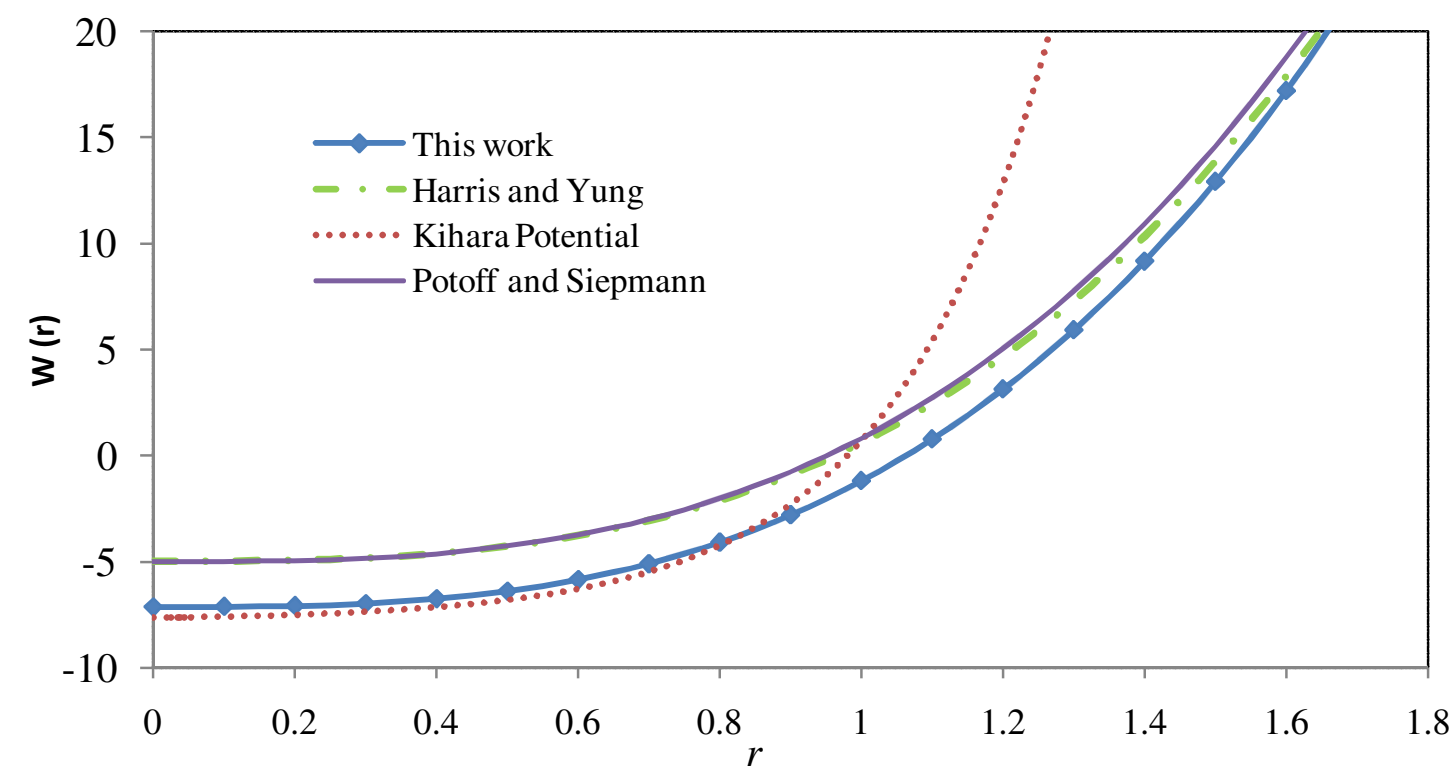

Figure 4.3 Cell potentials of carbon dioxide in large cage structure I hydrate calculated using $a b$ initio site-site potentials 


\subsection{References}

1. Anderson, B. J.; Bazant, M. Z.; Tester, J. W.; Trout, B. L. J. Phys. Chem. B. 2004, 108, 18705.

2. Bazant, Z. M.; Trout, L. B. Physica A 2001, 300, 139.

3. van der Waals, J.H.; Platteeuw, J.C. Adv. Chem. Phys. 1959, 2, 1.

4. Sloan, E. D.; Koh, C. A. Clathrate hydrates of natural gases, $3^{\text {rd }} \mathrm{ed}, 2007$.

5. John, V. T.; Papadopoulos, K. D.; Holder, G. D. AIChE J. 1985, 31, 252

6. John, V. T.; Holder, G. D. J. Phys. Chem. 1985, 89, 3279.

7. Peng, D.- Y.; Robinson, D. B. Ind. Eng. Chem. Fund. 1976, 15, 59.

8. Harris, G. J.; Yung, H. K. J. Phys. Chem. 1995, 99, 12021

9. Potoff, J. J.; Siepmann, I. J. AIChE J. 2001, 47, 1676.

10. Mahoney, M. W.; Jorgensen, W. L. J. Chem. Phys. 2000, 112, 8910. 


\section{Conclusions and Future work}

\subsection{Conclusions}

The overall thesis goal was to better understand the relationship between the microscopic properties and macroscopic properties of the gas hydrate system. An ab initio quantum mechanical calculation has been employed to model the intermolecular potentials between the carbon dioxide-water systems and from which the configurational integral is evaluated. By this ab initio method of evaluating configurational model, a number of specific limitations that were identified by using earlier methods to evaluate the phase equilibrium and cage occupancies has been minimized. With these potentials, macroscopic properties such as thermodynamic phase equilibrium and cage occupancies for carbon dioxide have been calculated accurately. In a more specific way we conclude in this work as:

An $a b$ initio quantum mechanical calculation with MP2/aug-cc-pVTZ basis method has been employed to calculate the intermolecular potentials between the carbon dioxidewater systems. Various methods and basis sets functions has been studied to explore the interaction between the carbon dioxide and water dimer. MP2 method was found to treat the electron correlation well for this dimer compare to more accurate CCSD (T) method and based on the computational cost and accuracy aug-cc-pVTZ basis set is more accurate.

A site-site method has been applied to develop the $\mathrm{CO}_{2}-\mathrm{H}_{2} \mathrm{O}$ intermolecular potentials that characterize the six dimensional potential energy surfaces.

$>$ The $a b$ initio intermolecular potentials obtained from 6,000 point hyperspace energy surface were corrected for many-body effects. The corrections were employed by fitting 
the intermolecular potentials to quantum mechanical calculations on system with 15 water molecules interacting with one carbon dioxide molecule.

The reference thermodynamic parameters were calculated for structure I carbon dioxide hydrate using site-site ab initio potentials as $\Delta \mu_{w}{ }^{o}=1204 \pm 2 \mathrm{~J} / \mathrm{mol}$ and $\Delta H_{w}{ }^{o}=1189$ $\pm 12 \mathrm{~J} / \mathrm{mol}$. The estimation of error in the calculation of reference parameters was found by calculating the $95 \%$ confidence intervals on the regression.

The EPM2 model for carbon dioxide, intermolecular potentials, developed by Harris and Yung has failed to predict the cage occupancies and phase equilibrium when applied to hydrates. The reference parameters obtained by using the Harris and Yung potentials are $\Delta \mu_{w}{ }^{o}=178.4 \pm 3 \mathrm{~J} / \mathrm{mol}$ and $\Delta H_{w}{ }^{o}=95.8 \pm 12 \mathrm{~J} / \mathrm{mol}$ which are nowhere in the range obtained by earlier researchers either numerically or experimentally.

With the site-site ab initio intermolecular potentials and the reference parameters calculated, the phase equilibrium pressure was computed with less than $2 \%$ of absolute average deviation from the experimental data.

$>$ The small cage occupancy predicted by this model for structure $\mathrm{ICO}_{2}$ is in the range of $25 \%$ to $38 \%$ for temperatures ranging from $155.5 \mathrm{~K}$ to $283.3 \mathrm{~K}$, where as the large is more than $98.5 \%$ occupied in the temperature range.

$>$ The cage occupancies predicted by Sloan/CSMGEM, Klauda and Sandler and Sun and Duan over estimated the small cage occupancy compare to the lower limit given for hydration number by Ripmeester and Ratcliff as 7.0. This results in inaccurate potentials used by earlier researchers in predicting the hydrate properties. 
Cell potential parameters are regressed from the Langmuir constants calculated from the site-site $a b$ initio intermolecular potentials. Mixed hydrate properties can be calculated with these cell potential parameters without fitting to any experimental mixture data.

\subsection{Recommendations and Future work}

$>$ The Peng-Robinson equation of state was used in this work to model the fluid fugacity. This EOS works well at the lower pressures, i.e still the second quadruple point, 283.1 $\mathrm{K}$, but fails to accurately model the fluid fugacity at the elevated pressures. Because of this there is much deviation in the predicted pressures after the second quadruple point. There is a need of EOS which can calculate the fugacity of the fluids at higher temperatures, i.e. after second quadruple point.

$>$ In the PES calculation, there are not many points lie on the diagonal for plane 1 and for plane 2 as shown in Figure 3.7 and in Figure 3.8. Therefore a polarizable potential model like the charge on the spring model is needed to improve the optimization of the site-site potentials to the ab initio energies so that lot many points lie on the diagonal.

$>$ The van der Walls and Platteeuw model assumed a non distortion of hydrate lattice, but it has been showed that there is a significant change in the hydrate lattice with the guest molecule. This lattice distortions effect must be incorporated in the model.

$>$ With the regressed Cell potential parameters, carbon dioxide and methane mixed hydrate properties can be calculated which helps in understanding the swapping of methane hydrate with carbon dioxide. 